\title{
A Desingularization of the Moduli Space of Rank 2 Higgs Bundles over a Curve
}

\author{
Sang-Bum Yoo
}

\begin{abstract}
Let $X$ be a smooth complex projective curve of genus $g \geq 3$. Let $\mathbf{M}_{2}$ be the moduli space of semistable rank 2 Higgs bundles with trivial determinant over $X$. We construct a desingularization $\mathbf{S}$ of $\mathbf{M}_{2}$ as a closed subvariety of a moduli space. We prove that $\mathbf{S}$ is a nonsingular variety containing the stable locus of $\mathbf{M}_{2}$ as an open dense subvariety. On the other hand, there is another desingularization $\mathbf{K}$ of $\mathbf{M}_{2}$ obtained from Kirwan's algorithm. We show that $\mathbf{S}$ can be obtained after two blow-downs of $\mathbf{K}$.
\end{abstract}

\section{Introduction}

Recently the birational geometry of various moduli spaces have become one of the main interests in algebraic geometry. It can be applied to construct new examples or to compute various invariants and so forth. On the moduli space of Higgs bundles over an algebraic curve, there have been a few developments in this direction. We can find some of them in $4,7,15$.

Throughout this paper, $X$ denotes a smooth complex projective curve of genus $g \geq 3$.

We first review a construction of a desingularization of the moduli space of vector bundles over an algebraic curve, which was done by C. S. Seshadri. Let $\mathbf{N}$ be the moduli space of semistable rank 2 vector bundles over $X$ with trivial determinant. It is known that $\mathbf{N}$ has singularities along $\mathbf{N} \backslash \mathbf{N}^{s}$ where $\mathbf{N}^{s}$ is the stable locus of $\mathbf{N}$. In [20, 21], a nonsingular variety $\widetilde{\mathbf{N}}$ was constructed together with a canonical morphism $\widetilde{\mathbf{N}} \rightarrow \mathbf{N}$ which is an isomorphism on $\mathbf{N}^{s}$. $\widetilde{\mathbf{N}}$ is indeed a closed subvariety of the moduli space of stable rank 4, degree 0 parabolic vector bundles over $X$ with respect to a point on $X$ with sufficiently small weights.

Seshadri's approach mentioned above can be applied to the moduli space of Higgs bundles over an algebraic curve. Let $\mathbf{M}_{2}$ be the moduli space of semistable rank 2 Higgs bundles over $X$ with trivial determinant. It is also known that $\mathbf{M}_{2}$ has singularities along $\mathbf{M}_{2} \backslash \mathbf{M}_{2}^{s}$ where $\mathbf{M}_{2}^{s}$ is the stable locus of $\mathbf{M}_{2}$ (see 13,22 for the details). We construct a desingularization $\mathbf{S}$ of $\mathbf{M}_{2}$. Let $\mathbf{M}_{4,\left(a_{1}, a_{2}\right)}^{\mathrm{par}}$ be the moduli space of semistable

Received May 28, 2019; Accepted September 2, 2020.

Communicated by Yoichi Miyaoka.

2010 Mathematics Subject Classification. 14D15, 14D22, 14E05, 14E15.

Key words and phrases. desingularization, Higgs bundle, parabolic Higgs bundle, specialization of $M(2)$. 
rank 4, degree 0 parabolic Higgs bundles over $X$ with multiplicities $(1,3)$ and weights $\left(a_{1}, a_{2}\right)$ with respect to a point on $X$. We choose sufficiently small weights $\left(a_{1}, a_{2}\right)$ so that $\mathbf{M}_{4,\left(a_{1}, a_{2}\right)}^{\mathrm{par}}=\mathbf{M}_{4,\left(a_{1}, a_{2}\right)}^{\mathrm{par}, s}$ where $\mathbf{M}_{4,\left(a_{1}, a_{2}\right)}^{\mathrm{par}, s}$ is the stable locus of $\mathbf{M}_{4,\left(a_{1}, a_{2}\right)}^{\mathrm{par}}$ (Proposition 3.1). $\mathbf{S}$ is defined as a closed subvariety of $\mathbf{M}_{4,\left(a_{1}, a_{2}\right)}^{\text {par }}$. Precisely, $\mathbf{S}$ is the Zariski closure of $\mathbf{S}^{\prime}$ in $\mathbf{M}_{4,\left(a_{1}, a_{2}\right)}^{\mathrm{par}, s}$, where

$$
\mathbf{S}^{\prime}=\left\{[(E, \phi, s)] \in \mathbf{M}_{4,\left(a_{1}, a_{2}\right)}^{\mathrm{par},} \mid \operatorname{End}((E, \phi)) \cong M(2), \operatorname{det} E \cong \mathcal{O}_{X} \text { and } \phi \text { is traceless }\right\} .
$$

Here $M(2)$ is the $\mathbb{C}$-algebra of $2 \times 2$ matrices with entries in $\mathbb{C}$. We have the following result.

Theorem 1.1. (Theorem 5.9 $\mathbf{S}$ is nonsingular. Furthermore, there is a canonical morphism $\pi_{\mathbf{S}}: \mathbf{S} \rightarrow \mathbf{M}_{2}$ which is an isomorphism on $\mathbf{M}_{2}^{s}$.

For the proof of the first statement, we first show that there exists a bijection between $\mathbf{M}_{2}^{s}$ and $\mathbf{S}^{\prime}$ (Theorem 3.7). Next, we define a moduli functor par-Higgs ${ }_{4,\left(a_{1}, a_{2}\right)}^{s p}$ of $\mathbf{S}$, which is the subfunctor of the moduli functor par-Higgs ${ }_{4,\left(a_{1}, a_{2}\right)}$ of $\mathbf{M}_{4,\left(a_{1}, a_{2}\right)}^{\mathrm{par}}$ parametrizing all families of stable parabolic Higgs bundles equipped with specializations of $M(2)$ on the endomorphism algebras of the underlying Higgs bundles. Roughly speaking, the specialization of $M(2)$ is a limit structure of $\mathbb{C}$-algebra structures of $M(2)$ (see $\$ 2.4$ for the details). Then we show that par-Higgs ${ }_{4,\left(a_{1}, a_{2}\right)}^{s p}$ is formally smooth (Corollary 5.6). To see this, we consider the morphism of functors $\Phi:$ par-Higgs ${ }_{4,\left(a_{1}, a_{2}\right)}^{s p} \rightarrow \mathfrak{S}_{2}$ which maps a family of stable parabolic Higgs bundles to the family of endomorphism algebras of the underlying Higgs bundles, where $\mathfrak{S}_{2}$ is the functor parametrizing all families of specializations of $M(2)$. We show that $\Phi$ is formally smooth (Proposition 5.5). Finally we see that $\mathbf{S}$ is a closed subvariety of $\mathbf{M}_{4,\left(a_{1}, a_{2}\right)}^{\mathrm{par}}$ and is a fine moduli scheme of par-Higgs $\mathrm{s}_{4,\left(a_{1}, a_{2}\right)}^{s p}$ (Proposition 5.8). Then we conclude that $\mathbf{S}$ is nonsingular.

The proof of the second statement is contained in that of Theorem 5.9

There is an interesting application of Theorem 1.1 (Theorem 5.9). Let $\mathbf{K}$ be Kirwan's desingularization of $\mathbf{M}_{2}$ (see $\$ 4$ in this paper, 13,14 for the details). Following O'Grady's argument in [18, 19], a nonsingular variety $\mathbf{K}_{\epsilon}$ can be obtained after two blow-downs of $\mathbf{K}$. But there is no known information on the moduli theoretic meaning of $\mathbf{K}_{\epsilon}$. The first author of [12] asked how we can provide the moduli theoretic meaning of $\mathbf{K}_{\epsilon}$. We prove the following result.

Theorem 1.2. (Theorem 6.1) $\mathbf{K}_{\epsilon} \cong \mathbf{S}$.

A similar work was already successfully completed on $\mathbf{N}$ in 12 .

Here is an outline of this paper. In $\$ 2$, we recall basic facts about Higgs bundles, parabolic Higgs bundles, specialization of $M(r)$ and upper semicontinuity for the complex 
of flat families of coherent sheaves. In $\$ 3$, we show that there exists a bijective correspondence between stable Higgs bundles of rank $r$ and stable parabolic Higgs bundles of rank $r^{2}$ with an endomorphism algebra of the underlying Higgs bundles as a specialization of $M(r)$. In \$4 we introduce the Kirwan's desingularization of $\mathbf{M}_{2}$. In \$5, we construct $\mathbf{S}$ and then prove Theorem 1.1. In $\$ 6$, we state Theorem 1.2 precisely. In $\$ 7$, we prove Theorem 1.2 .

\section{Preliminaries}

In this section, we recall some basic facts which are useful throughout this paper.

\subsection{Higgs bundles}

Let $r$ be an integer with $r \geq 2$. A Higgs bundle of rank $r$ is a pair of a rank $r$ vector bundle $E$ with trivial determinant and a section $\phi$ of $H^{0}\left(X, \mathcal{E} n d_{0} E \otimes K_{X}\right)$, where $K_{X}$ is the canonical bundle of $X$ and $\mathcal{E} n d_{0} E$ denotes the traceless part of $\mathcal{E} n d E$. To construct the moduli space of Higgs bundles, a stability condition has to be imposed. C. T. Simpson introduced the following (see [22]).

Definition 2.1. (1) A Higgs bundle $(E, \phi)$ is semistable (resp. stable) if for any nonzero proper subbundle $F$ satisfying $\phi(F) \subset F \otimes K_{X}$, we have

$$
\mu(F):=\frac{\operatorname{deg} F}{\operatorname{rank} F} \leq 0 \quad\left(\operatorname{resp} . \mu(F):=\frac{\operatorname{deg} F}{\operatorname{rank} F}<0\right) .
$$

(2) A Higgs bundle $(E, \phi)$ is polystable if it is either stable or a direct sum of stable Higgs bundles.

A morphism of Higgs bundles from $(E, \phi)$ to $(F, \psi)$ is a morphism of vector bundles $g: E \rightarrow F$ such that $\psi g=\left(g \otimes \operatorname{id}_{K_{X}}\right) \phi$, denoted by $g:(E, \phi) \rightarrow(F, \psi)$. Throughout this paper, the space of morphisms of Higgs bundles from $(E, \phi)$ to $(F, \psi)$ is denoted by $\operatorname{Hom}((E, \phi),(F, \psi))$. In particular, $\operatorname{Hom}((E, \phi),(E, \phi))$ is denoted by $\operatorname{End}((E, \phi))$. We call $(F, \psi)$ a Higgs subbundle of $(E, \phi)$ if there is an injective morphism of Higgs bundles $(F, \psi) \hookrightarrow(E, \phi)$.

Let $S$ be a scheme of finite type over $\mathbb{C}$. Let $p_{1}: X \times S \rightarrow X$ and $p_{2}: X \times S \rightarrow S$ be the projections onto the first and the second components respectively. A pair $(\mathcal{E}, \varphi)$ of a vector bundle $\mathcal{E}$ on $X \times S$ and a morphism $\varphi: \mathcal{E} \rightarrow \mathcal{E} \otimes p_{1}^{*} K_{X}$ of vector bundles is $a$ family of (semi)stable Higgs bundles of rank $r$ on $X \times S$ if the restrictions $\left(\mathcal{E}_{s}, \varphi_{s}\right)$ to the geometric fibers $X \times\{s\}$ are (semi)stable Higgs bundles of rank $r$ on $X$.

Let $(\mathcal{E}, \varphi)$ be a family of (semi)stable Higgs bundles of rank $r$ on $X \times S$ and let $\left\{\left(U_{i}, z_{i}\right)\right\}$ be a pair of an open cover and local coordinate of $X$ such that $\left.\mathcal{E}\right|_{U_{i}} \cong \mathcal{O}_{U_{i} \times S}^{\oplus r}$ 
and $\left.\varphi\right|_{U_{i}}=A_{i} d z_{i}$ where $A_{i}$ is an $r \times r$ matrix with entries in $H^{0}\left(U_{i} \times S, \mathcal{O}_{U_{i} \times S}\right)$. We call $\left\{\left.\varphi\right|_{U_{i}}\right\}$ (or $\left.\left\{A_{i}\right\}\right)$ a local Higgs field of $(\mathcal{E}, \varphi)$ with respect to $\left\{\left(U_{i}, z_{i}\right)\right\}$.

The set of isomorphism classes of polystable Higgs bundles of rank $r$ with trivial determinant admits a structure of irreducible normal quasi-projective variety of dimension $\left(r^{2}-1\right)(2 g-2)$ (see 10,17,22]) and we denote it by $\mathbf{M}_{r}$. The locus $\mathbf{M}_{r}^{s}$ of stable Higgs bundles in $\mathbf{M}_{r}$ is a smooth open dense subvariety of $\mathbf{M}_{r}$.

By [22], any Higgs bundle can be identified with a coherent sheaf on the total space of the canonical bundle $\left|K_{X}\right|=\operatorname{Spec}\left(S^{\bullet} K_{X}^{\vee}\right)$. Let $Z=\operatorname{Proj}\left(S^{\bullet}\left(K_{X}^{\vee} \oplus \mathcal{O}_{X}\right)\right)$ and let $D=Z-\left|K_{X}\right|$ be the divisor at infinity. The projection $\left|K_{X}\right| \rightarrow X$ extends to a map $\pi: Z \rightarrow X$. Choose a positive integer $k$ so that $\mathcal{O}_{Z}(1):=\pi^{*} \mathcal{O}_{X}(k) \otimes_{\mathcal{O}_{Z}} \mathcal{O}_{Z}(D)$ is ample on $Z$. In particular, $\mathcal{O}_{\left|K_{X}\right|}(1)=\pi^{*} \mathcal{O}_{X}(k)$. Thus for any coherent sheaf $\mathcal{E}$ on $Z$ such that $\operatorname{Supp}(\mathcal{E}) \cap D=\emptyset, \chi(\mathcal{E}(m))=\chi\left(\left(\pi_{*} \mathcal{E}\right)(k m)\right)$ by the projection formula and the fact that $\left.\pi\right|_{\left|K_{X}\right|}$ is affine. Furthermore, by virtue of [2], we can describe $\operatorname{Supp}(\mathcal{E})$ explicitly as a spectral curve.

Theorem 2.2. (see [2, Proposition 3.6], [8, Proposition 6.1] and [22, Lemma 6.8])

(1) A Higgs bundle $(E, \phi)$ on $X$ is the same thing as a coherent sheaf $\mathcal{E}$ on $Z$ such that $\pi_{*} \mathcal{E}=E$ and $\operatorname{Supp}(\mathcal{E}) \cap D=\emptyset$. This identification gives an equivalence of categories.

(2) The notions of semistability and stability for a Higgs bundle $(E, \phi)$ on $X$ are the same as the corresponding notions for the coherent sheaf $\mathcal{E}$ on $Z$ associated to $(E, \phi)$ in (1).

(3) Any coherent sheaf $\mathcal{E}$ on $Z$ associated to $(E, \phi)$ is of pure dimension 1.

(4) For $s=\left(s_{i}\right) \in \bigoplus_{i=2}^{r} H^{0}\left(X, K_{X}^{i}\right)$, there is a bijective correspondence between isomorphism classes of torsion free sheaves $\mathcal{E}$ on $X_{s}$ of rank 1 and isomorphism classes of Higgs bundles $(E, \phi)$ of rank $r$ with coefficients $s_{i}$ of the characteristic polynomial of $\phi$ where the correspondence is given by (1) and the spectral curve $X_{s}$ is defined by

$$
x^{r}+s_{2} x^{r-2}+s_{3} x^{r-3}+\cdots+s_{r}=0
$$

for $x$ the tautological section of $\pi^{*} K_{X}$.

The following fact will be used later in $\$ 3$.

Lemma 2.3. Let $\mathcal{E}$ be the coherent sheaf of pure dimension 1 on $Z$ corresponding to $(E, \phi)$ such that $\operatorname{Supp}(\mathcal{E}) \cap D=\emptyset$. Then

$$
\pi_{*}\left(\iota_{f, *}\left(\left.\mathcal{E}\right|_{f}\right)\right)=\iota_{x_{0}, *}\left(\left.E\right|_{x_{0}}\right)
$$

where $x_{0}$ is a point of $X, \iota_{x_{0}}:\left\{x_{0}\right\} \hookrightarrow X$ is the inclusion of $\left\{x_{0}\right\}$ into $X, f=\pi^{-1}\left(x_{0}\right) \backslash D$ and $\iota_{f}: f \hookrightarrow\left|K_{X}\right|$ is the inclusion of $f$ into $\left|K_{X}\right|$. Furthermore $\operatorname{Supp}\left(\iota_{f, *}\left(\left.\mathcal{E}\right|_{f}\right)\right)$ is a zero-dimensional subscheme of length $\operatorname{rank}(E)$ in $\left|K_{X}\right|$. 
Proof. We may assume that $X=\operatorname{Spec}(A),\left|K_{X}\right|=\operatorname{Spec}(B)$ and $\pi: \operatorname{Spec}(B) \rightarrow \operatorname{Spec}(A)$. Let $M$ be $A$-module and $B$-module, that is, $\widetilde{M}$ is $\widetilde{A}$-module and $\pi_{*} \widetilde{B}$-module. Let $\mathfrak{p}$ be a prime ideal of $A$ corresponding to $x_{0}$. On $\operatorname{Spec}(A)$

$$
\left.\iota_{x_{0}, *} \widetilde{M}^{A}\right|_{x_{0}}=M \otimes \widetilde{\left(_{\left(A_{\mathfrak{p}}\right.} / \mathfrak{p} A_{\mathfrak{p}}\right)^{A}}
$$

and on $\operatorname{Spec}(B)$

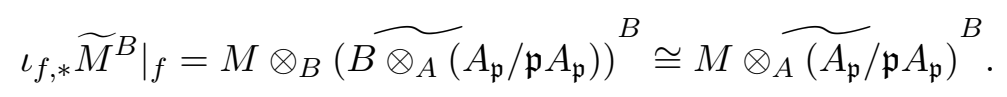

This completes the proof of the first statement.

From Theorem 2.2 (4), $\operatorname{Supp}(\mathcal{E})$ is the spectral curve $X_{s}$ given by a polynomial of degree $\operatorname{rank}(E)$ and $\mathcal{E}$ is a torsion free $\operatorname{sheaf}$ on $\operatorname{Supp}(\mathcal{E})$ of $\operatorname{rank} 1$. Since

$$
-c_{2}\left(\iota_{f, *}\left(\left.\mathcal{E}\right|_{f}\right)\right)=\chi\left(\iota_{f, *}\left(\left.\mathcal{E}\right|_{f}\right)(m)\right)=\chi\left(\iota_{x_{0}, *}\left(\left.E\right|_{x_{0}}\right)(k m)\right)=c_{1}\left(\iota_{x_{0}, *}\left(\left.E\right|_{x_{0}}\right)\right)=\operatorname{rank}(E),
$$

we see that $\operatorname{dim} \operatorname{Supp}\left(\iota_{f, *}\left(\left.\mathcal{E}\right|_{f}\right)\right)=0$ and $\operatorname{length}\left(\operatorname{Supp}\left(\iota_{f, *}\left(\left.\mathcal{E}\right|_{f}\right)\right)\right)=\operatorname{rank}(E)$. This completes the proof of the second statement.

\subsection{Extensions of Higgs bundles}

For Higgs bundles $(E, \phi)$ and $\left(E^{\prime}, \phi^{\prime}\right)$, let $\operatorname{Hom}\left(\left(E^{\prime}, \phi^{\prime}\right),(E, \phi)\right)$ be the complex

$$
\mathcal{H o m}\left(E^{\prime}, E\right) \longrightarrow \mathcal{H o m}\left(E^{\prime}, E\right) \otimes K_{X}
$$

given by $\psi \mapsto\left(\psi \otimes \operatorname{id}_{K_{X}}\right) \phi^{\prime}-\phi \psi$. Then we have the following.

Theorem 2.4. 9, (3.2)] $\operatorname{Hom}\left(\left(E^{\prime}, \phi^{\prime}\right),(E, \phi)\right)=\mathbf{H}^{0} \mathbf{H o m}\left(\left(E^{\prime}, \phi^{\prime}\right),(E, \phi)\right)$. The space of extensions of $\left(E^{\prime}, \phi^{\prime}\right)$ by $(E, \phi)$ is $\mathbf{H}^{1} \mathbf{H o m}\left(\left(E^{\prime}, \phi^{\prime}\right),(E, \phi)\right)$.

Here an extension of $\left(E^{\prime}, \phi^{\prime}\right)$ by $(E, \phi)$ is a Higgs bundle $\left(E^{\prime \prime}, \phi^{\prime \prime}\right)$ which fits into the following commutative diagram

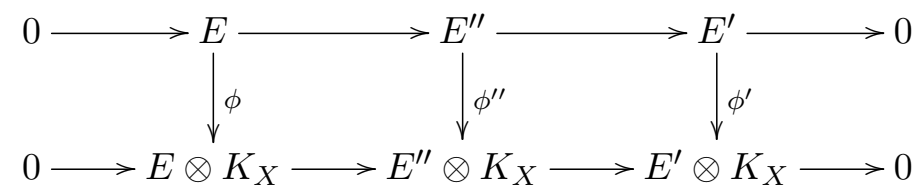

where each row is a short exact sequence. 


\subsection{Parabolic Higgs bundles}

Let $r, n$ be integers with $r \geq 2$ and $n \geq 2$. Let $x_{0}$ be a point of $X$. A parabolic bundle of rank $n$ and degree 0 on $X$, denoted $E_{*}$, with multiplicities $\left(m_{1}, m_{2}, \ldots, m_{l}\right)$ and weights $\left(a_{1}, a_{2}, \ldots, a_{l}\right)$ is a vector bundle $E$ of rank $n$ and degree 0 on $X$ together with a filtration

$$
\left.E\right|_{x_{0}}=F_{1}(E) \supset F_{2}(E) \supset \cdots \supset F_{l}(E) \supset F_{l+1}(E)=\{0\},
$$

weights

$$
0 \leq a_{1}<a_{2}<\cdots<a_{l}<1
$$

and multiplicities

$$
m_{i}=\operatorname{dim}_{\mathbb{C}}\left(F_{i}(E) / F_{i+1}(E)\right) .
$$

Then $\operatorname{pardeg}\left(E_{*}\right):=\operatorname{deg}(E)+\sum_{i=1}^{l} m_{i} \cdot a_{i}$ is called the parabolic degree of $E_{*}$. Denote $\operatorname{par} \mu\left(E_{*}\right):=\operatorname{pardeg}\left(E_{*}\right) / \operatorname{rank}(E)$.

Let $E_{*}$ and $E_{*}^{\prime}$ be parabolic bundles on $X$ with weights

$$
0 \leq a_{1}(E)<a_{2}(E)<\cdots<a_{l}(E)<1 \quad \text { and } \quad 0 \leq a_{1}\left(E^{\prime}\right)<a_{2}\left(E^{\prime}\right)<\cdots<a_{l}\left(E^{\prime}\right)<1
$$

respectively. A bundle morphism $f: E \rightarrow E^{\prime}$ is called strongly parabolic (resp. parabolic) if $f\left(F_{i}(E)\right) \subset F_{j+1}\left(E^{\prime}\right)$ whenever $a_{i}(E) \geq a_{j}\left(E^{\prime}\right)$ (resp. $a_{i}(E)>a_{j}\left(E^{\prime}\right)$ ). The sheaves of parabolic morphisms and strongly parabolic morphisms are denoted by $\mathcal{P a r H o m}\left(E_{*}, E_{*}^{\prime}\right)$ and $\mathcal{S P a r \mathcal { H } o m}\left(E_{*}, E_{*}^{\prime}\right)$ respectively. The spaces of their global sections are denoted by $\operatorname{ParHom}\left(E_{*}, E_{*}^{\prime}\right)$ and $\operatorname{SParHom}\left(E_{*}, E_{*}^{\prime}\right)$.

If $E_{*}$ is a parabolic bundle on $X$ with a filtration

$$
\left.E\right|_{x_{0}}=F_{1}(E) \supset F_{2}(E) \supset \cdots \supset F_{l}(E) \supset F_{l+1}(E)=\{0\}
$$

and weights $\left(a_{1}, a_{2}, \ldots, a_{l}\right)$ and $F$ is a subbundle of $E$, then a parabolic subbundle $F_{*}$ of $E_{*}$ is given by the filtration

$$
\left.F\right|_{x_{0}}=F_{1}(F) \supset F_{2}(F) \supset \cdots \supset F_{l}(F) \supset F_{l+1}(F)=\{0\}
$$

such that $F_{i}(F)=\left.F\right|_{x_{0}} \cap F_{i}(E)$, and the weights

$$
\left(b_{1}, b_{2}, \ldots, b_{l}\right)
$$

such that $b_{j}=a_{i}$ for the largest index $i$ satisfying $F_{j}(F)=\left.F\right|_{x_{0}} \cap F_{i}(E)$.

Definition 2.5. A parabolic Higgs bundle $\left(E_{*}, \phi\right)$ of rank $n$ and degree 0 is a pair of a parabolic bundle $E_{*}$ of rank $n$ and degree 0 , and $\phi \in \operatorname{ParHom}\left(E_{*},\left(E \otimes K_{X}\right)_{*}\right)$ satisfying that $\left(E \otimes K_{X}\right)_{*}$ is a parabolic bundle of rank $n$ and degree 0 such that $F_{i}\left(E \otimes K_{X}\right)=F_{i}(E)$ with the same weights as $E_{*}$. 
Definition 2.6. A parabolic Higgs bundle $\left(E_{*}, \phi\right)$ is said to be semistable (resp. stable) if for every proper parabolic subbundle $F_{*}$ of $E_{*}$ satisfying $\phi\left(F_{*}\right) \subset\left(F \otimes K_{X}\right)_{*}$, we have $\operatorname{par} \mu\left(F_{*}\right) \leq \operatorname{par} \mu\left(E_{*}\right)$ (resp. par $\left.\mu\left(F_{*}\right)<\operatorname{par} \mu\left(E_{*}\right)\right)$.

Let $S$ be a scheme of finite type over $\mathbb{C}$. From now on, assume that $l=2$ and $\left(m_{1}, m_{2}\right)=(1, n-1)$. We write $\left(E_{*}, \phi\right)$ as $(E, \phi, s)$ where $F_{2}(E)$ is the hyperplane defined by $s \in \mathbb{P}\left(\left.E\right|_{x_{0}} ^{\vee}\right)$. If $F_{*}$ is a parabolic subbundle of $E_{*}$, then we write $F_{*}$ as $\left(F, s_{F}\right)$ where $s_{F}=s$ for $\left.F\right|_{x_{0}} \not \subset F_{2}(E)$ and $s_{F}=0$ for $\left.F\right|_{x_{0}} \subset F_{2}(E)$. A triple $(\mathcal{E}, \varphi, \sigma)$ of a vector bundle $\mathcal{E}$ on $X \times S$, a morphism $\varphi: \mathcal{E} \rightarrow \mathcal{E} \otimes p_{1}^{*} K_{X}$ of vector bundles and a section $\sigma \in \mathbb{P}\left(\left.\mathcal{E}\right|_{\left\{x_{0}\right\} \times S} ^{\vee}\right)$ is a family of (semi)stable parabolic Higgs bundles of rank $n$ and degree 0 on $X \times S$ if $\mathcal{E}$ is flat over $S,\left.\mathcal{E}\right|_{\left\{x_{0}\right\} \times S}$ has a filtration

$$
\left.\mathcal{E}\right|_{\left\{x_{0}\right\} \times S}=F_{1}(\mathcal{E}) \supset F_{2}(\mathcal{E}) \supset F_{3}(\mathcal{E})=0
$$

in which $F_{2}(\mathcal{E})$ is a vanishing locus of $\sigma$ and $\operatorname{ker}\left(\left.\mathcal{E} \rightarrow \mathcal{E}\right|_{\left\{x_{0}\right\} \times S} / F_{2}(\mathcal{E})\right)$ is flat over $S$, and if the restrictions $\left(\mathcal{E}_{t}, \varphi_{t}, \sigma_{t}\right)$ to the geometric fibers $X \times\{t\}$ are (semi)stable parabolic Higgs bundles of rank $n$ and degree 0 on $X$.

Definition 2.7. For each scheme $S$ of finite type over $\mathbb{C}$, set $\operatorname{par-Higgs}_{n,\left(a_{1}, a_{2}\right)}(S)=\{(\mathcal{E}, \varphi, \sigma) \mid(\mathcal{E}, \varphi, \sigma)$ is a family of semistable parabolic Higgs bundles of rank $n$ and degree 0 on $X \times S$ with the following properties $\} / \sim$

such that

(i) for every geometric point $t \in S$, the restriction $\left(\mathcal{E}_{t}, \varphi_{t}, \sigma_{t}\right)$ is a semistable parabolic Higgs bundle of rank $n$ and degree 0 on $X$ with weights $0 \leq a_{1}<a_{2}<1$ and multiplicities $(1, n-1)$,

(ii) $(\mathcal{E}, \varphi, \sigma) \sim\left(\mathcal{E}^{\prime}, \varphi^{\prime}, \sigma^{\prime}\right)$ if and only if $(\mathcal{E}, \varphi, \sigma) \cong\left(\mathcal{E}^{\prime}, \varphi^{\prime}, \sigma^{\prime}\right) \otimes p_{2}^{*} \mathcal{L}$ for some invertible sheaf $\mathcal{L}$ on $S$.

Here par-Higgs $_{n,\left(a_{1}, a_{2}\right)}$ is a contravariant functor from the category of schemes of finite type over $\mathbb{C}$ to the category of sets. par-Higgs ${ }_{n,\left(a_{1}, a_{2}\right)}$ denotes the subfunctor of par-Higgs $_{n,\left(a_{1}, a_{2}\right)}$ consisting of all families of stable parabolic Higgs bundles.

Theorem 2.8. (see [23, Theorem 4.6 and Corollary 4.7] and [24, Theorem 5.2]) There exists an irreducible normal quasi-projective variety $\mathbf{M}_{n,\left(a_{1}, a_{2}\right)}^{\mathrm{par}}$ over $\mathbb{C}$ such that $\mathbf{M}_{n,\left(a_{1}, a_{2}\right)}^{\mathrm{par}}$ is a coarse moduli scheme of par-Higgs ${ }_{n,\left(a_{1}, a_{2}\right)}$. 
For parabolic Higgs bundles $(E, \phi, s)$ and $\left(E^{\prime}, \phi^{\prime}, s^{\prime}\right)$, let $\operatorname{Hom}\left((E, \phi, s),\left(E^{\prime}, \phi^{\prime}, s^{\prime}\right)\right)$ be the complex

$$
\mathcal{P a r H o m}\left((E, s),\left(E^{\prime}, s^{\prime}\right)\right) \longrightarrow \mathcal{S P a r H o m}\left((E, s),\left(E^{\prime}, s^{\prime}\right)\right) \otimes K_{X}
$$

given by $\psi \mapsto\left(\psi \otimes \operatorname{id}_{K_{X}}\right) \phi-\phi^{\prime} \psi$. Then we have the following result.

Theorem 2.9. [3] There is a long exact sequence

$$
\begin{aligned}
0 & \rightarrow \mathbf{H}^{0}\left(\mathbf{H o m}\left((E, \phi, s),\left(E^{\prime}, \phi^{\prime}, s^{\prime}\right)\right)\right) \rightarrow H^{0}\left(\mathcal{P} \operatorname{ar} \mathcal{H o m}\left((E, s),\left(E^{\prime}, s^{\prime}\right)\right)\right) \\
& \rightarrow H^{0}\left(\mathcal{S P a r H o m}\left((E, s),\left(E^{\prime}, s^{\prime}\right)\right) \otimes K\right) \rightarrow \mathbf{H}^{1}\left(\operatorname{Hom}\left((E, \phi, s),\left(E^{\prime}, \phi^{\prime}, s^{\prime}\right)\right)\right) \\
& \rightarrow H^{1}\left(\mathcal{P} \operatorname{Pr} \mathcal{H o m}\left((E, s),\left(E^{\prime}, s^{\prime}\right)\right) \rightarrow H^{1}\left(\mathcal{S P a r} \mathcal{H o m}\left((E, s),\left(E^{\prime}, s^{\prime}\right)\right) \otimes K\right)\right. \\
& \rightarrow \mathbf{H}^{2}\left(\operatorname{Hom}\left((E, \phi, s),\left(E^{\prime}, \phi^{\prime}, s^{\prime}\right)\right)\right) \rightarrow 0 .
\end{aligned}
$$

\subsection{The set of specialization of $M(r)$}

This subsection will be useful in $\S 4$ and $\S 5$. Let $M(r)$ be the $\mathbb{C}$-algebra of $r \times r$ matrices with entries in $\mathbb{C}$. Fix a nonzero element $e_{0} \in \mathbb{C}^{r^{2}}$. Let $\mathcal{A}(r)$ be the set of elements in $\operatorname{Hom}\left(\mathbb{C}^{r^{2}} \otimes \mathbb{C}^{r^{2}}, \mathbb{C}^{r^{2}}\right)$ which gives us an algebra structure on $\mathbb{C}^{r^{2}}$ with the identity element $e_{0}$. There is a subset of $\mathcal{A}(r)$ which consists of algebra structures on $\mathbb{C}^{r^{2}}$, isomorphic to the matrix algebra $M(r)$. Let $\mathcal{A}_{r}$ be the Zariski closure of this subset. An element in $\mathcal{A}_{r}$ is called a specialization of $M(r)$. Note that there exists a locally free sheaf $W$ of $\mathcal{O}_{\mathcal{A}_{r}}$-algebras on $\mathcal{A}_{r}$ such that for each $z \in \mathcal{A}_{r},\left.W\right|_{z} \otimes \mathbb{C}$ is the specialization of $M(r)$ represented by $z$.

A family of specializations of $M(r)$ parametrized by a noetherian $\mathbb{C}$-scheme $T$ is an $\mathcal{O}_{T}$-algebra $B$ such that for all $t \in T$ there is a neighborhood $T_{1}$ of $t$ and a morphism $f: T_{1} \rightarrow \mathcal{A}_{r}$ satisfying $\left.f^{*}(W) \cong B\right|_{T_{1}}$.

Let $\mathfrak{S}_{r}$ be the functor from the category of schemes of finite type over $\mathbb{C}$ to the category of sets which to each $\mathbb{C}$-scheme $T$ associates the set of isomorphism classes of families of specializations of $M(r)$ parametrized by $T$. It is known that $\mathfrak{S}_{r}$ is represented by $\mathcal{A}_{r}$ and $\mathcal{A}_{2}$ is smooth (see [21, Chapter 5-I]).

3. Higgs bundles and parabolic Higgs bundles

In this section, we show that there exists a bijection set-theoretically between $\mathbf{M}_{r}^{s}$ and a subset of $\mathbf{M}_{r^{2},\left(a_{1}, a_{2}\right)}^{\mathrm{par}, s}$.

Proposition 3.1. There exist $a_{1}$ and $a_{2}$ such that whenever $n \leq r^{2}$,

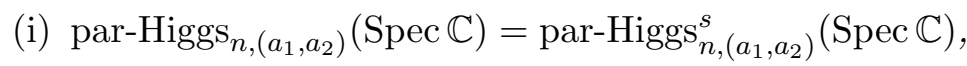


(ii) for any $(E, \phi, s) \in \operatorname{par}^{-H_{i g g s},\left(a_{1}, a_{2}\right)}(\operatorname{Spec} \mathbb{C}),(E, \phi)$ is a semistable Higgs bundle,

(iii) for any $(E, \phi, s) \in \operatorname{par}^{-H_{i g g s}}{ }_{n,\left(a_{1}, a_{2}\right)}(\operatorname{Spec} \mathbb{C})$ and any $\phi$-invariant subbundle $F$ of $E$, we have

$$
\mu(F)<0 \Longrightarrow \operatorname{par} \mu\left(\left(F, s_{F}\right)\right)<\operatorname{par} \mu((E, s))
$$

Proof. Take $a_{2}$ such that $a_{2}<\frac{1}{r^{2}}$.

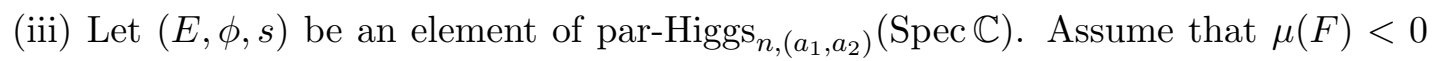
for any $\phi$-invariant subbundle $F$ of $E$.

If $\left.F\right|_{x_{0}} \not \subset F_{2}(E)$, then

$$
\operatorname{par} \mu((E, s))-\operatorname{par} \mu\left(\left(F, s_{F}\right)\right)=-\mu(F)+\frac{\left(a_{2}-a_{1}\right)(n-\operatorname{rank}(F))}{n \cdot \operatorname{rank}(F)}>0 .
$$

If $\left.F\right|_{x_{0}} \subset F_{2}(E)$, then

$$
\operatorname{par} \mu((E, s))-\operatorname{par} \mu\left(\left(F, s_{F}\right)\right)=-\mu(F)+\frac{a_{1}-a_{2}}{n} .
$$

If $-\mu(F)+\frac{a_{1}-a_{2}}{n} \leq 0$, then

$$
0<-\operatorname{deg}(F) n \leq\left(a_{2}-a_{1}\right) \operatorname{rank}(F)<\frac{\operatorname{rank}(F)}{r^{2}} \leq \frac{\operatorname{rank}(F)}{n}<1,
$$

which is a contradiction. Thus $-\mu(F)+\frac{a_{1}-a_{2}}{n}>0$.

(i) Let $(E, \phi, s)$ be an element of par-Higgs ${ }_{n,\left(a_{1}, a_{2}\right)}(\operatorname{Spec} \mathbb{C})$ and let $F$ be a $\phi$-invariant subbundle of $E$. Then we have par $\mu((E, s))-\operatorname{par} \mu\left(\left(F, s_{F}\right)\right) \geq 0$. By (iii), we have only to consider the case $\operatorname{deg}(F) \geq 0$.

If $\left.F\right|_{x_{0}} \not \subset F_{2}(E)$ and $\operatorname{par} \mu((E, s))-\operatorname{par} \mu\left(\left(F, s_{F}\right)\right)=0$ for some $\phi$-invariant proper subbundle $F$ of $E$, then $0=-\mu(F)+\frac{\left(a_{2}-a_{1}\right)(n-\operatorname{rank}(F))}{n \cdot \operatorname{rank}(F)}$. Thus since $a_{2}<\frac{1}{r^{2}} \leq \frac{1}{n}$,

$$
0=-\operatorname{deg}(F) n+\left(a_{2}-a_{1}\right)(n-\operatorname{rank}(F))<-\operatorname{deg}(F) n+\frac{n-\operatorname{rank}(F)}{n} .
$$

Since $\operatorname{deg}(F) \geq 0$, we have $0 \leq \operatorname{deg}(F) n<\frac{n-\operatorname{rank}(F)}{n}<1$, which implies $\operatorname{deg}(F)=0$. This means that $\left(a_{2}-a_{1}\right)(n-\operatorname{rank}(F))=0$, which is impossible.

If $\left.F\right|_{x_{0}} \subset F_{2}(E)$ and $\operatorname{par} \mu((E, s))-\operatorname{par} \mu\left(\left(F, s_{F}\right)\right)=0$ for some $\phi$-invariant proper subbundle $F$ of $E$, then $0=-\mu(F)+\frac{a_{1}-a_{2}}{n}$. So $-\operatorname{deg}(F) n=\left(a_{2}-a_{1}\right) \operatorname{rank}(F)>0$. But $\operatorname{deg}(F) \geq 0$, which is a contradiction.

(ii) Let $(E, \phi, s)$ be an element of par-Higgs ${ }_{n,\left(a_{1}, a_{2}\right)}(\operatorname{Spec} \mathbb{C})$ and let $F$ be a $\phi$-invariant subbundle of $E$. Then we have par $\mu((E, s))-\operatorname{par} \mu\left(\left(F, s_{F}\right)\right) \geq 0$. Assume that $\mu(F)>0$.

If $\left.F\right|_{x_{0}} \not \subset F_{2}(E)$ for some $\phi$-invariant proper subbundle $F$ of $E$, then

$$
-\mu(F)+\frac{\left(a_{2}-a_{1}\right)(n-\operatorname{rank}(F))}{n \cdot \operatorname{rank}(F)} \geq 0 .
$$


Since $a_{2}<\frac{1}{r^{2}} \leq \frac{1}{n}, 0<\operatorname{deg}(F) n \leq\left(a_{2}-a_{1}\right)(n-\operatorname{rank}(F))<\frac{n-\operatorname{rank}(F)}{n}<1$, which is a contradiction.

If $\left.F\right|_{x_{0}} \subset F_{2}(E)$ for some $\phi$-invariant proper subbundle $F$ of $E$, then $-\mu(F)+\frac{a_{1}-a_{2}}{n} \geq 0$. So $0<\mu(F) \leq \frac{a_{1}-a_{2}}{n}<0$, which is a contradiction.

Under the choice of weights of Proposition 3.1, $\mathbf{M}_{n,\left(a_{1}, a_{2}\right)}^{\mathrm{par}}=\mathbf{M}_{n,\left(a_{1}, a_{2}\right)}^{\mathrm{par},{ }^{2}}$.

Let $(E, \phi)$ be a semistable Higgs bundle of rank $n$ on $X$. It is well known that $(E, \phi)$ has a Jordan-Hölder filtration

$$
E=E_{0} \supset E_{1} \supset \cdots \supset E_{k+1}=0
$$

such that for all $i, E_{i}$ is $\phi$-invariant and $\left(E_{i} / E_{i+1}, \bar{\phi}\right)$ are stable Higgs bundles with $\mu\left(E_{i} / E_{i+1}\right)=\mu(E)$.

Lemma 3.2. Every semistable Higgs bundle $(E, \phi)$ contains a unique nontrivial maximal $\phi$-invariant subbundle $P S(E)$ of $E$ such that $\left(P S(E),\left.\phi\right|_{P S(E)}\right)$ is a polystable Higgs bundle and $\mu(P S(E))=\mu(E)$.

Proof. In a Jordan-Hölder filtration of $(E, \phi)$, there always exists a $\phi$-invariant proper subbundle $E_{1}$ such that $\left(E_{1},\left.\phi\right|_{E_{1}}\right)$ is stable and $\mu\left(E_{1}\right)=\mu(E)$. By Zorn's Lemma, there is at least one nontrivial maximal $\phi$-invariant subbundle $P S(E)$ of $E$ such that $\left(P S(E),\left.\phi\right|_{P S(E)}\right)$ is a polystable Higgs bundle and $\mu(P S(E))=\mu(E)$. Assume that there are two maximal polystable Higgs bundle $\left(P S_{1}(E),\left.\phi\right|_{P S_{1}(E)}\right)$ and $\left(P S_{2}(E),\left.\phi\right|_{P S_{2}(E)}\right)$. And assume that the uniqueness has been proved for all semistable pair $\left(E^{\prime}, \phi^{\prime}\right)$ with $\operatorname{rank}\left(E^{\prime}\right)<\operatorname{rank}(E)$. Both of $\left(P S_{1}(E),\left.\phi\right|_{P S_{1}(E)}\right)$ and $\left(P S_{2}(E),\left.\phi\right|_{P S_{2}(E)}\right)$ contain the stable Higgs bundle $\left(E_{1},\left.\phi\right|_{E_{1}}\right)$. Note that the induced Higgs bundle $\left(E / E_{1}, \phi_{E / E_{1}}\right)$ is semistable with $\mu\left(E / E_{1}\right)=\mu(E)$. Then by induction hypothesis,

$$
\begin{aligned}
\left(P S\left(E / E_{1}\right),\left.\phi_{E / E_{1}}\right|_{P S\left(E / E_{1}\right)}\right) & \cong\left(P S_{1}(E) / E_{1},\left.\phi\right|_{P S_{1}(E) / E_{1}}\right) \\
& \cong\left(P S_{2}(E) / E_{1},\left.\phi\right|_{P S_{2}(E) / E_{1}}\right)
\end{aligned}
$$

Therefore $\left(P S_{1}(E),\left.\phi\right|_{P S_{1}(E)}\right) \cong\left(P S_{2}(E),\left.\phi\right|_{P S_{2}(E)}\right)$.

Remark 3.3. [11, Lemma 1.5.5] Let $P_{\mathcal{F}}$ be the reduced Hilbert polynomial of a coherent sheaf $\mathcal{F}$ on $Z$. Let $\mathcal{E}$ be the coherent sheaf on $Z$ corresponding to $(E, \phi)$ such that $\operatorname{Supp}(\mathcal{E}) \cap D=\emptyset$ mentioned in Theorem 2.2. By the same idea of the proof of Lemma 3.2. we can show that $\mathcal{E}$ contains a unique nontrivial maximal subsheaf $P S(\mathcal{E})$ of $\mathcal{E}$ such that $P S(\mathcal{E})$ is a polystable sheaf and $P_{P S(\mathcal{E})}=P_{\mathcal{E}}$.

Then we have the following series of consequences. 
Proposition 3.4. Let $(E, \phi)$ be a semistable Higgs bundle of rank $n$ on $X$. Then there exists a section s such that $(E, \phi, s) \in \operatorname{par}^{-H_{i g g s}^{s}}{ }_{n,\left(a_{1}, a_{2}\right)}(\operatorname{Spec} \mathbb{C})$ if and only if for all stable Higgs bundles $(F, \psi)$ such that $\mu(F)=\mu(E)$ and all integers $t>\operatorname{rank}(F)$ there is no injective morphism of Higgs bundles $i:\left(F^{\oplus t}, \psi^{\oplus t}\right) \hookrightarrow(E, \phi)$.

Proof. We follow the idea of the proof of [21, Proposition 7, Chapter 5]. The proof of the if part is nontrivial. Suppose that for all stable Higgs bundles $(F, \psi)$ such that $\mu(F)=\mu(E)$, all integers $t>0$ and all injective morphism of Higgs bundles $i:\left(F^{\oplus t}, \psi^{\oplus t}\right) \hookrightarrow(E, \phi)$, we have $t \leq \operatorname{rank}(F)$.

It suffices to show that for all $\phi$-invariant subbundle $F$ of $E$ such that $\left(F,\left.\phi\right|_{F}\right)$ is a stable Higgs bundle and $\mu(F)=\mu(E)$, there exists a hyperplane $F_{2}(E)$ of $\left.E\right|_{x_{0}}$ satisfying that $\left.F\right|_{x_{0}} \not \subset F_{2}(E)$ and $\phi$ is parabolic.

Assume that the previous statement is true. If $F$ is a $\phi$-invariant proper subbundle of $E$ such that $\mu(F)<\mu(E)$, then

$$
\operatorname{par} \mu((E, s))-\operatorname{par} \mu\left(\left(F, s_{F}\right)\right)=-\mu(F)+\frac{\left(a_{2}-a_{1}\right)(n-\operatorname{rank}(F))}{n \cdot \operatorname{rank}(F)}>0 .
$$

If $F$ is a $\phi$-invariant proper subbundle of $E$ such that $\mu(F)=\mu(E)$ and $\left(F,\left.\phi\right|_{F}\right)$ is a semistable Higgs bundle, then there exists a hyperplane $F_{2}(E)$ of $\left.E\right|_{x_{0}}$ such that $\left.F\right|_{x_{0}} \not \subset$ $F_{2}(E)$ and $\phi$ is parabolic, because $\left(F,\left.\phi\right|_{F}\right)$ has a stable Higgs subbundle $\left(F^{\prime},\left.\phi\right|_{F^{\prime}}\right)$ such that $\mu\left(F^{\prime}\right)=\mu(E)$. In this case, we have also

$$
\operatorname{par} \mu((E, s))-\operatorname{par} \mu\left(\left(F, s_{F}\right)\right)=\frac{\left(a_{2}-a_{1}\right)(n-\operatorname{rank}(F))}{n \cdot \operatorname{rank}(F)}>0 .
$$

It remains to find the hyperplane $F_{2}(E)$ of $\left.E\right|_{x_{0}}$ satisfying that $\left.F\right|_{x_{0}} \nsubseteq F_{2}(E)$ and $\phi$ is parabolic, which is equivalent to find the $\phi$-invariant subsheaf $\iota_{x_{0}, *}\left(F_{2}(E)\right)$ of the skyscraper sheaf $\iota_{x_{0}, *}\left(\left.E\right|_{x_{0}}\right)$ such that $\iota_{x_{0}, *}\left(\left.F\right|_{x_{0}}\right) \nsubseteq \iota_{x_{0}, *}\left(F_{2}(E)\right)$ where $\iota_{x_{0}, *}:\left\{x_{0}\right\} \hookrightarrow X$ is the inclusion and $F_{2}(E)$ is a hyperplane of $\left.E\right|_{x_{0}}$. Note that $\iota_{x_{0}, *}\left(\left.E\right|_{x_{0}}\right)$ is of pure dimension 0 .

Let $\mathcal{E}$ be the coherent sheaf of pure dimension 1 on $Z$ corresponding to $(E, \phi)$ such that $\operatorname{Supp}(\mathcal{E}) \cap D=\emptyset$ mentioned in Theorem 2.2. Write $\left(P S(E),\left.\phi\right|_{P S(E)}\right)=\bigoplus_{i=1}^{m}\left(F_{i}^{\oplus s_{i}}, \psi_{i}^{\oplus s_{i}}\right)$ where $\left(F_{i}, \psi_{i}\right)$ is a stable pair satisfying $\mu\left(F_{i}\right)=\mu(E)$ and $\left(F_{i}, \psi_{i}\right) \neq\left(F_{j}, \psi_{j}\right)$ for $i \neq j$. Then, by Lemma 3.2 and Remark 3.3 , every stable Higgs subbundle $\left(F,\left.\phi\right|_{F}\right)$ of $(E, \phi)$ such that $\mu(F)=\mu(E)$ is isomorphic to $\left(F_{k}, \psi_{k}\right)$ for some $k$ and that the corresponding stable subsheaf $\mathcal{F}$ of $\mathcal{E}$ on $Z$ such that $P_{\mathcal{F}}=P_{\mathcal{E}}$ is isomorphic to $\mathcal{F}_{k}$. We may assume that $\left(F,\left.\phi\right|_{F}\right)=\left(F_{k}, \psi_{k}\right)$ and $\mathcal{F}=\mathcal{F}_{k}$. Let $f=\pi^{-1}\left(x_{0}\right) \backslash D$. By Lemma 2.3 . $\pi_{*}\left(\iota_{f, *}\left(\left.\mathcal{E}\right|_{f}\right)\right)=\iota_{x_{0}, *}\left(\left.E\right|_{x_{0}}\right)$ where $\iota_{f}: f \hookrightarrow\left|K_{X}\right|$ is the inclusion of $f$ and $\iota_{x_{0}}:\left\{x_{0}\right\} \hookrightarrow X$ is the inclusion of $\left\{x_{0}\right\}$. This means that $\iota_{f, *}\left(\left.\mathcal{E}\right|_{f}\right)$ is the coherent sheaf of pure dimension 0 on $Z$ corresponding to $\left(\iota_{x_{0}, *}\left(\left.E\right|_{x_{0}}\right), \iota_{x_{0}, *}\left(\left.\phi\right|_{x_{0}}\right)\right)$. Let

$$
S_{k}:=\left\{(H, \mathcal{F}) \in \mathbb{P}\left(\left.\mathcal{E}\right|_{f} ^{\vee}\right) \times \mathbb{P}\left(\mathbb{C}^{s_{k}}\right)|\mathcal{F}|_{\operatorname{Supp}\left(\iota_{f, *}\left(\left.\mathcal{E}\right|_{f}\right)\right)} \subset H\right\}
$$


Let $Z_{k}:=p r_{1}\left(S_{k}\right)$. Then $\operatorname{codim}_{\mathbb{P}\left(\left.\mathcal{E}\right|_{f} ^{\vee}\right)} Z_{k}=\operatorname{codim}_{\mathbb{P}\left(\left.\mathcal{E}\right|_{f} ^{\vee}\right)} \operatorname{pr}_{1}\left(p r_{2}^{-1}(\mathcal{F}) \cap S_{k}\right)=\operatorname{rank}\left(F_{k}\right) \geq s_{k}$. So we can take a hyperplane $H$ in $\bigcap_{k=1}^{m}\left(\mathbb{P}\left(\left.\mathcal{E}\right|_{f} ^{\vee}\right) \backslash Z_{k}\right)$. Let $\widetilde{F_{2}(E)}=\pi_{*} \iota_{f, *}(H)$. Let $\lambda: \mathcal{E} \rightarrow \mathcal{E} \otimes \pi^{*} K_{X}$ be the morphism given by the tautological section $\lambda$ of $\pi^{*} K_{X}$. By 2 , Proposition 3.6], $\phi=\pi_{*}(\lambda): E \rightarrow E \otimes K_{X}$. Since $\iota_{f, *}(H)$ is a coherent sheaf of $\mathcal{O}_{Z^{-}}$ module such that $\operatorname{Supp}\left(\iota_{f, *}(H)\right) \cap D=\emptyset, \pi_{*} \iota_{f, *}(H)$ is $S^{\bullet}\left(K_{X}^{\vee}\right)$-module and then it induces $\pi_{*}(\lambda): \pi_{*} \iota_{f, *}(H) \rightarrow \pi_{*} \iota_{f, *}(H) \otimes K_{X}$ (see [22, Lemma 2.13]). So

$$
\phi\left(\widetilde{F_{2}(E)}\right)=\pi_{*}(\lambda)\left(\pi_{*} \iota_{f, *}(H)\right) \subset \pi_{*} \iota_{f, *}(H) \otimes K_{X}=\widetilde{F_{2}(E)} \otimes K_{X} .
$$

Hence $\widetilde{F_{2}(E)}$ is a $\phi$-invariant subsheaf of $\iota_{x_{0}, *}\left(\left.E\right|_{x_{0}}\right)$ such that $\iota_{x_{0}, *}\left(\left.F\right|_{x_{0}}\right) \nsubseteq \widetilde{F_{2}(E)}$, which completes the proof.

Proposition 3.5. Let $(E, \phi, s)$ be an element of par-Higgs $n_{n,\left(a_{1}, a_{2}\right)}^{s}(\operatorname{Spec} \mathbb{C})$ and let $M(r)$ be the $\mathbb{C}$-algebra of $r \times r$ complex matrices. Then $\operatorname{dim}_{\mathbb{C}} \operatorname{End}((E, \phi)) \leq n$. Moreover when $n=r^{2}, \operatorname{End}((E, \phi)) \cong M(r)$ of $\mathbb{C}$-algebras if and only if there exists a stable Higgs bundle $(F, \psi)$ such that

$$
(E, \phi) \cong\left(F^{\oplus \operatorname{rank}(F)}, \psi^{\oplus \operatorname{rank}(F)}\right) .
$$

Proof. We follow the idea of the proof of [21, Proposition 8, Chapter 5].

Proposition 3.6. Let $\left(E_{1}, \phi_{1}, s_{1}\right),\left(E_{2}, \phi_{2}, s_{2}\right)$ be elements of

$$
\text { par-Higgs }{ }_{n,\left(a_{1}, a_{2}\right)}^{s}(\operatorname{Spec} \mathbb{C})
$$

such that

$$
\operatorname{dim} \operatorname{End}\left(\left(E_{1}, \phi_{1}\right)\right)=\operatorname{dim} \operatorname{End}\left(\left(E_{2}, \phi_{2}\right)\right)=n .
$$

Then $\left(E_{1}, \phi_{1}, s_{1}\right) \cong\left(E_{2}, \phi_{2}, s_{2}\right)$ if and only if $\left(E_{1}, \phi_{1}\right) \cong\left(E_{2}, \phi_{2}\right)$.

Proof. We follow the idea of the proof of [21, Proposition 9, Chapter 5]. The only if part is obvious. For the proof of the if part, suppose that $(E, \phi)$ is a semistable Higgs bundle of rank $n$ on $X$ which corresponds to a semistable coherent sheaf $\mathcal{E}$ such that $\operatorname{Supp}(\mathcal{E}) \cap D=\emptyset$. Choose two $\phi$-invariant hyperplanes $H_{1}$ and $H_{2}$ of $\left.\mathcal{E}\right|_{f}$ that make $(E, \phi)$ stable parabolic Higgs bundles $\left(E_{1}, \phi_{1}, s_{1}\right)$ and $\left(E_{2}, \phi_{2}, s_{2}\right)$ respectively. The group $\operatorname{Aut}_{\mathcal{O}_{Z}}(\mathcal{E})=\operatorname{Aut}_{\mathcal{O}_{X}}((E, \phi))$ acts on

$$
\bigcap_{i=1}^{m}\left(\mathbb{P}\left(\left.\mathcal{E}\right|_{f} ^{\vee}\right) \backslash Z_{i}\right) .
$$

Since every stable parabolic Higgs bundle is simple, the stabilizer of a point in $\bigcap_{i=1}^{m}\left(\mathbb{P}\left(\left.\mathcal{E}\right|_{f} ^{\vee}\right)\right.$

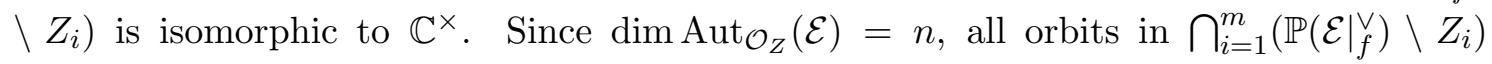
have dimension $n-1$. Thus $\operatorname{Aut}_{\mathcal{O}_{Z}}(\mathcal{E})$ acts on $\bigcap_{i=1}^{m}\left(\mathbb{P}\left(\left.\mathcal{E}\right|_{f} ^{\vee}\right) \backslash Z_{i}\right)$ transitively. Therefore $H_{1}=g^{*} H_{2}$ for some $g \in \operatorname{Aut}_{\mathcal{O}_{Z}}(\mathcal{E})$, that is, $H_{1}$ and $H_{2}$ give an isomorphism of parabolic Higgs bundles $\left(E_{1}, \phi_{1}, s_{1}\right)$ and $\left(E_{2}, \phi_{2}, s_{2}\right)$. 
As a result, we have the following result.

Theorem 3.7. Assume that $a_{2}<\frac{1}{r^{2}}$ as Proposition 3.1. Let

$$
\begin{array}{c|c}
\mathbf{S}^{\prime}:=\left\{[(E, \phi, s)] \in \mathbf{M}_{r^{2},\left(a_{1}, a_{2}\right)}^{\mathrm{par}}=\mathbf{M}_{r^{2},\left(a_{1}, a_{2}\right)}^{\mathrm{par},} \mid\right. & \operatorname{End}((E, \phi)) \cong M(r), \operatorname{det} E \cong \mathcal{O}_{X} \\
\text { and } \phi \text { is traceless }\} .
\end{array}
$$

Then there exists a bijection

$$
\mathbf{M}_{r}^{s} \rightarrow \mathbf{S}^{\prime}, \quad(F, \psi) \mapsto\left(F^{\oplus r}, \psi^{\oplus r}, s_{\text {can }}\right)
$$

set-theoretically where $s_{\mathrm{can}}$ is a canonical section so that $\left(F^{\oplus r}, \psi^{\oplus r}, s_{\mathrm{can}}\right) \in \mathbf{M}_{r^{2},\left(a_{1}, a_{2}\right)}^{\mathrm{par}, \mathrm{s}}$ from Proposition 3.4 .

Proof. The definition and well-definedness is obtained from Propositions 3.4, 3.5 and 3.6. The surjectivity follows from Proposition 3.5. The injectivity follows from Proposition 3.6.

\section{Kirwan's desingularization of $\mathbf{M}_{2}$}

In this section, we briefly show that $\mathbf{M}_{2}$ is desingularized by three blow-ups by Kirwan's algorithm in the sense of [14]. For more details, see [13, Section 4].

In [22, Theorems 3.8 and 4.10], C. T. Simpson showed that a good quotient R// SL(2) is $\mathbf{M}_{2}$ where $\mathbf{R}$ is an irreducible normal quasi-projective variety which represents the moduli functor which parameterizes triples $(V, \phi, \beta)$ where $(V, \phi)$ is a semistable Higgs bundle with $\operatorname{det} V \cong \mathcal{O}_{X}, \operatorname{tr} \phi=0$ and $\beta$ is an isomorphism

$$
\beta:\left.V\right|_{x} \rightarrow \mathbb{C}^{2}
$$

and he also showed that every point in $\mathbf{R}$ is semistable with respect to the action of $\mathrm{SL}(2)$, that the closed orbits in $\mathbf{R}$ correspond to polystable Higgs bundles $(V, \phi)$, that is, stable or $(V, \phi)=(L, \psi) \oplus\left(L^{-1},-\psi\right)$ for $L \in \operatorname{Pic}^{0}(X)$ and $\psi \in H^{0}\left(K_{X}\right)$ and that the set $\mathbf{R}^{s}$ of stable points with respect to the action of $\mathrm{SL}(2)$ is exactly the locus of stable Higgs bundles.

We need to review a deformation theory for Higgs bundles discussed in [22, Section 10] and [13, Section 4]. $A^{i}$ denotes the sheaf of smooth $i$-forms on $X$. For a polystable Higgs bundle $(V, \phi)$, we have the following complex

$$
0 \longrightarrow \mathcal{E} n d_{0} V \otimes A^{0} \stackrel{\bar{\partial}+\phi}{\longrightarrow} \mathcal{E} n d_{0} V \otimes A^{1} \stackrel{\bar{\partial}+\phi}{\longrightarrow} \mathcal{E} n d_{0} V \otimes A^{2} \longrightarrow 0 .
$$


This complex induces a long exact sequence

$$
0 \longrightarrow T^{0} \longrightarrow H^{0}\left(\mathcal{E} n d_{0} V\right) \stackrel{[\phi,-]}{\longrightarrow} H^{0}\left(\mathcal{E} n d_{0} V \otimes K_{X}\right)
$$

$$
\longrightarrow T^{1} \longrightarrow H^{1}\left(\mathcal{E} n d_{0} V\right) \stackrel{[\phi,-]}{\longrightarrow} H^{1}\left(\mathcal{E} n d_{0} V \otimes K_{X}\right) \longrightarrow T^{2} \longrightarrow 0
$$

where $T^{i}$ is the $i$-th cohomology of (4.1). Here the Zariski tangent space of $\mathbf{M}_{2}$ at a polystable Higgs bundle $(V, \phi)$ is isomorphic to $T^{1}$. To investigate the singularities of $\mathbf{M}_{2}$, we must recall the following theorem proved by C. T. Simpson.

Theorem 4.1. 22, Theorems 10.4 and 10.5] Let $C$ be the quadratic cone in $T^{1}$ defined by the map $T^{1} \rightarrow T^{2}$ which sends an $\mathcal{E} n d_{0} V$-valued 1 -form $\eta$ to $[\eta, \eta]$. Let $y=(V, \phi, \beta) \in \mathbf{R}$ be a point with closed orbit and $\bar{y} \in \mathbf{M}_{2}$ the point coming from $y$. Then the formal completion $(\mathbf{R}, y)^{\wedge}$ is isomorphic to the formal completion $\left(C \times \mathfrak{h}^{\perp}, 0\right)^{\wedge}$ where $\mathfrak{h}^{\perp}$ is the perpendicular space to the image of the evaluation at $x$ composed with $\beta, T^{0} \rightarrow H^{0}\left(\mathcal{E} n d_{0} V\right) \rightarrow \operatorname{sl}(2)$. Furthermore, if $Y$ is the étale slice at $y$ of the $\operatorname{SL}(2)$-orbit in $\mathbf{R}$, then $(Y, y)^{\wedge} \cong(C, 0)^{\wedge}$ and $\left(\mathbf{M}_{2}, \bar{y}\right)^{\wedge}=(Y / / \operatorname{Stab}(y), \bar{y})^{\wedge} \cong(C / / \operatorname{Stab}(y), v)^{\wedge}$ where $\operatorname{Stab}(y)$ is the stabilizer of $y$ and $v$ is the cone point of $C$.

Let $\Omega_{\mathbf{R}}$ (resp. $\Omega_{\mathbf{M}_{2}}$ ) be the locus of $(L, 0) \oplus(L, 0)$ for $L \cong L^{-1}$ in $\mathbf{R} \backslash \mathbf{R}^{s}$ (resp. in $\left.\mathbf{M}_{2} \backslash \mathbf{M}_{2}^{s}\right)$. Both $\Omega_{\mathbf{R}}$ and $\Omega_{\mathbf{M}_{2}}$ are isomorphic to the $\mathbb{Z}_{2}$-fixed point set $\mathbb{Z}_{2}^{2 g}$ in $J:=\operatorname{Pic}^{0}(X)$ by the involution $L \mapsto L^{-1}$. By 4.2 , we have isomorphisms

$$
\begin{gathered}
T^{0} \cong H^{0}\left(\mathcal{E} n d_{0} V\right) \cong \operatorname{sl}(2), \\
T^{1} \cong H^{0}\left(\mathcal{E} n d_{0} V \otimes K_{X}\right) \oplus H^{1}\left(\mathcal{E} n d_{0} V\right) \cong H^{0}\left(K_{X}\right) \otimes \operatorname{sl}(2) \oplus H^{1}\left(\mathcal{O}_{X}\right) \otimes \operatorname{sl}(2)
\end{gathered}
$$

and

$$
T^{2} \cong H^{1}\left(\mathcal{E} n d_{0} V \otimes K_{X}\right) \cong H^{1}\left(K_{X}\right) \otimes \operatorname{sl}(2) \cong \operatorname{sl}(2) .
$$

Then by Theorem 4.1, the normal cone of $\Omega_{\mathbf{M}_{2}}$ in $\mathbf{M}_{2}$ is a locally trivial fibration over $\Omega_{\mathbf{M}_{2}}$ with fiber

$$
\bar{\Upsilon}^{-1}(0) / / \operatorname{SL}(2)
$$

where $\bar{\Upsilon}: H^{0}\left(K_{X}\right) \otimes \operatorname{sl}(2) \oplus H^{1}\left(\mathcal{O}_{X}\right) \otimes \operatorname{sl}(2) \rightarrow H^{1}\left(K_{X}\right) \otimes \operatorname{sl}(2) \cong \operatorname{sl}(2)$ is the quadratic map given by $(\alpha \otimes a, \beta \otimes b) \mapsto(\alpha \cup \beta) \otimes[a, b]$, the Lie bracket of $\operatorname{sl}(2)$ coupled with the perfect pairing $H^{0}\left(K_{X}\right) \oplus H^{1}\left(\mathcal{O}_{X}\right) \rightarrow H^{1}\left(K_{X}\right)$.

Let $\Sigma_{\mathbf{R}}$ (resp. $\Sigma_{\mathbf{M}_{2}}$ ) be the locus of $(L, \psi) \oplus\left(L^{-1},-\psi\right)$ for $(L, \psi) \varsubsetneqq\left(L^{-1},-\psi\right)$ in $\mathbf{R} \backslash \mathbf{R}^{s}$ (resp. in $\mathbf{M}_{2} \backslash \mathbf{M}_{2}^{s}$ ). $\Sigma_{\mathbf{M}_{2}}$ is isomorphic to

$$
J \times_{\mathbb{Z}_{2}} H^{0}\left(K_{X}\right)-\mathbb{Z}_{2}^{2 g} \cong T^{*} J / \mathbb{Z}_{2}-\mathbb{Z}_{2}^{2 g}
$$


where $\mathbb{Z}_{2}$ acts on $T^{*} J$ by $(L, \psi) \mapsto\left(L^{-1},-\psi\right)$. Since $\Sigma_{\mathbf{R}}$ is a $\mathbb{P} \operatorname{SL}(2) / \mathbb{C}^{\times}$-bundle over $T^{*} J / \mathbb{Z}_{2}-\mathbb{Z}_{2}^{2 g}$, it is smooth. Since $L ¥ L^{-1}, H^{0}\left(\mathcal{E} n d_{0} V\right) \cong H^{0}\left(\mathcal{O}_{X}\right)$ and then $[\phi,-]$ : $H^{0}\left(\mathcal{E} n d_{0} V\right) \rightarrow H^{0}\left(\mathcal{E} n d_{0} V \otimes K_{X}\right)$ is zero. Then by $(4.2)$, we have isomorphisms

$$
\begin{aligned}
& T^{0} \cong \operatorname{ker}\left([\phi,-]: H^{0}\left(\mathcal{E} n d_{0} V\right) \rightarrow H^{0}\left(\mathcal{E} n d_{0} V \otimes K_{X}\right)\right) \cong H^{0}\left(\mathcal{O}_{X}\right) \cong \mathbb{C}, \\
T^{1} \cong & \operatorname{coker}\left([\phi,-]: H^{0}\left(\mathcal{E} n d_{0} V\right) \rightarrow H^{0}\left(\mathcal{E} n d_{0} V \otimes K_{X}\right)\right) \\
& \oplus \operatorname{ker}\left([\phi,-]: H^{1}\left(\mathcal{E} n d_{0} V\right) \rightarrow H^{1}\left(\mathcal{E} n d_{0} V \otimes K_{X}\right)\right) \\
\cong & H^{0}\left(\mathcal{E} n d_{0} V \otimes K_{X}\right) \oplus H^{1}\left(\mathcal{E} n d_{0} V\right) \\
\cong & {\left[H^{1}\left(\mathcal{O}_{X}\right) \oplus H^{0}\left(K_{X}\right)\right] \oplus\left[H^{0}\left(L^{-2} K_{X}\right) \oplus H^{0}\left(L^{2} K_{X}\right) \oplus H^{1}\left(L^{2}\right) \oplus H^{1}\left(L^{-2}\right)\right] }
\end{aligned}
$$

and

$$
T^{2} \cong \operatorname{coker}\left([\phi,-]: H^{1}\left(\mathcal{E} n d_{0} V\right) \rightarrow H^{1}\left(\mathcal{E} n d_{0} V \otimes K_{X}\right)\right) \cong H^{1}\left(\mathcal{E} n d_{0} V \otimes K_{X}\right) \cong H^{1}\left(K_{X}\right) .
$$

Note that $H^{1}\left(\mathcal{O}_{X}\right) \oplus H^{0}\left(K_{X}\right)$ is the Zariski tangent space of $T^{*} J / \mathbb{Z}_{2}-\mathbb{Z}_{2}^{2 g}$ at any point. Then by Theorem 4.1, the normal cone of $\Sigma_{\mathbf{M}_{2}}$ in $\mathbf{M}_{2}$ is a locally trivial fibration over $\Sigma_{\mathbf{M}_{2}}$ with fiber

$$
\bar{\Psi}^{-1}(0) / / \mathbb{C}^{\times}
$$

where $\bar{\Psi}: H^{0}\left(L^{-2} K_{X}\right) \oplus H^{0}\left(L^{2} K_{X}\right) \oplus H^{1}\left(L^{2}\right) \oplus H^{1}\left(L^{-2}\right) \rightarrow H^{1}\left(K_{X}\right)$ is the quadratic map given by $(a, b, c, d) \mapsto a \cup c+b \cup d$ for $(L, \psi) \oplus\left(L^{-1},-\psi\right) \in T^{*} J / \mathbb{Z}_{2}-\mathbb{Z}_{2}^{2 g}$ and for perfect pairings $\cup: H^{0}\left(L^{-2} K_{X}\right) \oplus H^{1}\left(L^{2}\right) \rightarrow H^{1}\left(K_{X}\right)$ and $\cup: H^{0}\left(L^{2} K_{X}\right) \oplus H^{1}\left(L^{-2}\right) \rightarrow H^{1}\left(K_{X}\right)$. Here the definition of $\bar{\Psi}$ comes from the diagonal entry in $\left[\left(\begin{array}{cc}* & b \\ a & -*\end{array}\right),\left(\begin{array}{cc}*^{\prime} & c \\ d & -*^{\prime}\end{array}\right)\right]$.

On the other hand, we have a stratification of $\mathbf{M}_{2}$ :

$$
\mathbf{M}_{2}=\mathbf{M}_{2}^{s} \sqcup\left(T^{*} J / \mathbb{Z}_{2}-\mathbb{Z}_{2}^{2 g}\right) \sqcup \mathbb{Z}_{2}^{2 g} .
$$

Since we have identical singularities and stratification as in O'Grady's case in [19], we can adapt his arguments to construct the Kirwan's desingularization $\mathbf{K}$ of $\mathbf{M}_{2}$ and its blow-downs.

Let $\widetilde{\mathbf{R}}$ be the variety obtained by blowing up $\mathbf{R}$ first along $\Omega_{\mathbf{R}}$ and then along the strict transform of $\Sigma_{\mathbf{R}}$. Let $\widetilde{\mathbf{R}}^{s s}$ (resp. $\widetilde{\mathbf{R}}^{s}$ ) be the locus of semistable (resp. stable) points of $\widetilde{\mathbf{R}}$. Then we have (a) $\widetilde{\mathbf{R}}^{s s}=\widetilde{\mathbf{R}}^{s}$, (b) $\widetilde{\mathbf{R}}^{s}$ is smooth. By blowing up $\widetilde{\mathbf{R}}^{s}$ one more time along the locus $\widetilde{\Delta}$ of points with stabilizers larger than the center $\mathbb{Z}_{2}$ of SL(2), we obtain a variety $\widehat{\mathbf{R}}$ with a smooth orbit space

$$
\mathbf{K}:=\widehat{\mathbf{R}} / \mathrm{SL}(2)
$$

obtained by blowing up $\mathbf{M}_{2}$ first along $\mathbb{Z}_{2}^{2 g}$, second along the strict transform of $T^{*} J / \mathbb{Z}_{2}$ and third along the nonsingular subvariety $\widetilde{\Delta} / / \mathrm{SL}(2)$ contained in the strict transform of the exceptional divisor of the first blow-up. Let

$$
\pi_{\mathbf{K}}: \mathbf{K} \rightarrow \mathbf{M}_{2}
$$


be the composition of these three blow-ups. $\mathbf{K}$ is called the Kirwan's desingularization of $\mathbf{M}_{2}$.

5. The construction of a desingularization of $\mathbf{M}_{2}$

In this section, we construct a desingularization of $\mathbf{M}_{2}$. Assume that $r=2$ and fix $a_{2}<\frac{1}{4}$ as Proposition 3.1. Then $\mathbf{M}_{4,\left(a_{1}, a_{2}\right)}^{\mathrm{par}}=\mathbf{M}_{4,\left(a_{1}, a_{2}\right)}^{\mathrm{par}, \mathrm{s}}$. Let par-Higgs $\mathrm{H}_{4,\left(a_{1}, a_{2}\right)}^{s p}$ be the subfunctor of par-Higgs ${ }_{4,\left(a_{1}, a_{2}\right)}$ consisting of all families of stable parabolic Higgs bundles such that for each $\mathbb{C}$-scheme $T$ and each $(\mathcal{E}, \varphi, \sigma) \in$ par-Higgs $_{4,\left(a_{1}, a_{2}\right)}(T), p_{T *} \mathcal{E} n d((\mathcal{E}, \varphi)) \in \mathfrak{S}_{2}(T)$, where $p_{T}: X \times T \rightarrow T$ is the projection.

\subsection{Formally smoothness of par-Higgs $s_{4,\left(a_{1}, a_{2}\right)}^{s p}$}

In this subsection, we prove that par-Higgs ${ }_{4,\left(a_{1}, a_{2}\right)}^{s p}$ is formally smooth. Let $\mathcal{A}$ be the category of local artinian commutative $\mathbb{C}$-algebras with residue field $\mathbb{C}$. Let $\mathcal{F}$ and $\mathcal{G}$ be covariant functors from $\mathcal{A}$ to the category of sets.

Definition 5.1. (1) A morphism of functors $f: \mathcal{F} \rightarrow \mathcal{G}$ is called formally smooth if given any surjective homomorphism $p: A^{\prime} \rightarrow A$ in $\mathcal{A}$ with the kernel $I$ such that $\mathfrak{m}_{A^{\prime}} I=0$ and any elements $\alpha \in \mathcal{F}(A)$ and $\beta \in \mathcal{G}\left(A^{\prime}\right)$ such that

$$
f_{A}(\alpha)=\mathcal{G}(p)(\beta) \in \mathcal{G}(A),
$$

there exists an element $\gamma \in \mathcal{F}\left(A^{\prime}\right)$ such that

$$
f_{A^{\prime}}(\gamma)=\beta \in \mathcal{G}\left(A^{\prime}\right) \quad \text { and } \quad \mathcal{F}(p)(\gamma)=\alpha \in \mathcal{F}(A) .
$$

(2) A functor $\mathcal{F}$ is called formally smooth if $\mathcal{F}(p): \mathcal{F}\left(A^{\prime}\right) \rightarrow \mathcal{F}(A)$ is surjective for any small surjection $p: A^{\prime} \rightarrow A$ in $\mathcal{A}$.

From the definition of par-Higgs $s_{4,\left(a_{1}, a_{2}\right)}^{s p}$, we have the morphism of functors

$$
\Phi: \operatorname{par}^{-H_{i g g s}}{ }_{4,\left(a_{1}, a_{2}\right)}^{s p} \rightarrow \mathfrak{S}_{2}
$$

given by $\Phi_{T}$ : $\operatorname{par}^{-H_{i g g s}}{ }_{4,\left(a_{1}, a_{2}\right)}^{s p}(T) \rightarrow \mathfrak{S}_{2}(T),(\mathcal{E}, \varphi, \sigma) \mapsto p_{T *} \mathcal{E} n d((\mathcal{E}, \varphi))$ for each $\mathbb{C}$ scheme $T$.

We claim that $\Phi$ is formally smooth, which implies that par-Higgs $s_{4,\left(a_{1}, a_{2}\right)}^{s p}$ is formally smooth as a corollary.

Lemma 5.2. Let par-Higgs end lf $_{4,\left(a_{1}, a_{2}\right)}$ be the subfunctor of par-Higgs $4_{4,\left(a_{1}, a_{2}\right)}$ consisting of all families of stable parabolic Higgs bundles such that for each $\mathbb{C}$-scheme $T$ and each $(\mathcal{E}, \varphi, \sigma) \in$ par-Higgs $_{4,\left(a_{1}, a_{2}\right)}(T), p_{T *} \mathcal{E} n d((\mathcal{E}, \varphi))$ is locally free, where $p_{T}: X \times T \rightarrow T$ is the projection. Then par-Higgs $\mathrm{H}_{4,\left(a_{1}, a_{2}\right)}^{\mathrm{end} \text { lf }}$ is an open subfunctor of par-Higgs ${ }_{4,\left(a_{1}, a_{2}\right)}$. 
Proof. For any $(\mathcal{E}, \varphi, \sigma) \in \operatorname{par}^{-H_{i g g s},\left(a_{1}, a_{2}\right)}(T)$, let $U=\left\{t \in T \mid\left(p_{T *} \mathcal{E} n d((\mathcal{E}, \varphi))\right)_{t}\right.$ is locally free $\}$ which is an open subscheme of $T$. Then we can see that for any $f: T^{\prime} \rightarrow T$, we have

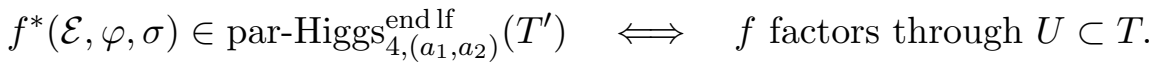

Lemma 5.3. The open subfunctor par-Higgs $\mathrm{end}_{4,\left(a_{1}, a_{2}\right)}$ of par-Higgs $_{4,\left(a_{1}, a_{2}\right)}$ defined as in Lemma 5.2 is represented by an open subscheme of $\mathbf{M}_{4,\left(a_{1}, a_{2}\right)}^{\mathrm{par}}$.

Proof. By Proposition 5.7, par-Higgs ${ }_{4,\left(a_{1}, a_{2}\right)}$ is represented by $\mathbf{M}_{4,\left(a_{1}, a_{2}\right)}^{\text {par }}$. Let $\left(\mathcal{E}^{\text {univ }}, \varphi^{\text {univ }}\right.$, $\left.\sigma^{\text {univ }}\right)$ be the universal family. Consider $U^{\text {univ }}=\left\{t \in \mathbf{M}_{4,\left(a_{1}, a_{2}\right)}^{\text {par }} \mid\left(p_{\mathbf{M}_{4,\left(a_{1}, a_{2}\right)}{ }^{\text {par }}} \mathcal{E} n d\left(\left(\mathcal{E}^{\text {univ }}\right.\right.\right.\right.$, $\left.\left.\left.\varphi^{\text {univ }}\right)\right)\right)_{t}$ is locally free $\}$ which is an open subscheme of $\mathbf{M}_{4,\left(a_{1}, a_{2}\right)}^{\mathrm{par}}$. We can see that par-Higgs ${ }_{4,\left(a_{1}, a_{2}\right)}^{\text {end lf }}$ is represented by $U^{\text {univ }}$.

Lemma 5.4. The open subfunctor par-Higgs $\mathrm{end}_{4,\left(a_{1}, a_{2}\right)}^{\text {end }}$ par-Higgs pa, $_{\left.4, a_{1}, a_{2}\right)}$ defined as in Lemma 5.2 is formally smooth.

Proof. Since the open immersion $i: U^{\text {univ }} \hookrightarrow \mathbf{M}_{4,\left(a_{1}, a_{2}\right)}^{\text {par }}$ is smooth, the morphism of functors par-Higgs $\mathrm{e}_{4,\left(a_{1}, a_{2}\right)} \rightarrow$ par-Higgs $_{4,\left(a_{1}, a_{2}\right)}$ induced from $i$ is formally smooth by Lemma 5.3 and the relative version of the infinitesimal lifting property (see [6, Exercise 4.7]).

Since we have chosen $a_{1}$ and $a_{2}$ such that $\mathbf{M}_{4,\left(a_{1}, a_{2}\right)}^{\mathrm{par}}=\mathbf{M}_{4,\left(a_{1}, a_{2}\right)}^{\mathrm{par}, s}$ in Proposition 3.1 and $\mathbf{M}_{4,\left(a_{1}, a_{2}\right)}^{\mathrm{par}, s}$ is smooth,

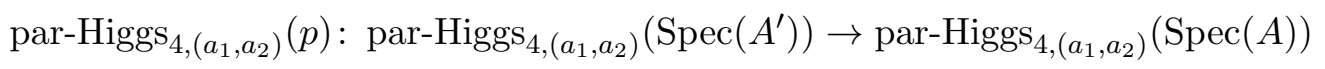

is surjective for any surjective homomorphism $p: A^{\prime} \rightarrow A$ in $\mathcal{A}$ with the kernel $I$ such that $\mathfrak{m}_{A^{\prime}} I=0$ by [24, Proposition 2.2, Theorems 2.4 and 5.2]. Hence we get the conclusion.

Proposition 5.5. The morphism of functors

$$
\Phi: \operatorname{par}^{-H_{i g g s}}{ }_{4,\left(a_{1}, a_{2}\right)} \rightarrow \mathfrak{S}_{2}
$$

is formally smooth.

Proof. Let $(E, \phi, s) \in \operatorname{par}^{-H_{i g g s}}{ }_{4,\left(a_{1}, a_{2}\right)}^{s p}(\operatorname{Spec}(A))$ and $B^{\prime} \in \mathfrak{S}_{2}\left(\operatorname{Spec}\left(A^{\prime}\right)\right)$ such that $p_{A *} \mathcal{E} n d((E, \phi)) \cong \iota^{*} B^{\prime}$ where $p: A^{\prime} \rightarrow A$ is a surjective homomorphism in $\mathcal{A}$ with the kernel $I$ such that $\mathfrak{m}_{A^{\prime}} I=0, \iota: \operatorname{Spec}(A) \hookrightarrow \operatorname{Spec}\left(A^{\prime}\right)$ is the inclusion and $p_{A}: X \times$ $\operatorname{Spec}(A) \rightarrow \operatorname{Spec}(A)$ is the projection. Note that $(E, \phi, s) \in \operatorname{par}^{-H_{i g g s}}$ end lf $_{\left.4, a_{1}, a_{2}\right)}(\operatorname{Spec}(A))$. It suffices to show that there exists a family of parabolic Higgs bundles $\left(E^{\prime}, \phi^{\prime}, s^{\prime}\right) \in$ par-Higgs $\operatorname{Hat}_{4,\left(a_{1}, a_{2}\right)}^{s p}\left(\operatorname{Spec}\left(A^{\prime}\right)\right)$ such that $p_{A^{\prime} *} \mathcal{E} n d\left(\left(E^{\prime}, \phi^{\prime}\right)\right) \cong B^{\prime}$ and $\iota^{*}\left(E^{\prime}, \phi^{\prime}, s^{\prime}\right) \cong(E, \phi, s)$, where $p_{A^{\prime}}: X \times \operatorname{Spec}\left(A^{\prime}\right) \rightarrow \operatorname{Spec}\left(A^{\prime}\right)$ is the projection.

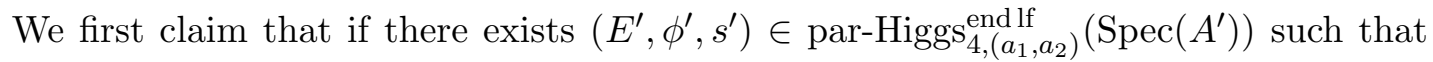
$\iota^{*}\left(E^{\prime}, \phi^{\prime}, s^{\prime}\right) \cong(E, \phi, s)$, then $p_{A^{\prime} *} \mathcal{E} n d\left(\left(E^{\prime}, \phi^{\prime}\right)\right) \cong B^{\prime}$. Since $B^{\prime}=f^{*} W$ for some morphism 
$f: \operatorname{Spec}\left(A^{\prime}\right) \rightarrow \mathcal{A}_{2}, B^{\prime}$ is locally free, that is, projective. Since we have the following commutative diagram

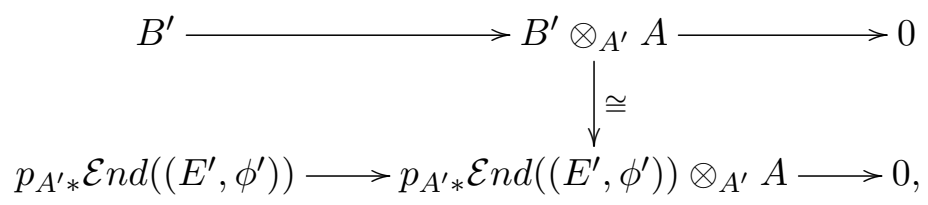

we get a surjective morphism $s: B^{\prime} \rightarrow p_{A^{\prime} *} \mathcal{E} n d\left(\left(E^{\prime}, \phi^{\prime}\right)\right)$. Since $p_{A^{\prime} *} \mathcal{E} n d\left(\left(E^{\prime}, \phi^{\prime}\right)\right)$ is locally free by the hypothesis and the rank of $B^{\prime}$ is equal to the rank of $p_{A^{\prime} *} \mathcal{E} n d\left(\left(E^{\prime}, \phi^{\prime}\right)\right), s: B^{\prime} \rightarrow$ $p_{A^{\prime} *} \mathcal{E} n d\left(\left(E^{\prime}, \phi^{\prime}\right)\right)$ must be an isomorphism.

Next we show that there exists $\left(E^{\prime}, \phi^{\prime}, s^{\prime}\right) \in \operatorname{par}^{-H_{i g g s}} \sin _{4,\left(a_{1}, a_{2}\right)}\left(\operatorname{Spec}\left(A^{\prime}\right)\right)$ such that $\iota^{*}\left(E^{\prime}, \phi^{\prime}, s^{\prime}\right) \cong(E, \phi, s)$. This is an immediate consequence of Lemma 5.4 .

Corollary 5.6. par-Higgs ${ }_{4,\left(a_{1}, a_{2}\right)}^{s p}$ is formally smooth.

Proof. For any surjective homomorphism $p: A^{\prime} \rightarrow A$ in $\mathcal{A}$ with the kernel $I$ such that $\mathfrak{m}_{A} I=0$, we have the following commutative diagram

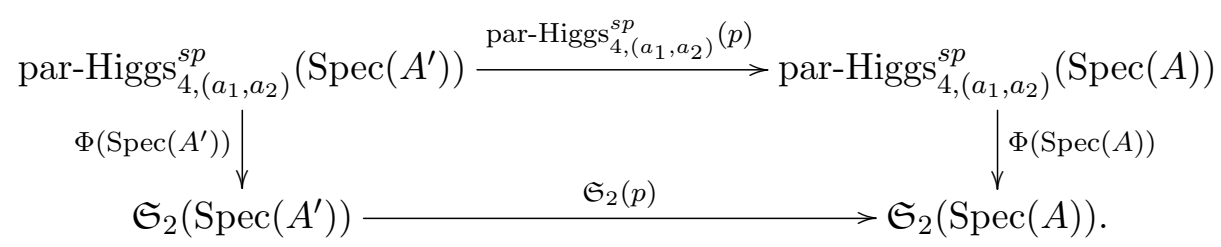

Since $\mathcal{A}_{2}$ is smooth, $\mathfrak{S}_{2}(p)$ is surjective. Combining this with Proposition 5.5 . $\operatorname{par-Higgs}_{4,\left(a_{1}, a_{2}\right)}^{s p}(p)$ is surjective.

\subsection{Fine moduli scheme of par-Higgs $s_{4,\left(a_{1}, a_{2}\right)}^{s p}$}

In this subsection, we construct a fine moduli scheme $\mathbf{S}$ of par-Higgs $\mathrm{s}_{4,\left(a_{1}, a_{2}\right)}^{s p}$ as a closed subvariety of $\mathbf{M}_{4,\left(a_{1}, a_{2}\right)}^{\mathrm{par}}$. Then we conclude that $\mathbf{S}$ is indeed a desingularization of $\mathbf{M}_{2}$.

Proposition 5.7. $\mathbf{M}_{4,\left(a_{1}, a_{2}\right)}^{\mathrm{par}}$ is a fine moduli scheme of par-Higgs ${ }_{4,\left(a_{1}, a_{2}\right)}$.

Proof. Step 1. We first claim that $\mathbf{M}_{4,\left(a_{1}, a_{2}\right)}^{\mathrm{par}}=\mathbf{M}_{4,\left(a_{1}, a_{2}\right)}^{\mathrm{par}, s}=\mathcal{R}^{s} / \mathrm{SL}_{\nu(m)}=\mathcal{R}^{s} / \mathrm{PGL}_{\nu(m)}$ for some quasi-projective variety $\mathcal{R}$ with $\mathrm{SL}_{\nu(m)}$-action and $m \gg 0$, where $\nu(m)=n m+$ $n(1-g)$ and $\mathcal{R}^{s} / \mathrm{SL}_{\nu(m)}$ is the moduli space of 1-stable parabolic Higgs bundles of degree 0 and rank 4 on $X$ with multiplicities $(1,3)$ and weights $\left(a_{1}, a_{2}\right)$ constructed in [23, $\left.\S 2\right]$. It suffices to show that every stable parabolic Higgs bundle $(E, \phi, s)$ is 1-stable (see 23 , Definition 1.7]), that is, every nonzero subbundle $F$ of $E$ has $\operatorname{deg}(F) \leq \operatorname{rank}(F)$.

Let $F_{2}(E)$ be the hyperplane given by $s$. Let $F$ be a subbundle of $E$. If $\left.F\right|_{x_{0}} \not \subset F_{2}(E)$, then

$$
\operatorname{par} \mu((E, s))-\operatorname{par} \mu\left(\left(F, s_{F}\right)\right)=-\mu(F)+\frac{\left(a_{2}-a_{1}\right)(4-\operatorname{rank}(F))}{4 \cdot \operatorname{rank}(F)}>0,
$$


which implies that

$$
\operatorname{deg}(F)<\operatorname{rank}(F) \frac{\left(a_{2}-a_{1}\right)(4-\operatorname{rank}(F))}{4 \cdot \operatorname{rank}(F)}<\operatorname{rank}(F) \frac{4-\operatorname{rank}(F)}{4}<\operatorname{rank}(F) .
$$

If $\left.F\right|_{x_{0}} \subset F_{2}(E)$, then

$$
\operatorname{par} \mu((E, s))-\operatorname{par} \mu\left(\left(F, s_{F}\right)\right)=-\mu(F)+\frac{a_{1}-a_{2}}{4}>0,
$$

which implies that $\operatorname{deg}(F)<\operatorname{rank}(F) \frac{a_{1}-a_{2}}{4}<\operatorname{rank}(F)$.

Step 2. Let $\mathcal{U}$ be the universal family on $X \times \mathcal{R}, \mathcal{U}^{s}=\left.\mathcal{U}\right|_{X \times \mathcal{R}^{s}}$ and let $\pi_{X}: X \times \mathcal{R}^{s} \rightarrow X$ and $\pi_{\mathcal{R}^{s}}: X \times \mathcal{R}^{s} \rightarrow \mathcal{R}^{s}$ be the projections onto the first and the second factor respectively. Note that $\mathrm{GL}_{\nu(m)}$-action on $\mathcal{R}^{s}$ lifts to an action on $\mathcal{U}^{s}$ and $\lambda(\mathrm{id})$ acts on $\mathcal{U}$ by scalar multiplication by $\lambda$ in fibers. If we are given a line bundle $L$ on $\mathcal{R}^{s}$ such that $\mathrm{GL}_{\nu(m)^{-}}$ action on $\mathcal{R}^{s}$ lifts to an action on $L$ and $\lambda(\mathrm{id})$ acts on $L$ by scalar multiplication by $\lambda$, then $\mathcal{U}^{s} \otimes \pi_{\mathcal{R}^{s}}^{*} L^{-1}$ descends to the desired universal family on $X \times \mathbf{M}_{4,\left(a_{1}, a_{2}\right)}^{\mathrm{par}, s}$ by the descent lemma due to Kempf.

We have only to construct $L$ mentioned above. Define $\chi(k)=-4(2 g-2)+m_{k}$. Let $\left(E, \phi, s_{E}\right)$ be a parabolic Higgs bundle in par-Higgs $s_{4,\left(a_{1}, a_{2}\right)}$ and let $\left(H_{k}, \psi_{k}, s_{H_{k}}\right)$ be a parabolic line Higgs bundle with weights $a_{k}$ for $k=1,2$. Then

$$
\begin{gathered}
\operatorname{dim} \mathbf{H}^{0}\left(\mathbf{H o m}\left(\left(H_{k}, \psi_{k}, s_{H_{k}}\right),\left(E, \phi, s_{E}\right)\right)\right)-\operatorname{dim} \mathbf{H}^{1}\left(\mathbf{H o m}\left(\left(H_{k}, \psi_{k}, s_{H_{k}}\right),\left(E, \phi, s_{E}\right)\right)\right) \\
+\operatorname{dim} \mathbf{H}^{2}\left(\mathbf{H o m}\left(\left(H_{k}, \psi_{k}, s_{H_{k}}\right),\left(E, \phi, s_{E}\right)\right)\right)=\chi(k)
\end{gathered}
$$

by Theorem 2.9, Let

$$
\begin{aligned}
L(k)= & \operatorname{det} \mathbf{R}^{0} \pi_{R^{s}, *} \operatorname{Hom}\left(\pi_{X}^{*}\left(H_{k}, \psi_{k}, s_{H_{k}}\right), \mathcal{U}^{s}\right) \\
& \otimes \operatorname{det} \mathbf{R}^{1} \pi_{R^{s}, *} \operatorname{Hom}\left(\pi_{X}^{*}\left(H_{k}, \psi_{k}, s_{H_{k}}\right), \mathcal{U}^{s}\right)^{-1} \\
& \otimes \operatorname{det} \mathbf{R}^{2} \pi_{R^{s}, *} \operatorname{Hom}\left(\pi_{X}^{*}\left(H_{k}, \psi_{k}, s_{H_{k}}\right), \mathcal{U}^{s}\right) .
\end{aligned}
$$

$\mathrm{GL}_{\nu(m)}$-action on $\mathcal{U}$ induces a $\mathrm{GL}_{\nu(m)}$-action on $L(k)$ and $\lambda(\mathrm{id})$ acts on $L(k)$ by scalar multiplication by $\lambda^{\chi(k)}$. Since $\chi(1)$ and $\chi(2)$ are consecutive odd numbers, they are relatively prime. Thus there exist $c_{1}, c_{2} \in \mathbb{Z}$ such that $c_{1} \chi(1)+c_{2} \chi(2)=1$. Hence $L:=L(1)^{c_{1}} \otimes L(2)^{c_{2}}$ is the desired line bundle on $\mathcal{R}^{s}$.

The following is the construction of $\mathbf{S}$.

Proposition 5.8. Let $\mathbf{S}$ be the subset of $\mathbf{M}_{4,\left(a_{1}, a_{2}\right)}^{\mathrm{par}}$ consisting of stable parabolic Higgs bundles $(E, \phi, s)$ such that $\operatorname{End}((E, \phi))$ is a specialization of $M(2)$, $\operatorname{det} E \cong \mathcal{O}_{X}$ and $\phi$ is traceless. Then

(1) $\mathbf{S}$ is a closed subvariety of $\mathbf{M}_{4,\left(a_{1}, a_{2}\right)}^{\mathrm{par}}$. 
(2) $\mathbf{S}$ is a fine moduli scheme of par-Higgs ${ }_{4,\left(a_{1}, a_{2}\right)}^{s p}$.

Proof. (1) The proof is identical as that of [21, Theorem 15-(i), Chapter 5]. Let $\mathbf{S}^{\prime}$ be the subset of $\mathbf{S}$ consisting of stable parabolic Higgs bundles $(E, \phi, s)$ such that $\operatorname{End}((E, \phi)) \cong$ $M(2)$ and $\operatorname{det} E \cong \mathcal{O}_{X}$. It suffices to show that $\mathbf{S}=\overline{\mathbf{S}^{\prime}}$.

Let $(E, \phi, s)$ be an element of $\mathbf{S}$. Let $\mathcal{E}$ be the coherent sheaf of pure dimension 1 on $Z$ corresponding to $(E, \phi)$ such that $\operatorname{Supp}(\mathcal{E}) \cap D=\emptyset$ mentioned in Theorem 2.2 .

Since $\mathcal{A}_{2}=\overline{\mathcal{A}_{2}^{\prime}}$, there exists a $\mathcal{O}_{\operatorname{Spec}(\mathbb{C}[[T]])}$-algebra $B$ as an element of $\mathfrak{S}_{2}(\operatorname{Spec}(\mathbb{C}[[T]]))$ such that

$$
B \otimes_{\mathbb{C}[[T]]} \mathbb{C} \cong \operatorname{End}((E, \phi)) \quad \text { and } \quad B \otimes_{\mathbb{C}[[T]]} \overline{\mathbb{C}((T))} \cong M(2, \overline{\mathbb{C}((T))})
$$

where $\mathbb{C}((T))$ is the field of fractions of $\mathbb{C}[[T]]$ and $\overline{\mathbb{C}((T))}$ is the algebraic closure of $\mathbb{C}((T))$.

If we are given a flat family $(\mathcal{F}, \psi, \sigma)$ as an element of

$$
\operatorname{par}^{-H_{i g g s}}{ }_{4,\left(a_{1}, a_{2}\right)}^{s p}(\operatorname{Spec}(\mathbb{C}[[T]]))
$$

such that $B \cong p_{0 *} \mathcal{E} n d((\mathcal{F}, \psi))$ and $i^{*}(\mathcal{F}, \psi, \sigma) \cong(E, \phi, s)$, where $p_{0}: X \times \operatorname{Spec}(\mathbb{C}[[T]]) \rightarrow$ $\operatorname{Spec}(\mathbb{C}[[T]])$ is the projection, $i: \operatorname{Spec}(\mathbb{C}) \hookrightarrow \operatorname{Spec}(\mathbb{C}[[T]])$ is the inclusion, it gives us $\mathbf{S} \subset \overline{\mathbf{S}^{\prime}}$ by $\left[5\right.$, Theorem of 3.1]. It is obvious that $\overline{\mathbf{S}^{\prime}} \subset \mathbf{S}$.

It remains to prove the existence of $(\mathcal{F}, \psi, \sigma)$. Let $D_{n}:=\mathbb{C}[[T]] /\left(T^{n}\right)$ for all integer $n \geq 1$. This is an element of $\mathcal{A}$. By Proposition 5.5, there exists a sequence $\left(\mathcal{F}_{n}, \psi_{n}, \sigma_{n}\right)_{n \geq 1}$ such that for each integer $n \geq 1$,

(a) $\left(\mathcal{F}_{n}, \psi_{n}, \sigma_{n}\right)$ is an element of par-Higgs ${ }_{4,\left(a_{1}, a_{2}\right)}^{s p}\left(\operatorname{Spec}\left(D_{n}\right)\right)$,

(b) there exists an isomorphism $g_{n}: i_{n}^{*}\left(\mathcal{F}_{n+1}, \psi_{n+1}, \sigma_{n+1}\right) \rightarrow\left(\mathcal{F}_{n}, \psi_{n}, \sigma_{n}\right)$ where $i_{n}: \operatorname{Spec}\left(D_{n}\right) \hookrightarrow \operatorname{Spec}\left(D_{n+1}\right)$ is the inclusion,

(c) there exists an isomorphism $f_{n}: p_{n *} \mathcal{E} n d\left(\left(\mathcal{F}_{n}, \psi_{n}\right)\right) \rightarrow j_{n}^{*} B$ such that $f_{n+1}$ induces $f_{n}$ via $g_{n}$ where $p_{n}: X \times \operatorname{Spec}\left(D_{n}\right) \rightarrow \operatorname{Spec}\left(D_{n}\right)$ is the projection and $j_{n}: \operatorname{Spec}\left(D_{n}\right) \hookrightarrow$ $\operatorname{Spec}(\mathbb{C}[[T]])$ is the inclusion.

Then there exists a family of Higgs bundles $(\mathcal{F}, \psi)$ uniquely on $X \times \operatorname{Spec}(\mathbb{C}[[T]])$ such that, for each integer $n \geq 1$, we get an isomorphism $h_{n}: j_{n}^{*}(\mathcal{F}, \psi) \rightarrow\left(\mathcal{F}_{n}, \psi_{n}\right)$ such that $h_{n+1}$ induces $h_{n}$ via $g_{n}$. Precisely, let $U=\operatorname{Spec}(A)$ be an affine open subset of $X$. $\left(\left.\mathcal{F}_{n}\right|_{U \times \operatorname{Spec}\left(D_{n}\right)}, \psi_{n}\right)_{n \geq 1}$ on $X$ corresponds to $\left(\left.\widetilde{\mathcal{F}}_{n}\right|_{\pi^{-1}(U) \times \operatorname{Spec}\left(D_{n}\right)}\right)_{n \geq 1}$ on $Z$ in the sense of Theorem 2.2. This sequence is equivalent to the datum of a sequence $\left(M_{n}\right)_{n \geq 1}$ such that for each integer $n \geq 1$,

(a) $M_{n}$ is a finitely generated $A \otimes_{\mathbb{C}} D_{n}$-module,

(b) there exists an isomorphism $M_{n+1} \otimes_{A \otimes_{\mathbb{C}} D_{n+1}}\left(A \otimes_{\mathbb{C}} D_{n}\right) \rightarrow M_{n}$. 
Put $M=\lim _{n} M_{n}$ which is a finitely generated $A \otimes_{\mathbb{C}} \mathbb{C}[[T]]$-module. Then we get a coherent sheaf $\widetilde{\mathcal{F}}$ on $Z \times \operatorname{Spec}(\mathbb{C}[[T]])$ which corresponds to a pair $(\mathcal{F}, \psi)$ as desired.

Sections of $\left.\widetilde{\mathcal{F}}_{n}^{\vee}\right|_{f \times \operatorname{Spec}\left(D_{n}\right)}$ induced from $\sigma_{n}$ can be extended to a section $\sigma$ of $\left.\widetilde{\mathcal{F}}^{\vee}\right|_{\operatorname{Spec}(f \times \mathbb{C}[[T]])}$ such that $(\mathcal{F}, \psi, \sigma) \in \operatorname{par} \operatorname{Higgs}_{4,\left(a_{1}, a_{2}\right)}(\operatorname{Spec}(\mathbb{C}[[T]]))$, and isomorphisms $f_{n}: p_{n *} \mathcal{E} n d\left(\left(\mathcal{F}_{n}, \psi_{n}\right)\right) \rightarrow j_{n}^{*} B$ gives an isomorphism $f: p_{0 *} \mathcal{E} n d((\mathcal{F}, \psi)) \rightarrow B$ by taking inverse limits.

(2) By Proposition 5.7, $\mathbf{M}_{4,\left(a_{1}, a_{2}\right)}^{\text {par }}$ is fine. Then we can adapt the proof of 21, Theorem 15-(ii), Chapter 5].

Then we get the first main result.

Theorem 5.9. (Theorem 1.1) $\mathbf{S}$ is nonsingular. Furthermore, there is a canonical morphism $\pi_{\mathbf{S}}: \mathbf{S} \rightarrow \mathbf{M}_{2}$ which extends the isomorphism $\mathbf{S}^{\prime} \stackrel{\cong}{\rightarrow} \mathbf{M}_{2}^{s}$ given by $(E, \phi, s) \mapsto(F, \psi)$ where $(F, \psi)$ is the uniquely determined stable Higgs bundle such that $(E, \phi) \cong(F, \psi) \oplus$ $(F, \psi)$.

Proof. The first statement is an immediate consequence from Corollary 5.6 and Proposition 5.8 .

Let's prove the second statement. Let $g: \mathbf{M}_{2} \rightarrow \mathbf{M}_{4}$ be a morphism given by $\operatorname{gr}((F, \psi))$ $\mapsto \operatorname{gr}((F, \psi) \oplus(F, \psi))$, where $\operatorname{gr}((F, \psi))$ and $\operatorname{gr}((F, \psi) \oplus(F, \psi))$ are graded objects associated to Jordan-Hölder filtrations of $(F, \psi)$ and $(F, \psi) \oplus(F, \psi)$ respectively. It is obvious that $g$ is injective. Since $g$ induces an isomorphism between $\mathbf{M}_{2}^{s}$ and $g\left(\mathbf{M}_{2}^{s}\right), g: \mathbf{M}_{2} \rightarrow g\left(\mathbf{M}_{2}\right)$ is birational. Indeed, $g$ is a homeomorphism onto $g\left(\mathbf{M}_{2}\right)$ because $\mathbf{M}_{2} \backslash \mathbf{M}_{2}^{s}=T^{*} J / \mathbb{Z}_{2}$ (see $\$ 4$ is separated (i.e., the diagonal morphism $\Delta: T^{*} J / \mathbb{Z}_{2} \rightarrow T^{*} J / \mathbb{Z}_{2} \times T^{*} J / \mathbb{Z}_{2}$ is a closed immersion) and $g\left(\mathbf{M}_{2}\right) \backslash g\left(\mathbf{M}_{2}^{s}\right)$ is homeomorphic to $\Delta\left(T^{*} J / \mathbb{Z}_{2}\right)$.

Since $g\left(\mathbf{M}_{2}\right)$ and $p(\mathbf{S})$ contain the common dense subset $g\left(\mathbf{M}_{2}^{s}\right)$ from Theorem 3.7, we have $g\left(\mathbf{M}_{2}\right)=p(\mathbf{S})$, where $p$ denotes the canonical forgetful morphism $\mathbf{M}_{4,\left(a_{1}, a_{2}\right)}^{\text {par }} \rightarrow \mathbf{M}_{4}$. Let $p^{\prime}=\left.p\right|_{\mathbf{S}}$.

Since $\mathbf{S}$ is nonsingular, the continuous function $g^{-1} \circ p^{\prime}: \mathbf{S} \stackrel{p^{\prime}}{\rightarrow} g\left(\mathbf{M}_{2}\right) \stackrel{g^{-1}}{\longrightarrow} \mathbf{M}_{2}$ is a morphism $\pi_{\mathbf{S}}: \mathbf{S} \rightarrow \mathbf{M}_{2}$ by GAGA theorem and Riemann's extension theorem (see $[16,4.10])$. It is an immediate consequence from the construction that $\pi_{\mathbf{S}}$ induces an isomorphism between $\pi_{\mathbf{S}}^{-1}\left(\mathbf{M}_{2}^{s}\right)$ and $\mathbf{M}_{2}^{s}$.

6. A comparison between $\mathbf{S}$ and the Kirwan's desingularization of $\mathbf{M}_{2}$

In this section we solve a comparison problem, naturally raised from Theorem 5.9 , between $\mathbf{S}$ and the Kirwan's desingularization of $\mathbf{M}_{2}$. 


\subsection{A comparison between $\mathbf{S}$ and $\mathbf{K}$}

By Proposition 7.5, K have consecutive contractions

$$
f: \mathbf{K} \stackrel{f_{\sigma}}{\longrightarrow} \mathbf{K}_{\sigma} \stackrel{f_{\epsilon}}{\longrightarrow} \mathbf{K}_{\epsilon}
$$

which are blow-downs. The following is the second main result which is a solution of this comparison problem.

Theorem 6.1. (Theorem 1.2$) \mathbf{K}_{\epsilon} \cong \mathbf{S}$.

\section{A proof of Theorem 6.1}

In this section we first construct the morphism $\rho: \mathbf{K} \rightarrow \mathbf{S}$, show that $\rho$ factors through $\mathbf{K}_{\epsilon}$, that is,

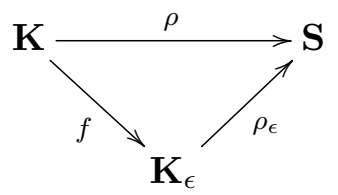

and then we finally conclude that $\mathbf{K}_{\epsilon} \cong \mathbf{S}$.

\subsection{A morphism from $\mathbf{K}$ to $\mathbf{S}$}

We begin with the following proposition, which will be useful later.

Proposition 7.1. Let $x_{0} \in X$.

(1) Let $(E, \phi) \rightarrow T \times X$ be a family of semistable Higgs bundles of rank 4 and degree 0 on $X$ parametrized by a complex manifold $T$. Assume the following:

(a) for any $t \in T$, any line bundle $L$ of degree 0 on $X$ and any $\psi \in H^{0}\left(K_{X}\right)$, $(L, \psi) \oplus(L, \psi)$ is not isomorphic to a Higgs subbundle of $\left(\left.E\right|_{t \times X},\left.\phi\right|_{t \times X}\right)$.

(b) there is an open dense subset $T^{\prime}$ of $T$ such that

$$
\operatorname{End}\left(\left(\left.E\right|_{t \times X},\left.\phi\right|_{t \times X}\right)\right) \cong M(2)
$$

for any $t \in T^{\prime}$.

Then we have a holomorphic map $\tau: T \rightarrow \mathbf{S}$.

(2) Suppose a holomorphic map $\tau: T \rightarrow \mathbf{S}$ is given. Suppose that $T$ is an open subset of a nonsingular quasi-projective variety $W$ on which a reductive group $G$ acts such that every point in $W$ is stable and the (smooth) geometric quotient $q: W \rightarrow W / G$ exists. Furthermore, assume that there is an open dense subset $W^{\prime}$ of $W$ such that 
whenever $t_{1}, t_{2} \in T \cap W^{\prime}$ are in the same orbit, we have $\tau\left(t_{1}\right)=\tau\left(t_{2}\right)$. Then $\tau$ factors through the image $\bar{T}$ of $T$ in $W / G$, that is, there is a continuous map $\bar{T} \rightarrow \mathbf{S}$ such that the following diagram

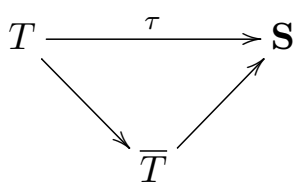

commutes.

Proof. (1) Let $\left(E_{t}, \phi_{t}\right)=\left(\left.E\right|_{t \times X},\left.\phi\right|_{t \times X}\right)$. By (a) and Proposition 3.4, there exists a section $0 \neq s_{t}$ such that $\left(E_{t}, \phi_{t}, s_{t}\right)$ is a stable parabolic Higgs bundle for each $t \in T$. Since $\mathbf{M}_{4,\left(a_{1}, a_{2}\right)}^{\text {par }}$ is a coarse moduli scheme of par-Higgs ${\text { s, }\left(a_{1}, a_{2}\right)}_{\text {by }}$ Theorem 2.8 , we obtain a holomorphic map $\tau: T \rightarrow \mathbf{M}_{4,\left(a_{1}, a_{2}\right)}^{\text {par }}$. By (b), $\tau\left(T^{\prime}\right) \subset \mathbf{S}^{\prime}$ and then $\tau$ is a map into $\mathbf{S}$.

(2) This is an immediate consequence of the étale slice theorem. In particular, $\bar{T}$ is an open subset of $W / G$ in the usual complex topology.

Since both $\mathbf{K}$ and $\mathbf{S}$ contain Zariski open dense subsets which are isomorphic to $\mathbf{M}_{2}^{s}$, there is a rational map

$$
\rho^{\prime}: \mathbf{K} \rightarrow \mathbf{S}
$$

Proposition 7.2. There is a birational morphism $\rho: \mathbf{K} \rightarrow \mathbf{S}$ that extends $\rho^{\prime}$.

Proof. The strategy of the proof is similar to that of 12. By GAGA theorem and Riemann's extension theorem (see $[16,4.10]$ ), it is enough to prove that $\rho^{\prime}$ is extended to a continuous map with respect to the usual complex topology. Note that $\mathbf{M}_{2}=\mathbf{R} / / \mathrm{SL}(2)$ for some irreducible normal quasi-projective variety $\mathbf{R}$ mentioned in $\$ 4$. Here $\mathbf{R}$ is a fine moduli scheme of a moduli functor (see $\$ 4$ or $[22$ for more details). Luna's slice theorem tells us that for each point $x \in \mathbf{M}_{2} \backslash \mathbf{M}_{2}^{s}$, there is an analytic submanifold $W$ of $\mathbf{R}$ such that $W / H$ is analytically equivalent to a neighborhood of $x$ in $\mathbf{M}_{2}$, where $H$ is the stabilizer of a point in the intersection of $W$ and the closed orbit represented by $x$. Further, we can see that Kirwan's desingularization $\widetilde{W} / / H$ of $W / / H$ is a neighborhood of the preimage of $x$ in K. By Proposition 7.1, it is sufficient to construct a nice family of semistable Higgs bundles of rank 4 parametrized by $\widetilde{W}$ by successive applications of elementary modifications of Higgs bundles, beginning with a family of rank 2 Higgs bundles parametrized by $W$, which comes from the universal family over $X \times \mathbf{R}$. Then we get a classifying morphism $\widetilde{W} \rightarrow \mathbf{S}$ because $\mathbf{S}$ is the fine moduli scheme. Since this is invariant under the $H$-action, we have an induced morphism $\widetilde{W} / / H \rightarrow \mathbf{S}$. Therefore, $\rho^{\prime}$ is extended to a neighborhood of the preimage of $x$ in $\mathbf{K}$. The detail of the construction of the morphism $\widetilde{W} \rightarrow \mathbf{S}$ can be found in $\$ 7.1 .1-7.1 .5$. 


\subsubsection{Points over the middle stratum}

We first extend $\rho^{\prime}$ to points over the middle stratum of $\mathbf{M}_{2}$. Let $l=\left[(L, \psi) \oplus\left(L^{-1},-\psi\right)\right] \in$ $T^{*} J / \mathbb{Z}_{2}-\mathbb{Z}_{2}^{2 g} \subset \mathbf{M}_{2}$ and let $\mathcal{N}_{l}=H^{0}\left(K_{X}\right) \oplus H^{1}\left(\mathcal{O}_{X}\right) \oplus \bar{\Psi}^{-1}(0)$. By Theorem 4.1, the universal object over $X \times \mathbf{R}$ induces a holomorphic family $\left(\mathcal{F}, \varphi_{\mathcal{F}}\right)$ of semistable Higgs bundles over $X$ parametrized by a neighborhood $U_{l}$ of 0 in $\mathcal{N}_{l}$. The restriction of $\left(\mathcal{F}, \varphi_{\mathcal{F}}\right)$ to $X \times\left(U_{l} \cap\left(H^{0}\left(K_{X}\right) \oplus H^{1}\left(\mathcal{O}_{X}\right)\right)\right)$ is of the form $\left(\mathcal{L}, \psi_{\mathcal{L}}\right) \oplus\left(\mathcal{L}^{-1},-\psi_{\mathcal{L}}\right)$ where $\mathbf{R}\left(\mathbb{C}^{\times}\right)$ is a quasi-projective variety parametrizing degree 0 Higgs line bundles $(L, \psi)$ with an isomorphism $\beta:\left.L\right|_{x} \rightarrow \mathbb{C}($ see $[22])$ and $\left(\mathcal{L}, \psi_{\mathcal{L}}\right)$ is a family of Higgs bundles of rank 1 and degree 0 that comes from an étale map between $H^{0}\left(K_{X}\right) \oplus H^{1}\left(\mathcal{O}_{X}\right)$ and the slice in $\mathbf{R}\left(\mathbb{C}^{\times}\right)$.

Let $\pi_{l}: \widetilde{\mathcal{N}}_{l} \rightarrow \mathcal{N}_{l}$ be the blow-up of $\mathcal{N}_{l}$ along $H^{0}\left(K_{X}\right) \oplus H^{1}\left(\mathcal{O}_{X}\right)$. Let $\widetilde{U}_{l}=\pi_{l}^{-1}\left(U_{l}\right) \cap \widetilde{\mathcal{N}}_{l}^{s s}$ and $D_{l}$ be the exceptional locus in $\widetilde{U}_{l}$. Let $\left(\widetilde{\mathcal{F}}, \varphi_{\widetilde{\mathcal{F}}}\right)$ and $\left(\widetilde{\mathcal{L}}, \psi_{\widetilde{\mathcal{L}}}\right)$ be the pull-backs of $\left(\mathcal{F}, \varphi_{\mathcal{F}}\right)$ and $\left(\mathcal{L}, \psi_{\mathcal{L}}\right)$ to $\widetilde{U}_{l}$ and $D_{l}$ respectively. Then there are surjective morphisms

$$
\left(\left.\widetilde{\mathcal{F}}\right|_{D_{l}}, \varphi_{\left.\widetilde{\mathcal{F}}\right|_{D_{l}}}\right) \rightarrow\left(\widetilde{\mathcal{L}}, \psi_{\mathcal{L}}\right) \quad \text { and } \quad\left(\left.\widetilde{\mathcal{F}}\right|_{D_{l}}, \varphi_{\left.\widetilde{\mathcal{F}}\right|_{D_{l}}}\right) \rightarrow\left(\widetilde{\mathcal{L}}^{-1},-\psi_{\mathcal{L}}\right)
$$

Let $\widetilde{\mathcal{F}}^{\prime}$ and $\widetilde{\mathcal{F}}^{\prime \prime}$ be the kernels of $\left.\widetilde{\mathcal{F}} \rightarrow \widetilde{\mathcal{F}}\right|_{D_{l}} \rightarrow \widetilde{\mathcal{L}},\left.\widetilde{\mathcal{F}} \rightarrow \widetilde{\mathcal{F}}\right|_{D_{l}} \rightarrow \widetilde{\mathcal{L}}^{-1}$ respectively. Then $\varphi_{\widetilde{\mathcal{F}}^{\prime}}: \widetilde{\mathcal{F}}^{\prime} \rightarrow \widetilde{\mathcal{F}}^{\prime} \otimes p_{1}^{*} K_{X}$ and $\varphi_{\widetilde{\mathcal{F}}^{\prime \prime}}: \widetilde{\mathcal{F}}^{\prime \prime} \rightarrow \widetilde{\mathcal{F}}^{\prime \prime} \otimes p_{1}^{*} K_{X}$ can be determined uniquely such that

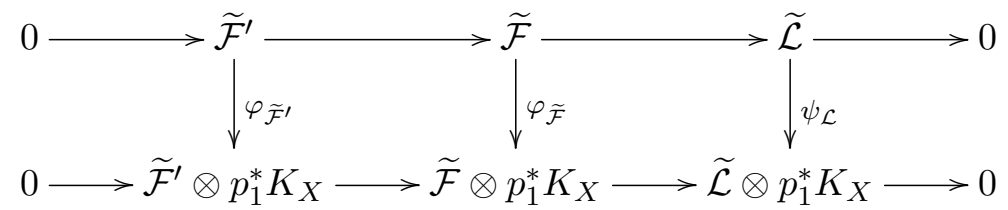

and

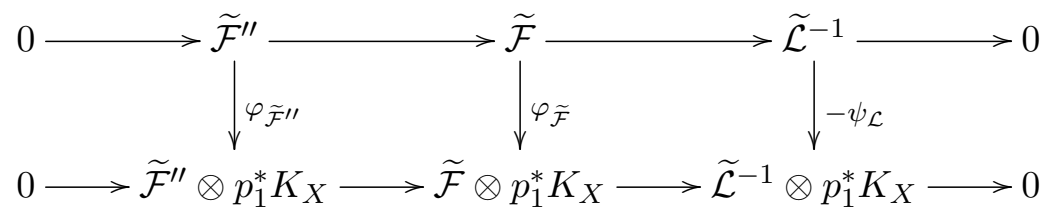

commute. Consider $\left(\mathcal{E}, \varphi_{\mathcal{E}}\right)=\left(\widetilde{\mathcal{F}}^{\prime}, \varphi_{\widetilde{\mathcal{F}}^{\prime}}\right) \oplus\left(\widetilde{\mathcal{F}}^{\prime \prime}, \varphi_{\widetilde{\mathcal{F}}^{\prime \prime}}\right)$ over $X \times \widetilde{U}_{l}$. The following lemma tells us that $\left(\mathcal{E}, \varphi_{\mathcal{E}}\right)$ satisfies the assumptions of Proposition 7.1 .

Lemma 7.3. $\left(\mathcal{E}, \varphi_{\mathcal{E}}\right)$ is a family of semistable Higgs bundles of rank 4 , degree 0 over $X$ parametrized by $\widetilde{U}_{l}$ such that

(1) for each $t \in \widetilde{U}_{l}, L^{\prime} \in \operatorname{Pic}^{0}(X)$ and $\psi^{\prime} \in H^{0}\left(K_{X}\right),\left(L^{\prime}, \psi^{\prime}\right) \oplus\left(L^{\prime}, \psi^{\prime}\right)$ is not isomorphic to any Higgs subbundle of $\left.\left(\mathcal{E}, \varphi_{\mathcal{E}}\right)\right|_{X \times t}$.

(2) $\left.\left.\left(\mathcal{E}, \varphi_{\mathcal{E}}\right)\right|_{X \times\left(\widetilde{U}_{l}-D_{l}\right)} \cong\left(\left(\widetilde{\mathcal{F}}, \varphi_{\widetilde{\mathcal{F}}}\right) \oplus\left(\widetilde{\mathcal{F}}, \varphi_{\widetilde{\mathcal{F}}}\right)\right)\right|_{X \times\left(\widetilde{U}_{l}-D_{l}\right)}$ and there is an open dense subset of $\widetilde{U}_{l}$ where $\operatorname{End}\left(\left.\left(\mathcal{E}, \varphi_{\mathcal{E}}\right)\right|_{X \times t}\right)$ is a specialization of $M(2)$. 
(3) with respect to the action of $\mathbb{C}^{\times}$on $\widetilde{\mathcal{N}}_{l}-D_{l}$, if $t_{1}, t_{2} \in \widetilde{U}_{l}-D_{l}$ lie in the same orbit, then $\left.\left.\left(\mathcal{E}, \varphi_{\mathcal{E}}\right)\right|_{X \times t_{1}} \cong\left(\mathcal{E}, \varphi_{\mathcal{E}}\right)\right|_{X \times t_{2}}$.

Proof. $\mathcal{E}$ is locally free of rank 4 because $D_{l}$ is a smooth divisor in $\widetilde{U}_{l}$. Let $(a, b, c, d, e, f) \in$ $\mathcal{N}_{l}=H^{0}\left(K_{X}\right) \oplus H^{1}\left(\mathcal{O}_{X}\right) \oplus \bar{\Psi}^{-1}(0) \subset H^{0}\left(K_{X}\right) \oplus H^{1}\left(\mathcal{O}_{X}\right) \oplus\left[H^{0}\left(L^{-2} K_{X}\right) \oplus H^{0}\left(L^{2} K_{X}\right)\right] \oplus$ $\left[H^{1}\left(L^{2}\right) \oplus H^{1}\left(L^{-2}\right)\right]$. Since the weights of the $\mathbb{C}^{\times}$-action are $0,0,-2,2,2,-2$ respectively, we can see that $\left.\left(\mathcal{F}, \varphi_{\mathcal{F}}\right)\right|_{X \times(a, b, c, d, e, f)}$ is stable if and only if $(d, e) \neq(0,0)$ and $(c, f) \neq$ $(0,0)$.

For the proofs of (2) and (3), we can follow the proofs of [12, Lemma 4.2-(2),(3)]. We have only to prove (1). For $t \in \widetilde{U}_{l}-D_{l}$, it is obvious. Let $t$ be the point in $D_{l}$ represented by a line $C=(a, b, z c, z d, z e, z f)$ parametrized by $z \in \mathbb{C}$ for $(a, b) \in H^{0}\left(K_{X}\right) \oplus H^{1}\left(\mathcal{O}_{X}\right)$, $(0,0) \neq(d, e) \in H^{0}\left(L^{2} K_{X}\right) \oplus H^{1}\left(L^{2}\right)$ and $(0,0) \neq(c, f) \in H^{0}\left(L^{-2} K_{X}\right) \oplus H^{1}\left(L^{-2}\right)$. Let $C_{0}=C \cap U_{l}$. Restricting $U_{l}$ sufficiently, we can take an open covering $\left\{V_{i}\right\}$ of $X$ such that $\left.\mathcal{F}\right|_{V_{i} \times C_{0}}$ are trivial. We fix a trivialization for each $i$. Let $\left(L_{a b}, \psi_{a b}\right)=\left.(\mathcal{L}, \psi)\right|_{X \times(a, b, 0,0,0,0)}$. Since $\left.\left(\mathcal{F}, \varphi_{\mathcal{F}}\right)\right|_{X \times 0} \cong\left(L_{a b}, \psi_{a b}\right) \oplus\left(L_{a b}^{-1},-\psi_{a b}\right)$, the transition matrices are of the form

$$
\left(\begin{array}{cc}
\lambda_{i j} & z \alpha_{i j} \\
z \beta_{i j} & \lambda_{i j}^{-1}
\end{array}\right)
$$

where $\left.\lambda_{i j}\right|_{z=0}$ is the transition for $\left.L_{a b} \cdot \varphi_{\mathcal{F}}\right|_{V_{i} \times C_{0}}$ are of the form

$$
\left(\begin{array}{cc}
p_{i} & z q_{i} \\
z r_{i} & -p_{i}
\end{array}\right)
$$

which satisfies

$$
\left(\begin{array}{cc}
\left.p_{j}\right|_{V_{i j} \times C_{0}} & \left.z q_{j}\right|_{V_{i j} \times C_{0}} \\
\left.z r_{j}\right|_{V_{i j} \times C_{0}} & -\left.p_{j}\right|_{V_{i j} \times C_{0}}
\end{array}\right)\left(\begin{array}{cc}
\lambda_{i j} & z \alpha_{i j} \\
z \beta_{i j} & \lambda_{i j}^{-1}
\end{array}\right)=\left(\begin{array}{cc}
\lambda_{i j} & z \alpha_{i j} \\
z \beta_{i j} & \lambda_{i j}^{-1}
\end{array}\right)\left(\begin{array}{cc}
\left.p_{i}\right|_{V_{i j} \times C_{0}} & \left.z q_{i}\right|_{V_{i j} \times C_{0}} \\
\left.z r_{i}\right|_{V_{i j} \times C_{0}} & -\left.p_{i}\right|_{V_{i j} \times C_{0}}
\end{array}\right)
$$

where $\left.p_{i}\right|_{z=0}=\left.\psi_{a b}\right|_{V_{i}}$ and $V_{i j}=V_{i} \cap V_{j}$. The cocycle condition of 7.1 implies that $\left\{\left.\lambda_{i j} \alpha_{i j}\right|_{z=0}\right\}$ and $\left\{\left.\lambda_{i j}^{-1} \beta_{i j}\right|_{z=0}\right\}$ are cocycles in $H^{1}\left(L_{a b}^{2}\right)$ and $H^{1}\left(L_{a b}^{-2}\right)$ respectively. Since (7.2) are cocycles, $\left\{\left.q_{i}\right|_{z=0}\right\}$ and $\left\{\left.r_{i}\right|_{z=0}\right\}$ are cocycles in $H^{0}\left(L_{a b}^{2} K_{X}\right)$ and $H^{0}\left(L_{a b}^{-2} K_{X}\right)$ respectively. Since $\left.\left(\mathcal{F}, \varphi_{\mathcal{F}}\right)\right|_{X \times(a, b, z c, z d, z e, z f)}$ is stable for $z \neq 0,\left\{\left(\left.\lambda_{i j} \alpha_{i j}\right|_{z=0},\left.q_{i}\right|_{z=0}\right)\right\}$ and $\left\{\left(\left.\lambda_{i j}^{-1} \beta_{i j}\right|_{z=0},\left.r_{i}\right|_{z=0}\right)\right\}$ are all nonzero.

Let $\left(\mathcal{F}^{\prime}, \varphi_{\mathcal{F}^{\prime}}\right)$ be the kernel of $\left.\left.\left(\mathcal{F}, \varphi_{\mathcal{F}}\right)\right|_{X \times C_{0}} \rightarrow\left(\mathcal{F}, \varphi_{\mathcal{F}}\right)\right|_{X \times 0} \cong\left(L_{a b}, \psi_{a b}\right) \oplus\left(L_{a b}^{-1},-\psi_{a b}\right)$ $\rightarrow\left(L_{a b}, \psi_{a b}\right)$ and let $\left(\mathcal{F}^{\prime \prime}, \varphi_{\mathcal{F}^{\prime \prime}}\right)$ be the kernel of $\left.\left.\left(\mathcal{F}, \varphi_{\mathcal{F}}\right)\right|_{X \times C_{0}} \rightarrow\left(\mathcal{F}, \varphi_{\mathcal{F}}\right)\right|_{X \times 0} \cong\left(L_{a b}, \psi_{a b}\right)$ $\oplus\left(L_{a b}^{-1},-\psi_{a b}\right) \rightarrow\left(L_{a b}^{-1},-\psi_{a b}\right)$. Let $\left(F^{\prime}, \phi_{F^{\prime}}\right)=\left.\left(\mathcal{F}^{\prime}, \varphi_{\mathcal{F}^{\prime}}\right)\right|_{X \times 0}$ and $\left(F^{\prime \prime}, \phi_{F^{\prime \prime}}\right)=\left(\mathcal{F}^{\prime \prime}\right.$, $\left.\varphi_{\mathcal{F}^{\prime \prime}}\right)\left.\right|_{X \times 0}$. Then

$$
\left.\left(\widetilde{\mathcal{F}}^{\prime}, \varphi_{\widetilde{\mathcal{F}}^{\prime}}\right)\right|_{X \times t} \cong\left(F^{\prime}, \phi_{F^{\prime}}\right) \quad \text { and }\left.\quad\left(\widetilde{\mathcal{F}}^{\prime \prime}, \varphi_{\widetilde{\mathcal{F}}^{\prime \prime}}\right)\right|_{X \times t} \cong\left(F^{\prime \prime}, \phi_{F^{\prime \prime}}\right)
$$


Note that any section of $\mathcal{F}^{\prime}$ over $V_{i} \times C_{0}$ is of the form $\left(z s_{1}, s_{2}\right)$. Since

$$
\left(\begin{array}{c}
s_{1} \\
s_{2}
\end{array}\right) \leftrightarrow\left(\begin{array}{c}
z s_{1} \\
s_{2}
\end{array}\right) \mapsto\left(\begin{array}{cc}
\lambda_{i j} & z \alpha_{i j} \\
z \beta_{i j} & \lambda_{i j}^{-1}
\end{array}\right)\left(\begin{array}{c}
z s_{1} \\
s_{2}
\end{array}\right)=\left(\begin{array}{c}
z\left(\lambda_{i j} s_{1}+\alpha_{i j} s_{2}\right) \\
z^{2} \beta_{i j} s_{1}+\lambda_{i j}^{-1} s_{2}
\end{array}\right) \leftrightarrow\left(\begin{array}{c}
\lambda_{i j} s_{1}+\alpha_{i j} s_{2} \\
z^{2} \beta_{i j} s_{1}+\lambda_{i j}^{-1} s_{2}
\end{array}\right),
$$

the transition for $\mathcal{F}^{\prime}$ is

$$
\left(\begin{array}{cc}
\lambda_{i j} & \alpha_{i j} \\
z^{2} \beta_{i j} & \lambda_{i j}^{-1}
\end{array}\right)
$$

Since

$$
\left(\begin{array}{c}
s_{1} \\
s_{2}
\end{array}\right) \leftrightarrow\left(\begin{array}{c}
z s_{1} \\
s_{2}
\end{array}\right) \mapsto\left(\begin{array}{cc}
p_{i} & z q_{i} \\
z r_{i} & -p_{i}
\end{array}\right)\left(\begin{array}{c}
z s_{1} \\
s_{2}
\end{array}\right)=\left(\begin{array}{c}
z\left(p_{i} s_{1}+q_{i} s_{2}\right) \\
z^{2} r_{i} s_{1}-p_{i} s_{2}
\end{array}\right) \leftrightarrow\left(\begin{array}{c}
p_{i} s_{1}+q_{i} s_{2} \\
z^{2} r_{i} s_{1}-p_{i} s_{2}
\end{array}\right)
$$

$\left.\varphi_{\mathcal{F}^{\prime}}\right|_{V_{i} \times C_{0}}$ is

$$
\left(\begin{array}{cc}
p_{i} & q_{i} \\
z^{2} r_{i} & -p_{i}
\end{array}\right)
$$

By (7.3),

$$
\left(\begin{array}{cc}
\left.p_{j}\right|_{V_{i j} \times C_{0}} & \left.q_{j}\right|_{V_{i j} \times C_{0}} \\
\left.z^{2} r_{j}\right|_{V_{i j} \times C_{0}} & -\left.p_{j}\right|_{V_{i j} \times C_{0}}
\end{array}\right)\left(\begin{array}{cc}
\lambda_{i j} & \alpha_{i j} \\
z^{2} \beta_{i j} & \lambda_{i j}^{-1}
\end{array}\right)=\left(\begin{array}{cc}
\lambda_{i j} & \alpha_{i j} \\
z^{2} \beta_{i j} & \lambda_{i j}^{-1}
\end{array}\right)\left(\begin{array}{cc}
\left.p_{i}\right|_{V_{i j} \times C_{0}} & \left.q_{i}\right|_{V_{i j} \times C_{0}} \\
\left.z^{2} r_{i}\right|_{V_{i j} \times C_{0}} & -\left.p_{i}\right|_{V_{i j} \times C_{0}}
\end{array}\right) .
$$

Thus $\left(F^{\prime}, \phi_{F^{\prime}}\right)$ fits into a commutative diagram

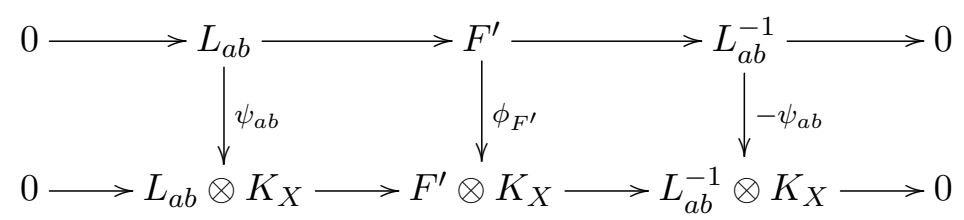

where each row is a short exact sequence and the extension class is $\left\{\left(\left.\lambda_{i j} \alpha_{i j}\right|_{z=0},\left.q_{i}\right|_{z=0}\right)\right\}$, that is, $\left(F^{\prime}, \phi_{F^{\prime}}\right)$ is a nonsplit extension of $\left(L_{a b}^{-1},-\psi_{a b}\right)$ by $\left(L_{a b}, \psi_{a b}\right)$. Similarly $\left(F^{\prime \prime}, \phi_{F^{\prime \prime}}\right)$ is also a nonsplit extension of $\left(L_{a b}, \psi_{a b}\right)$ by $\left(L_{a b}^{-1},-\psi_{a b}\right)$ whose extension class is $\left\{\left(\left.\lambda_{i j}^{-1} \beta_{i j}\right|_{z=0}\right.\right.$, $\left.\left.\left.r_{i}\right|_{z=0}\right)\right\}$. Hence we conclude that $\left.\left(\mathcal{E}, \phi_{\mathcal{E}}\right)\right|_{X \times t}=\left(F^{\prime}, \phi_{F^{\prime}}\right) \oplus\left(F^{\prime \prime}, \phi_{F^{\prime \prime}}\right)$ does not contain a Higgs subbundle isomorphic to $\left(L^{\prime}, \psi^{\prime}\right) \oplus\left(L^{\prime}, \psi^{\prime}\right)$ for any $L^{\prime} \in \operatorname{Pic}^{0}(X)$ and $\psi^{\prime} \in H^{0}\left(K_{X}\right)$.

It follows from Proposition 7.1 and Luna's slice theorem that $\rho^{\prime}$ extends continuously to a neighborhood of the points in $\mathbf{K}$ lying over $l$. Since there is at most one continuous extension, the extensions for various points $l$ in the middle stratum $T^{*} J / \mathbb{Z}_{2}-\mathbb{Z}_{2}^{2 g}$ are compatible. Thus $\rho^{\prime}$ is extended to all the points in $\mathbf{K}$ except those over the deepest strata $\mathbb{Z}_{2}^{2 g}$. 


\subsubsection{Points over the deepest strata}

We next extend $\rho^{\prime}$ to the points over the deepest strata $\mathbb{Z}_{2}^{2 g}$. We may consider only the points in $\mathbf{K}$ over $0=[(\mathcal{O}, 0) \oplus(\mathcal{O}, 0)] \in \mathbb{Z}_{2}^{2 g}$. Let $\mathcal{N}=\bar{\Upsilon}^{-1}(0)$. Since it follows from Theorem 4.1 that a neighborhood of $[(\mathcal{O}, 0) \oplus(\mathcal{O}, 0)]$ in $\mathbf{M}_{2}$ is analytically equivalent to a neighborhood of the vertex $\overline{0}$ in the cone $\mathcal{N} / / \mathrm{SL}(2)$, a neighborhood of the preimage of $[(\mathcal{O}, 0) \oplus(\mathcal{O}, 0)]$ in $\mathbf{K}$ is bihomolorphic to an open subset of the desingularization $\tilde{\mathcal{N}} / / \operatorname{SL}(2)$, obtained by three blow-ups from $\mathcal{N} / / \mathrm{SL}(2)$, which will be constructed below. Then it is enough to construct a holomorphic map from a neighborhood $\widetilde{V}$ of the preimage of $\overline{0}$ in $\widetilde{\mathcal{N}} / / \mathrm{SL}(2)$ to $\mathbf{S}$.

Let $\Sigma=\operatorname{SL}(2)\left\{\left[H^{0}\left(K_{X}\right) \oplus H^{1}\left(\mathcal{O}_{X}\right)\right] \otimes\left(\begin{array}{cc}1 & 0 \\ 0 & -1\end{array}\right)\right\}$. Let $\pi_{1}: \mathcal{N}_{1} \rightarrow \mathcal{N}$ be the blow-up of $\mathcal{N}$ at 0 with the exceptional divisor $\mathcal{D}_{1}^{(1)}$. Let

$$
\Delta=\operatorname{SL}(2) \mathbb{P}\left\{\left(\begin{array}{ll}
0 & b \\
c & 0
\end{array}\right) \mid b, c \in H^{0}\left(K_{X}\right) \oplus H^{1}\left(\mathcal{O}_{X}\right),\langle b, c\rangle=0\right\}
$$

as a subset of $\mathcal{D}_{1}^{(1)}$, where $\langle\cdot, \cdot\rangle$ is the perfect pairing on $H^{0}\left(K_{X}\right) \oplus H^{1}\left(\mathcal{O}_{X}\right)$. Let $\widetilde{\Sigma}$ be the strict transform of $\Sigma$ in $\mathcal{N}_{1}$. Then the singular locus of $\mathcal{N}_{1} / / \operatorname{SL}(2)$ is the quotient $\Delta \cap \widetilde{\Sigma} / / \operatorname{SL}(2)$. Note that

$$
\Delta \cap \widetilde{\Sigma}=\operatorname{SL}(2) \mathbb{P}\left\{\left(H^{0}\left(K_{X}\right) \oplus H^{1}\left(\mathcal{O}_{X}\right)\right) \otimes\left(\begin{array}{cc}
1 & 0 \\
0 & -1
\end{array}\right)\right\}=\mathcal{D}_{1}^{(1)} \cap \widetilde{\Sigma} .
$$

Let $\pi_{2}: \mathcal{N}_{2} \rightarrow \mathcal{N}_{1}$ be the blow-up of $\mathcal{N}_{1}$ along $\widetilde{\Sigma}$ with the exceptional divisor $\mathcal{D}_{2}^{(2)}$. Let $\mathcal{D}_{2}^{(1)}$ be the strict transform of $\mathcal{D}_{1}^{(1)}$. The singular locus of $\mathcal{N}_{2} / / \operatorname{SL}(2)$ is the quotient $\widetilde{\Delta} / / \operatorname{SL}(2)$, where $\widetilde{\Delta}$ is the strict transform of $\Delta$.

Let $\pi_{3}: \widetilde{\mathcal{N}}=\mathcal{N}_{3} \rightarrow \mathcal{N}_{2}$ be the blow-up of $\mathcal{N}_{2}$ along $\widetilde{\Delta}$ with the exceptional divisor $\widetilde{\mathcal{D}}^{(3)}=\mathcal{D}_{3}^{(3)}$ and let $\widetilde{\mathcal{D}}^{(1)}=\mathcal{D}_{3}^{(1)}, \widetilde{\mathcal{D}}^{(2)}=\mathcal{D}_{3}^{(2)}$ be the strict transforms of $\mathcal{D}_{2}^{(1)}$ and $\mathcal{D}_{2}^{(2)}$ respectively. Let $\pi: \widetilde{\mathcal{N}} \rightarrow \mathcal{N}$ be the composition $\pi_{1} \circ \pi_{2} \circ \pi_{3}$. Let $D_{i}^{(j)}$ be the quotient of $\mathcal{D}_{i}^{(j)}$ in $\mathcal{N}_{i} / / \mathrm{SL}(2)$ for $1 \leq i \leq 3$ and $1 \leq j \leq i$.

It follows from a similar argument as in the case of the middle stratum that the universal family over $X \times \mathbf{R}$ induces a holomorphic family $\left(\mathcal{F}, \varphi_{\mathcal{F}}\right)$ of rank 2 semistable Higgs bundles over $X$ parametrized by an open neighborhood $U$ of 0 in $\mathcal{N}^{s s}$. Let $V$ be the image of $U$ in $\mathcal{N} / / \operatorname{SL}(2)$. Then $V$ is an open neighborhood of $\overline{0}$ in $\mathcal{N} / / \mathrm{SL}(2)$. Let $U_{1}=\pi_{1}^{-1}(U) \cap \mathcal{N}_{1}^{s s}$ and $V_{1}$ be the image of $U_{1}$ in $\mathcal{N}_{1} / / \operatorname{SL}(2)$. It follows from the commutative diagram

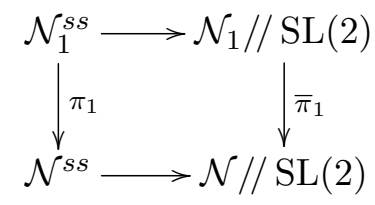


that we have $V_{1}=\bar{\pi}_{1}^{-1}(V)$.

Let $U_{2}=\pi_{2}^{-1}\left(U_{1}\right) \cap \mathcal{N}_{2}^{s s}$ and $V_{2}$ be the image of $U_{2}$ in $\mathcal{N}_{2} / / \operatorname{SL}(2)$. Then $V_{2}=\bar{\pi}_{2}^{-1}\left(V_{1}\right)$ where $\bar{\pi}_{2}: \mathcal{N}_{2} / / \operatorname{SL}(2) \rightarrow \mathcal{N}_{1} / / \operatorname{SL}(2)$. Let $\widetilde{U}=\pi_{3}^{-1}\left(U_{2}\right) \cap \tilde{\mathcal{N}}^{s s}$ and $\widetilde{V}$ be the image of $\widetilde{U}$ in $\widetilde{\mathcal{N}} / / \mathrm{SL}(2)$. Then $\widetilde{V}$ is smooth with simple normal crossing divisors $\widetilde{D}^{(1)}, \widetilde{D}^{(2)}, \widetilde{D}^{(3)}$ where $\widetilde{D}^{(j)}=\widetilde{D}_{3}^{(j)}$ and $\widetilde{D}^{(2)} \cap \widetilde{V}$ is denoted again by $\widetilde{D}^{(2)}$.

$\rho^{\prime}$ was already extended to the points over the middle stratum, so we have a holomorphic map $\rho^{\prime}: \widetilde{V}-\left(\widetilde{D}^{(1)} \cap \widetilde{D}^{(3)}\right) \rightarrow \mathbf{S}$. Now $\rho^{\prime}$ must be extended to $\rho: \widetilde{V} \rightarrow \mathbf{S}$.

\subsubsection{Points over $\widetilde{D}^{(1)}-\left(\widetilde{D}^{(2)} \cup \widetilde{D}^{(3)}\right)$}

In this subsection, we will extend $\rho^{\prime}$ to points in $\widetilde{V}$ lying over the quotient of $\mathcal{D}_{1}^{(1)}-\Delta$.

We want to modify the pull-back of $\left(\mathcal{F}, \varphi_{\mathcal{F}}\right) \oplus\left(\mathcal{F}, \varphi_{\mathcal{F}}\right)$ to $U_{1}-\Delta \cup \widetilde{\Sigma}$ so that $\rho^{\prime}$ is extended to a holomorphic map near the quotient of $\mathcal{D}_{1}^{(1)}-\Delta$ by Proposition 7.1.

Let $\left(\mathcal{F}_{1}, \varphi_{\mathcal{F}_{1}}\right)$ be the pull-back of $\left(\mathcal{F}, \varphi_{\mathcal{F}}\right)$ to $X \times U_{1}$ via $1_{X} \times \pi_{1}$. Since $\left.\left(\mathcal{F}, \varphi_{\mathcal{F}}\right)\right|_{X \times 0}$ is trivial, we have $\left.\left(\mathcal{F}_{1}, \varphi_{\mathcal{F}_{1}}\right)\right|_{X \times \mathcal{D}_{1}^{(1)}} \cong(\mathcal{O}, 0) \oplus(\mathcal{O}, 0)$. Let $\left(\mathcal{F}_{1}^{\prime}, \varphi_{\mathcal{F}_{1}^{\prime}}\right)$ (resp. $\left.\left(\mathcal{F}_{1}^{\prime \prime}, \varphi_{\mathcal{F}_{1}^{\prime \prime}}\right)\right)$ be the kernel of

$$
\left.\left(\mathcal{F}_{1}, \varphi_{\mathcal{F}_{1}}\right) \rightarrow\left(\mathcal{F}_{1}, \varphi_{\mathcal{F}_{1}}\right)\right|_{X \times \mathcal{D}_{1}^{(1)}} \cong\left(\mathcal{O}_{X \times \mathcal{D}_{1}^{(1)}}, 0\right) \oplus\left(\mathcal{O}_{X \times \mathcal{D}_{1}^{(1)}}, 0\right) \rightarrow\left(\mathcal{O}_{X \times \mathcal{D}_{1}^{(1)}}, 0\right)
$$

where the second arrow is the projection onto the first component (resp. the second component). A computation of transition matrices as in the proof of Lemma 7.3 tells us that $\left.\left(\mathcal{F}_{1}^{\prime}, \varphi_{\mathcal{F}_{1}^{\prime}}\right)\right|_{X \times t_{1}}$ and $\left.\left(\mathcal{F}_{1}^{\prime \prime}, \varphi_{\mathcal{F}_{1}^{\prime \prime}}\right)\right|_{X \times t_{1}}$ are nonsplit extensions of $(\mathcal{O}, 0)$ by $(\mathcal{O}, 0)$ for $t_{1}=\left(\left(\begin{array}{cc}a & b \\ c & -a\end{array}\right),\left(\begin{array}{cc}d & e \\ f & -d\end{array}\right)\right) \in \mathbb{P} \mathcal{N}=\mathcal{D}_{1}^{(1)}$ with $(b, e) \neq(0,0)$ and $(c, f) \neq(0,0)$ in $\mathbf{H}^{1} \operatorname{Hom}((\mathcal{O}, 0),(\mathcal{O}, 0))=H^{0}\left(K_{X}\right) \oplus H^{1}\left(\mathcal{O}_{X}\right)$.

Assume that $t_{1} \in \mathcal{D}_{1}^{(1)}-\Delta$. Then $(a, d),(b, e),(c, f)$ are linearly independent. In particular, $(a, d),(b, e),(c, f)$ are all nonzero, which implies that $\left.\left(\mathcal{F}_{1}^{\prime}, \varphi_{\mathcal{F}_{1}^{\prime}}\right)\right|_{X \times t_{1}}$ and $\left.\left(\mathcal{F}_{1}^{\prime \prime}, \varphi_{\mathcal{F}_{1}^{\prime \prime}}\right)\right|_{X \times t_{1}}$ are nonsplit extensions of $(\mathcal{O}, 0)$ by $(\mathcal{O}, 0)$ with extension classes $(b, e)$ and $(c, f)$ respectively.

The inclusion $\left(\mathcal{F}_{1}^{\prime}, \varphi_{\mathcal{F}_{1}^{\prime}}\right) \hookrightarrow\left(\mathcal{F}_{1}, \varphi_{\mathcal{F}_{1}}\right)$ induces a homomorphism $\left.\left(\mathcal{F}_{1}^{\prime}, \varphi_{\mathcal{F}_{1}^{\prime}}\right)\right|_{X \times \mathcal{D}_{1}^{(1)}} \rightarrow$ $\left.\left(\mathcal{F}_{1}, \varphi_{\mathcal{F}_{1}}\right)\right|_{X \times \mathcal{D}_{1}^{(1)}} \cong(\mathcal{O}, 0) \oplus(\mathcal{O}, 0)$ whose image is the second factor $(\mathcal{O}, 0)$ and the kernel of this homomorphism is $(\mathcal{O}, 0)$. Similarly, $\left(\mathcal{O}_{X \times \mathcal{D}_{1}^{(1)}}, 0\right)$ is a Higgs subbundle of $\left(\mathcal{F}_{1}^{\prime \prime}, \varphi_{\mathcal{F}_{1}^{\prime \prime}}\right)$. Then we have a diagonal embedding of $\left(\mathcal{O}_{X \times \mathcal{D}_{1}^{(1)}}, 0\right)$ into $\left.\left(\mathcal{F}_{1}^{\prime}, \varphi_{\mathcal{F}_{1}^{\prime}}\right) \oplus\left(\mathcal{F}_{1}^{\prime \prime}, \varphi_{\mathcal{F}_{1}^{\prime \prime}}\right)\right|_{X \times \mathcal{D}_{1}^{(1)}}$. Let $\left(\mathcal{E}_{1}, \varphi_{\mathcal{E}_{1}}\right)$ be the kernel of

$$
\begin{aligned}
\left(\mathcal{F}_{1}^{\prime} \oplus \mathcal{F}_{1}^{\prime \prime}, \varphi_{\mathcal{F}_{1}^{\prime} \oplus \mathcal{F}_{1}^{\prime \prime}}\right) & \rightarrow\left(\left.\mathcal{F}_{1}^{\prime} \oplus \mathcal{F}_{1}^{\prime \prime}\right|_{X \times \mathcal{D}_{1}^{(1)}}, \varphi_{\left.\mathcal{F}_{1}^{\prime} \oplus \mathcal{F}_{1}^{\prime \prime}\right|_{X \times \mathcal{D}_{1}^{(1)}}}\right) \\
& \rightarrow\left(\left.\mathcal{F}_{1}^{\prime} \oplus \mathcal{F}_{1}^{\prime \prime}\right|_{X \times \mathcal{D}_{1}^{(1)}} / \mathcal{O}_{X \times \mathcal{D}_{1}^{(1)}}, \varphi_{\left(\left.\mathcal{F}_{1}^{\prime} \oplus \mathcal{F}_{1}^{\prime \prime}\right|_{X \times \mathcal{D}_{1}^{(1)}} / \mathcal{O}_{\left.X \times \mathcal{D}_{1}^{(1)}\right)}\right)} .\right.
\end{aligned}
$$

Adapting the argument of $\left[12\right.$, Section 4.3], we can see that the transition for $\left.\mathcal{E}_{1}\right|_{X \times t_{1}}$ 
is

$$
\left(\begin{array}{cccc}
1 & 0 & 0 & 0 \\
0 & 1 & 0 & 0 \\
0 & 0 & 1 & 0 \\
-\left.2 \alpha_{i j}\right|_{z=0} & -\left.\beta_{i j}\right|_{z=0} & \left.\gamma_{i j}\right|_{z=0} & 1
\end{array}\right)
$$

and $\left.\varphi_{\mathcal{E}_{1}}\right|_{X \times t_{1}}$ is locally

$$
\left(\begin{array}{cccc}
0 & 0 & 0 & 0 \\
0 & 0 & 0 & 0 \\
0 & 0 & 0 & 0 \\
-\left.2 p_{i}\right|_{z=0} & -\left.q_{i}\right|_{z=0} & \left.r_{i}\right|_{z=0} & 0
\end{array}\right)
$$

where the cocycles $\left\{\left(\left.\alpha_{i j}\right|_{z=0},\left.p_{i}\right|_{z=0}\right)\right\},\left\{\left(\left.\beta_{i j}\right|_{z=0},\left.q_{i}\right|_{z=0}\right)\right\},\left\{\left(\left.\gamma_{i j}\right|_{z=0},\left.r_{i}\right|_{z=0}\right)\right\}$ represent the classes $(a, d),(b, e),(c, f) \in H^{1}\left(\mathcal{O}_{X}\right) \oplus H^{0}\left(K_{X}\right)$ respectively.

It is easy to check that

$$
\begin{aligned}
& \left(\begin{array}{rrrr}
0 & 0 & 0 & 0 \\
0 & 0 & 0 & 0 \\
0 & 0 & 0 & 0 \\
-\left.2 p_{j}\right|_{z=0} & -\left.q_{j}\right|_{z=0} & \left.r_{j}\right|_{z=0} & 0
\end{array}\right)\left(\begin{array}{cccc}
1 & 0 & 0 & 0 \\
0 & 1 & 0 & 0 \\
0 & 0 & 1 & 0 \\
-\left.2 \alpha_{i j}\right|_{z=0} & -\left.\beta_{i j}\right|_{z=0} & \left.\gamma_{i j}\right|_{z=0} & 1
\end{array}\right) \\
& =\left(\begin{array}{cccc}
1 & 0 & 0 & 0 \\
0 & 1 & 0 & 0 \\
0 & 0 & 1 & 0 \\
-\left.2 \alpha_{i j}\right|_{z=0} & -\left.\beta_{i j}\right|_{z=0} & \left.\gamma_{i j}\right|_{z=0} & 1
\end{array}\right)\left(\begin{array}{rrrr}
0 & 0 & 0 & 0 \\
0 & 0 & 0 & 0 \\
0 & 0 & 0 & 0 \\
-\left.2 p_{i}\right|_{z=0} & -\left.q_{i}\right|_{z=0} & \left.r_{i}\right|_{z=0} & 0
\end{array}\right) .
\end{aligned}
$$

Hence we have a filtration by Higgs subbundles

$$
\left.\left(\mathcal{E}_{1}, \varphi_{\mathcal{E}_{1}}\right)\right|_{X \times t_{1}}=\left(E_{4}, \phi_{E_{4}}\right) \supset\left(E_{3}, \phi_{E_{3}}\right) \supset\left(E_{2}, \phi_{E_{2}}\right) \supset\left(E_{1}, \phi_{E_{1}}\right) \supset\left(E_{0}, \phi_{E_{0}}\right)=0
$$

such that $\left(E_{i+1} / E_{i}, \phi_{E_{i+1} / E_{i}}\right) \cong\left(\mathcal{O}_{X}, 0\right)$. Since $(c, f) \neq(0,0)$, the extension $\left(E_{2}, \phi_{E_{2}}\right)$ of $(\mathcal{O}, 0)$ by $\left(E_{1}, \phi_{E_{1}}\right) \cong(\mathcal{O}, 0)$ is nontrivial. Since $(b, e),(c, f)$ are linearly independent, an extension of $(\mathcal{O}, 0)$ by $\left(E_{2}, \phi_{E_{2}}\right)$ is parametrized by $\mathbf{H}^{1} \mathbf{H o m}\left((\mathcal{O}, 0),\left(E_{2}, \phi_{E_{2}}\right)\right)$ which fits in the exact sequence

$$
\mathbf{H}^{0} \mathbf{H o m}((\mathcal{O}, 0),(\mathcal{O}, 0)) \stackrel{(c, f)}{\longrightarrow} \mathbf{H}^{1} \mathbf{H o m}((\mathcal{O}, 0),(\mathcal{O}, 0))
$$


and $\left(E_{3}, \phi_{E_{3}}\right)$ is the image of

$$
(b, e) \in \mathbf{H}^{1}(\mathbf{H o m}((\mathcal{O}, 0),(\mathcal{O}, 0))) \cong H^{1}\left(\mathcal{O}_{X}\right) \oplus H^{0}\left(K_{X}\right)
$$

which is nonzero. Thus $\left(E_{3}, \phi_{E_{3}}\right)$ is a nonsplit extension. Similarly $\left(E_{4}, \phi_{E_{4}}\right)$ is a nonsplit extension since $(a, d),(b, e),(c, f)$ are linearly independent. Thus the condition (a) of Proposition $7.1(1)$ holds for points in $\widetilde{U}$ over $\mathcal{D}_{1}^{(1)}-\Delta$. The other conditions of Proposition 7.1 obviously hold. Therefore $\rho^{\prime}$ is extended to the points over the quotient of the points over $\mathcal{D}_{1}^{(1)}-\Delta$.

\subsubsection{Points over $\widetilde{D}^{(3)}-\widetilde{D}^{(2)}$}

Let $t_{1}=\left(\left(\begin{array}{cc}a & b \\ c & -a\end{array}\right),\left(\begin{array}{cc}d & e \\ f & -d\end{array}\right)\right) \in \Delta-\widetilde{\Sigma}$. Then $(a, d),(b, e),(c, f)$ span 2-dimensional subspace of $H^{1}\left(\mathcal{O}_{X}\right) \oplus H^{0}\left(K_{X}\right)$. The Higgs bundle $\left.\left(\mathcal{E}_{1}, \varphi_{\mathcal{E}_{1}}\right)\right|_{X \times t_{1}}$ has transition matrices and local Higgs fields of the form (7.5) and (7.6). A Higgs bundle of rank 1 over $\Delta-\widetilde{\Sigma}$ is obtained from the family of linear relations of $(a, d),(b, e),(c, f) .\left(\mathcal{L}_{1}, 0\right)$ denotes the pull-back of this Higgs bundle of rank 1 to $X \times(\Delta-\widetilde{\Sigma})$. Then there is an embedding of $\left(\mathcal{L}_{1}, 0\right) \hookrightarrow$ $\left.\left(\mathcal{E}_{1}, \varphi_{\mathcal{E}_{1}}\right)\right|_{X \times(\Delta-\widetilde{\Sigma})}$. Let $\left(\mathcal{E}_{3}, \varphi_{\mathcal{E}_{3}}\right)\left(\operatorname{resp} .\left(\mathcal{L}_{3}, 0\right)\right)$ be the pull-back of $\left(\mathcal{E}_{1}, \varphi_{\mathcal{E}_{1}}\right)\left(\operatorname{resp} .\left(\mathcal{L}_{1}, 0\right)\right)$ to $\widetilde{U}=U_{3}\left(\operatorname{resp} . \widetilde{\mathcal{D}}^{(3)}-\widetilde{\mathcal{D}}^{(2)}\right)$.

Let $\left(\widetilde{\mathcal{E}}, \varphi_{\widetilde{\mathcal{E}}}\right)$ be the kernel of

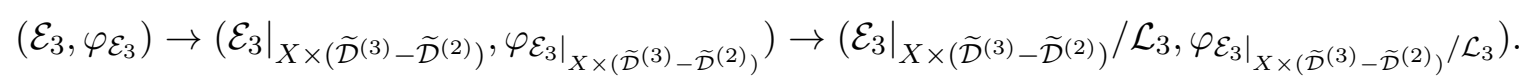

Let $t_{1}=\left(\left(\begin{array}{ll}0 & b \\ c & 0\end{array}\right),\left(\begin{array}{ll}0 & e \\ f & 0\end{array}\right)\right) \in \Delta-\widetilde{\Sigma}$ with $(b, e),(c, f)$ linearly independent for simplicity. (The general case is obtained by conjugation.) Let $t_{3} \in \widetilde{\mathcal{D}}^{(3)}-\widetilde{\mathcal{D}}^{(2)}$ be a (semi)stable point lying over $t_{1}$. A point $t_{3} \in \widetilde{\mathcal{D}}^{(3)}$ represents a normal direction to $\Delta$ at $t_{1}$. Choose a local parameter $z$ to the direction such that $z=0$ represents $t_{1}$. We make local computations as in the previous subsection 97.1 .3 .

If $t_{3}$ represents a normal direction of $\Delta$ tangent to $\widetilde{\mathcal{D}}^{(1)}$, then the transition and local Higgs field of $\left.\left(\widetilde{\mathcal{E}}, \varphi_{\widetilde{\mathcal{E}}}\right)\right|_{X \times t_{3}}$ are of the form

$$
\left(\begin{array}{ccrr}
1 & 0 & 0 & 0 \\
0 & 1 & 0 & 0 \\
0 & 0 & 1 & 0 \\
-\left.2 \delta_{i j}\right|_{z=0} & -\left.\beta_{i j}\right|_{z=0} & \left.\gamma_{i j}\right|_{z=0} & 1
\end{array}\right)
$$

and

$$
\left(\begin{array}{cccc}
0 & 0 & 0 & 0 \\
0 & 0 & 0 & 0 \\
0 & 0 & 0 & 0 \\
-\left.2 s_{i}\right|_{z=0} & -\left.q_{i}\right|_{z=0} & \left.r_{i}\right|_{z=0} & 0
\end{array}\right)
$$


where $\left\{\left(\delta_{i j}, s_{i}\right)\right\}$ are some cocycles which give us a nonzero class $(g, h) \in H^{0}\left(K_{X}\right) \oplus H^{1}\left(\mathcal{O}_{X}\right)$ at $z=0$ such that $(g, h),(b, e),(c, f)$ are linearly independent. The condition (1) of Proposition 7.1 holds because the Higgs bundle has a filtration by Higgs subbundles as in (7.7).

If $t_{3}$ represents the direction normal to $\widetilde{\mathcal{D}}^{(1)}$, then the transition and local Higgs field for $\left.\left(\widetilde{\mathcal{E}}, \varphi_{\widetilde{\mathcal{E}}}\right)\right|_{X \times t_{3}}$ are of the form

$$
\left(\begin{array}{ccrr}
1 & 0 & 0 & 0 \\
\left.\gamma_{i j}\right|_{z=0} & 1 & 0 & 0 \\
\left.\beta_{i j}\right|_{z=0} & 0 & 1 & 0 \\
-\left.2 \delta_{i j}\right|_{z=0} & -\left.\beta_{i j}\right|_{z=0} & \left.\gamma_{i j}\right|_{z=0} & 1
\end{array}\right)
$$

and

$$
\left(\begin{array}{cccc}
0 & 0 & 0 & 0 \\
\left.r_{i}\right|_{z=0} & 0 & 0 & 0 \\
\left.q_{i}\right|_{z=0} & 0 & 0 & 0 \\
-\left.2 s_{i}\right|_{z=0} & -\left.q_{i}\right|_{z=0} & \left.r_{i}\right|_{z=0} & 0
\end{array}\right) .
$$

Since the Higgs bundle has a filtration by Higgs subbundles as in $(7.7),\left.\left(\widetilde{\mathcal{E}}, \varphi_{\widetilde{\mathcal{E}}}\right)\right|_{X \times t_{3}}$ satisfies the condition (1) of Proposition 7.1.

Since the other conditions of Proposition 7.1 automatically hold on the stable part of $U, \rho^{\prime}$ extends to the quotient of $\widetilde{U}-\widetilde{\mathcal{D}}^{(2)}$.

$$
\text { 7.1.5. Points over } \widetilde{D}^{(2)} \cap\left(\widetilde{D}^{(1)} \cup \widetilde{D}^{(3)}\right)
$$

In this subsection $\rho^{\prime}$ will be finally extended to $\mathbf{K}$ and then we complete the proof of Proposition 7.2. The slice theorem gives us a map $\widetilde{V} \rightarrow \mathbf{K}$ which is biholomorphic onto a neighborhood of the preimage of $[(\mathcal{O}, 0) \oplus(\mathcal{O}, 0)]$. So it is sufficient to construct a holomorphic map $\widetilde{V} \rightarrow \mathbf{S}$.

There is a commutative diagram

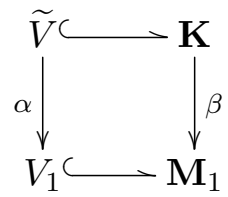

where $\mathbf{M}_{1}$ is the first blow-up in the Kirwan's process and the vertical maps are blow-ups. A holomorphic map

$$
\nu: \widetilde{V}-\alpha^{-1}(\Delta \cap \widetilde{\Sigma} / / \mathrm{SL}(2)) \rightarrow \mathbf{S}
$$


was already constructed.

Let $x$ be any point in $\Delta \cap \widetilde{\Sigma} / / \operatorname{SL}(2)$. It follows from $(7.4)$ that $x$ is represented by the orbit of $\left[\begin{array}{cc}a^{0}+p^{0} & 0 \\ 0 & -a^{0}-p^{0}\end{array}\right]$ for some $a^{0} \in H^{1}\left(\mathcal{O}_{X}\right)$ and $p^{0} \in H^{0}\left(K_{X}\right)$. The normal space $Y$ to this orbit is isomorphic to $\mathbb{C}^{2 g} \times \bar{\Psi}^{-1}(0)$ where $\mathbb{C}^{2 g}$ is the tangent space of the blow-up $H^{1}\left(\mathcal{O}_{X} \widehat{\oplus \oplus H^{0}}\left(K_{X}\right)=b l_{0}\left(H^{1}\left(\mathcal{O}_{X}\right) \oplus H^{0}\left(K_{X}\right)\right)\right.$.

For a neighborhood $Y_{1}$ of 0 in $Y$ which is holomorphically embedded into $U_{1},\left(\mathcal{F}_{1}\right.$, $\left.\varphi_{\mathcal{F}_{1}}\right)\left.\right|_{X \times Y_{1}}$ has transition matrices of the form

$$
\left(\begin{array}{cc}
1+z_{1}\left(a_{i j}^{0}+a_{i j}\right) & z_{1} b_{i j} \\
z_{1} c_{i j} & 1-z_{1}\left(a_{i j}^{0}+a_{i j}\right)
\end{array}\right)
$$

and local Higgs fields of the form

$$
\left(\begin{array}{cc}
z_{1}\left(p_{i}^{0}+p_{i}\right) & z_{1} q_{i} \\
z_{1} r_{i} & -z_{1}\left(p_{i}^{0}+p_{i}\right)
\end{array}\right)
$$

where $(a, p)=\left(\left\{a_{i j}\right\},\left\{p_{i}\right\}\right),(b, q)=\left(\left\{b_{i j}\right\},\left\{q_{i}\right\}\right),(c, r)=\left(\left\{c_{i j}\right\},\left\{r_{i}\right\}\right)$ are classes in $H^{1}\left(\mathcal{O}_{X}\right) \oplus H^{0}\left(K_{X}\right)$, not parallel to $\left(a^{0}, p^{0}\right)$ if they are nonzero and $z_{1}$ is the coordinate for the normal direction of $\mathbb{P}\left(H^{1}\left(\mathcal{O}_{X}\right) \oplus H^{0}\left(K_{X}\right)\right)$ in $H^{1}\left(\mathcal{O}_{X} \widetilde{\oplus H^{0}}\left(K_{X}\right)\right.$.

Since Luna's étale slice theorem tells us that a neighborhood of the vertex of the cone $Y / / \mathbb{C}^{\times}$is analytically equivalent to a neighborhood of $x$ in $V_{1}$, the image of $\tilde{Y}$ in $\widetilde{V}$ is biholomorphic to a neighborhood of $\alpha^{-1}(x)$ where $\widetilde{Y}$ is the strict transform of $Y_{1}$ in $\widetilde{U}$.

Following the same argument as in [12,4.5], we can construct a family of rank 4 Higgs bundles on $X$ parametrized by $\widetilde{Y}$ satisfying the conditions of Proposition 7.1 . Then $\nu$ can extends to $\alpha^{-1}(x)$.

\subsection{Contractions}

Let $\left(\mathbb{C}^{2 g}, \omega\right)$ be a symplectic vector space and let $\operatorname{Gr}^{\omega}(k, 2 g)$ be the Grassmannian of $k$-dimensional subspaces of $\mathbb{C}^{2 g}$ such that the restriction of $\omega$ to the subspace is zero. Let $\mathcal{A}$ (resp. $\mathcal{B}$ ) be the tautological rank 2 (resp. rank 3) bundle over the Grassmannian $\operatorname{Gr}^{\omega}(2,2 g)$ (resp. $\left.\operatorname{Gr}^{\omega}(3,2 g)\right)$. For $B \in \operatorname{Gr}^{\omega}(3,2 g)$, let

$$
\begin{array}{r}
\mathbf{C C}(B):=\text { the closure of }\left\{(C, D) \in \mathbb{P}\left(S^{2} B\right) \times \mathbb{P}\left(S^{2} B^{\vee}\right) \mid\right. \\
C \text { and } D \text { are smooth conics dual to each other }\},
\end{array}
$$

which is called the variety of complete conics. Then we have two projections

$$
\mathbb{P}\left(S^{2} B\right)<\stackrel{\Phi_{B}}{\leftarrow} \mathbf{C C}(B) \stackrel{\Phi_{B}^{\vee}}{\longrightarrow} \mathbb{P}\left(S^{2} B^{\vee}\right)
$$


where each of $\Phi_{B}, \Phi_{\breve{B}}$ is the blow-up of each of $\mathbb{P}\left(S^{2} B\right), \mathbb{P}\left(S^{2} B^{\vee}\right)$ along the locus of rank 1 conics. Let $\mathbf{C C}(\mathcal{B})$ be the tautological family of complete conics over $\operatorname{Gr}^{\omega}(3,2 g)$. Then the restrictions of the exceptional divisors and their intersections over a point in $\mathbb{Z}_{2}^{2 g}$ can be described as follows (see also [13, Proposition 4.2]).

Proposition 7.4. (1) $\widetilde{D}^{(1)} \cong \mathbf{C C}(\mathcal{B})$, that is, $\widetilde{D}^{(1)}$ is the blow-up of $\mathbb{P}\left(S^{2} \mathcal{B}\right)$ along the locus of rank 1 conics. Hence $\widetilde{D}^{(1)}$, that is, $\widetilde{D}^{(1)}$ is a $\widehat{\mathbb{P}}^{5}$-bundle over $\operatorname{Gr}^{\omega}(3,2 g)$ where $\widehat{\mathbb{P}}^{5}$ is the blow-up of $\mathbb{P}^{5}$ (projectivization of the space of $3 \times 3$ symmetric matrices) along $\mathbb{P}^{2}$ (the locus of rank 1 matrices).

(2) $\widetilde{D}^{(3)}$ is a $\mathbb{P}^{2 g-4}$-bundle over $\mathbb{P}\left(S^{2} \mathcal{A}\right)$. Hence $\widetilde{D}^{(3)}$ is a $\mathbb{P}^{2 g-4}$-bundle over a $\mathbb{P}^{2}$-bundle over $\operatorname{Gr}^{\omega}(2,2 g)$.

(3) $\widetilde{D}^{(1)} \cap \widetilde{D}^{(3)}$ is the proper transform of $\mathbb{P}\left(S^{2} \mathcal{B}\right)_{2}$ (the locus of rank $\leq 2$ matrices) in the blow-up $\mathbf{C C}(\mathcal{B}) \rightarrow \mathbb{P}\left(S^{2} \mathcal{B}\right)$, that is, the exceptional divisor of the blow-up $\mathbf{C C}(\mathcal{B}) \rightarrow$ $\mathbb{P}\left(S^{2} \mathcal{B}^{\vee}\right)$. Hence $\widetilde{D}^{(1)} \cap \widetilde{D}^{(3)}$ is a $\mathbb{P}^{2}$-bundle over a $\mathbb{P}^{2}$-bundle over $\operatorname{Gr}^{\omega}(3,2 g)$.

(4) $\widetilde{D}^{(1)} \cap \widetilde{D}^{(2)} \cap \widetilde{D}^{(3)}$ is a $\mathbb{P}^{1}$-bundle over a $\mathbb{P}^{2}$-bundle over $\mathrm{Gr}^{\omega}(3,2 g)$.

(5) $\widetilde{D}^{(1)} \cap \widetilde{D}^{(2)}$ is the exceptional divisor of the blow-up $\mathbf{C C}(\mathcal{B}) \rightarrow \mathbb{P}\left(S^{2} \mathcal{B}\right)$. Hence $\widetilde{D}^{(1)} \cap \widetilde{D}^{(2)}$ is a $\mathbb{P}^{2}$-bundle over a $\mathbb{P}^{2}$-bundle over $\operatorname{Gr}^{\omega}(3,2 g)$.

We next investigate some rational curves that will be contracted. Define

$$
\begin{aligned}
\sigma & :=\text { class of a line in a } \mathbb{P}^{2} \text {-fiber of } \Phi_{B}^{\vee}, \\
\epsilon & :=\text { class of a line in a } \mathbb{P}^{2} \text {-fiber of } \Phi_{B}, \\
\gamma & :=\text { class of }\left\{\Phi_{B_{t}}^{-1}\left(\left[q_{t}\right]\right)\right\}_{t \in \Lambda}
\end{aligned}
$$

in $N_{1}\left(\widetilde{D}^{(1)}\right)$ (the group of numerical equivalence classes of 1-cycles) where $[A] \in \operatorname{Gr}^{\omega}(2,2 g)$, $q \in S^{2} A, A^{\perp}$ is the orthogonal complement of $A$ with respect to $\omega$, a line $\Lambda \subset \mathbb{P}\left(A^{\perp} / A\right)$ are fixed, $B_{t} \subset \mathbb{C}^{2 g}$ is the 3 -dimensional subspaces containing $A$ for $t \in \Lambda$ (that is, $\left.\left[B_{t}\right] \in \operatorname{Gr}^{\omega}(3,2 g)\right)$ and $q_{t} \in S^{2} B_{t}$ is the image of $q$ under the inclusion $S^{2} A \hookrightarrow S^{2} B_{t}$.

Let $\widehat{\sigma}=\iota_{*} \sigma, \widehat{\epsilon}=\iota_{*} \epsilon$ and $\widehat{\gamma}=\iota_{*} \gamma$ where $\iota$ is the inclusion $\widetilde{D}^{(1)} \hookrightarrow \mathbf{K}$. The following proposition tells us that $\mathbf{K}$ can be blown-down twice.

Proposition 7.5. (1) $\mathbb{R}^{+} \hat{\sigma}$ is a $\omega_{\mathbf{K}}$-negative extremal ray. The contraction $\mathbf{K}_{\sigma}$ of the ray $\mathbb{R}^{+} \hat{\sigma}$ is a smooth quasi-projective desingularization of $\mathbf{M}_{2}$. In fact, this is the contraction of the fiber direction of $\mathbb{P}\left(S^{2} \mathcal{A}\right) \rightarrow \operatorname{Gr}^{\omega}(2,2 g)$ in $\widetilde{D}^{(3)}$ and is also a blow-down map.

(2) For the image $\bar{\epsilon}$ of $\widehat{\epsilon}$ in $N_{1}\left(\mathbf{K}_{\sigma}\right), \mathbb{R}^{+} \bar{\epsilon}$ is $\omega_{\mathbf{K}_{\sigma}}$-negative extremal ray and its contraction $\mathbf{K}_{\epsilon}$ is a smooth quasi-projective desingularization of $\mathbf{M}_{2}$. This is the contraction of the fiber direction of $\mathbb{P}\left(S^{2} \mathcal{B}^{\vee}\right) \rightarrow \mathrm{Gr}^{\omega}(3,2 g)$ and is also a blow-down map. 
Proof. The proofs are identical to those of (3.0.2)-(3.0.4) in [18].

\subsection{A stratification of $\mathbf{S}$}

In this subsection, we provide a geometric description for the canonical subschemes of $\mathbf{S}$, which is useful for the proof of Theorem 6.1. The arguments are almost same as those of [1] except for the necessary modifications. Since $\mathbf{S}$ is a fine moduli scheme, we have the universal family $\left(\mathcal{E}_{\text {univ }}, \phi_{\text {univ }}, s_{\text {univ }}\right)$ of rank 4 and degree 0 on $X \times \mathbf{S}$. Consider the canonical family of specializations $B=p_{\mathbf{S} *} \mathcal{E} n d\left(\left(\mathcal{E}_{\text {univ }}, \phi_{\text {univ }}\right)\right)$ of $M(2)$ parametrized by $\mathbf{S}$ and the universal family of specializations $W$ of $M(2)$ parametrized by $\mathcal{A}_{2}$, where $p_{\mathbf{S}}: X \times \mathbf{S} \rightarrow \mathbf{S}$ is the projection onto the second component. Then $B$ and $W$ give generalized conic bundles $P$ on $\mathbf{S}$ and $Q$ on $\mathcal{A}_{2}$ respectively (see [1, Remark 3] for more details).

Proposition 7.6. [1, Proposition 2] $P=\pi_{\mathrm{sp}}^{*} Q$ for the morphism $\pi_{\mathrm{sp}}: \mathbf{S} \rightarrow \mathcal{A}_{2}$ given by $(E, \phi, s) \mapsto \operatorname{End}((E, \phi))$.

Proof. This is an immediate consequence of the definitions of $\pi_{s p}, B$ and $W$.

To define the canonical subschemes of $\mathcal{A}_{2}$, we need the concept of generalized conic bundle in the sense of [1] as follows.

Definition 7.7. [1, Definition 1] Let $Y$ be a variety. A generalized conic bundle $\mathcal{C}$ on $Y$ is defined by

(a) a vector bundle $V$ on $Y$ of rank 3 and

(b) a closed subscheme $\mathcal{C}$ of $\mathbb{P}(V)$ over $Y$, such that, given $y \in Y$, there exists a neighborhood $U$ of $y$, where $\mathcal{C} \cap p^{-1}(U)$ is defined by $q=0, q \in H^{0}\left(p^{-1}(U), H^{2}\right), H$ being the tautological line bundle for $p: \mathbb{P}(V) \rightarrow Y$, that is, $p_{*} H \cong V^{\vee}$.

Since $\mathcal{C}$ is an effective Cartier divisor by Definition 7.7, it corresponds to a section of a line bundle $L_{\mathcal{C}}$ on $\mathbb{P}(V)$. Since $L_{\mathcal{C}}$ and $H^{2}$ coincide locally over $Y$, it follows from the "see-saw" theorem (cf. Mumford's Abelian varieties) that there exists a line bundle $L$ on $Y$ such that $L_{\mathcal{C}}=H^{2} \otimes p^{*}(L)$. Since $p_{*} L_{\mathcal{C}}=p_{*}\left(H^{2}\right) \otimes L=S^{2}\left(V^{*}\right) \otimes L, q$ of the condition (b) in Definition 7.7 can be replaced by an element $q_{L} \in H^{0}\left(S^{2}\left(V^{*}\right) \otimes L\right)$, that is, a quadratic form $q_{L}: V \rightarrow L$. The discriminant $\Delta$ of $q_{L}$ is defined as a section of $L^{3} \otimes\left(\wedge^{3}\left(V^{\vee}\right)\right)^{2}$. The degeneracy locus of $\mathcal{C}$ is given by $\Delta=0$.

For $i=1,2,3$, set

$$
Y_{i}=\left\{y \in Y\left|q_{L}\right|_{\left.V\right|_{y}} \text { has rank } \leq 3-i\right\}
$$

Then we have $Y \supset Y_{1} \supset Y_{2} \supset Y_{3}$. We call $Y_{i}$ the canonical subschemes associated to the degenerate loci of the conic bundle $\mathcal{C}$ on $Y$. 
We have the canonical subschemes

$$
\mathbf{S} \supset \mathbf{S}_{1} \supset \mathbf{S}_{2} \supset \mathbf{S}_{3} \quad \text { and } \quad \mathcal{A}_{2} \supset\left(\mathcal{A}_{2}\right)_{1} \supset\left(\mathcal{A}_{2}\right)_{2} \supset\left(\mathcal{A}_{2}\right)_{3}
$$

associated to the degeneracy locus of $P$ and $Q$ respectively. Then, by Proposition 7.6, $\pi_{s p}$ maps $\mathbf{S}-\mathbf{S}_{2}$ into $\mathcal{A}_{2}-\left(\mathcal{A}_{2}\right)_{2}$ such that $\mathbf{S}_{1}-\mathbf{S}_{2} \rightarrow\left(\mathcal{A}_{2}\right)_{1}-\left(\mathcal{A}_{2}\right)_{2}$ and $\mathbf{S}-\mathbf{S}_{1} \rightarrow \mathcal{A}_{2}-\left(\mathcal{A}_{2}\right)_{1}$.

By Theorem 1 in [20], we have $\mathcal{A}_{2} \simeq \Phi \times \Lambda$, where $\Lambda$ is the 3 -dimensional affine space and $\Phi$ is the 6 -dimensional affine space whose points are identified with the set of quadratic forms on a fixed 3-dimensional vector space or the set of algebras of the form $C_{q}^{+}$, the even degree elements of the Clifford algebra associated to the quadratic form $q$. Hence we have

$$
\left(\mathcal{A}_{2}\right)_{i}=\{q \in \Phi \mid \operatorname{rank} q \leq 3-i\} \times \mathbb{C}^{3} \quad \text { for } i=1,2,3 \text {, }
$$

and

$$
\mathcal{A}_{2}-\left(\mathcal{A}_{2}\right)_{1}=\left\{q \in \Phi \mid C_{q}^{+} \simeq M(2)\right\} \times \mathbb{C}^{3}=\left\{y \in \mathcal{A}_{2} \mid W_{y} \simeq M(2)\right\} .
$$

Proposition 7.8. The subsets $\mathbf{S}-\mathbf{S}_{2}$ and $\mathbf{S}_{1}-\mathbf{S}_{2}$ of $\mathbf{S}$ are precisely $\mathbf{S}-\pi_{\mathbf{S}}^{-1}\left(\mathbb{Z}_{2}^{2 g}\right)$ and $\pi_{\mathbf{S}}^{-1}\left(T^{*} J / \mathbb{Z}_{2}-\mathbb{Z}_{2}^{2 g}\right)$ respectively. In particular, $\mathbf{S}-\mathbf{S}_{1}=\pi_{\mathbf{S}}^{-1}\left(\mathbf{M}_{2}^{s}\right)$.

Proof. The proof is similar to that of Proposition 3 in [1]. By the arguments before this proposition, it suffices to show that the subsets $\pi_{\mathbf{S}}^{-1}\left(\mathbf{M}_{2}^{s}\right)$ and $\pi_{\mathbf{S}}^{-1}\left(T^{*} J / \mathbb{Z}_{2}-\mathbb{Z}_{2}^{2 g}\right)$ of $\mathbf{S}$ are mapped by $\pi_{s p}$ into the subsets $\mathcal{A}_{2}-\left(\mathcal{A}_{2}\right)_{1}$ and $\left(\mathcal{A}_{2}\right)_{1}-\left(\mathcal{A}_{2}\right)_{2}$ of $\mathcal{A}_{2}$ respectively. We already know that $(E, \phi, s) \in \pi_{\mathbf{S}}^{-1}\left(\mathbf{M}_{2}^{s}\right)$ if and only if $\operatorname{End}((E, \phi))=M(2)$, which proves that $\pi_{\mathbf{S}}^{-1}\left(\mathbf{M}_{2}^{s}\right)$ maps to $\mathcal{A}_{2}-\left(\mathcal{A}_{2}\right)_{1}$.

Next, we claim that for $(E, \phi, s) \in \pi_{\mathbf{S}}^{-1}\left(T^{*} J / \mathbb{Z}_{2}-\mathbb{Z}_{2}^{2 g}\right)$, End $((E, \phi))$ has the same defining relations as that of $C_{q}^{+}$for a quadratic form $q$ of rank 2 on a 3-dimensional vector space. It follows from the definition of $\pi_{\mathbf{S}}$ that the endomorphism algebras of any two points in a fibre $\pi_{\mathbf{S}}^{-1}\left((L, \psi) \oplus\left(L^{-1},-\psi\right)\right)$ are isomorphic. So we can choose a point $(E, \phi, s) \in \pi_{\mathbf{S}}^{-1}\left((L, \psi) \oplus\left(L^{-1},-\psi\right)\right)$, where

$$
\begin{gathered}
(E, \phi)=\left(V, \phi_{V}\right) \oplus\left(W, \phi_{W}\right) \\
\left(V, \phi_{V}\right) \in H^{1}\left(L^{2}\right) \oplus H^{0}\left(L^{2} K_{X}\right), \quad\left(W, \phi_{W}\right) \in H^{1}\left(L^{-2}\right) \oplus H^{0}\left(L^{-2} K_{X}\right)
\end{gathered}
$$

and

$$
(L, \psi) \in T^{*} J-\mathbb{Z}_{2}^{2 g}
$$

that is,

$$
0 \rightarrow(L, \psi) \rightarrow\left(V, \phi_{V}\right) \rightarrow\left(L^{-1},-\psi\right) \rightarrow 0
$$

and

$$
0 \rightarrow\left(L^{-1},-\psi\right) \rightarrow\left(W, \phi_{W}\right) \rightarrow(L, \psi) \rightarrow 0
$$


It is obvious that this kinds of points belongs to $\pi_{\mathbf{S}}^{-1}\left(T^{*} J / \mathbb{Z}_{2}-\mathbb{Z}_{2}^{2 g}\right)$. By $(7.12)$ and $(7.13)$, we can see that $\operatorname{End}\left(\left(V, \phi_{V}\right) \oplus\left(W, \phi_{W}\right)\right)$ has four generators, which can be written as the following block matrices

$$
x=\left(\begin{array}{cc}
0 & 0 \\
\gamma_{2} & 0
\end{array}\right), \quad w=\left(\begin{array}{cc}
0 & \gamma_{1} \\
0 & 0
\end{array}\right), \quad u=\left(\begin{array}{cc}
I & 0 \\
0 & 0
\end{array}\right) \quad \text { and } \quad v=\left(\begin{array}{cc}
0 & 0 \\
0 & I
\end{array}\right)
$$

where $I$ is the $2 \times 2$ identity matrix and $\gamma_{1}, \gamma_{2}$ are the $2 \times 2$ matrices that come from identification of the rank 1 Higgs bundles in 7.12 and 7.13).

Here we need to show that $\operatorname{End}\left(\left(V, \phi_{V}\right)\right)=\mathbb{C} \cdot \operatorname{id}_{V}$ precisely. First of all we show that $\operatorname{End}(V)=\mathbb{C} \cdot \mathrm{id}_{V}$. Consider an arbitrary nontrivial morphism $\phi: V \rightarrow V$ in $\operatorname{End}(V)$. Let

$$
0 \longrightarrow L \stackrel{i}{\longrightarrow} V \stackrel{j}{\longrightarrow} L^{-1} \longrightarrow 0
$$

be the nonsplit extension to define $V$. Then the composition

$$
L \stackrel{i}{\longrightarrow} V \stackrel{\phi}{\longrightarrow} V \stackrel{j}{\longrightarrow} L^{-1}
$$

should be zero. If not, then this composition is an isomorphism, which is a contradiction. Then $\phi(L) \subset L$. Let $\psi:=\phi-\lambda \cdot \operatorname{id}_{V}$. Since $\left.\phi\right|_{L}=\lambda \cdot \operatorname{id}_{L}$ for some $\lambda \in \mathbb{C}^{\times},\left.\psi\right|_{L}=0$, that is, $\psi \circ i=0$, which implies that $\psi: V \rightarrow V$ factors through $V \stackrel{j}{\rightarrow} L^{-1}$ and there exists a unique morphism $\psi^{\prime}: L^{-1} \rightarrow V$ such that $\psi^{\prime} \circ j=\psi$. If the composition $L^{-1} \stackrel{\psi^{\prime}}{\rightarrow} V j L^{-1}$ is nonzero, then $j \circ \psi^{\prime}=\eta \cdot \mathrm{id}_{L^{-1}}$ for some $\eta \in \mathbb{C}^{\times}$. Then $\frac{1}{\eta} \psi^{\prime}: L^{-1} \rightarrow V$ is a splitting of the following nonplit extension

$$
0 \longrightarrow L \stackrel{i}{\longrightarrow} V \stackrel{j}{\longrightarrow} L^{-1} \longrightarrow 0
$$

which is a contradiction. So the composition $L^{-1} \stackrel{\psi^{\prime}}{\rightarrow} V \stackrel{j}{\rightarrow} L^{-1}$ is zero and then there exists a unique morphism $h: L^{-1} \rightarrow L$ such that $i \circ h=\psi^{\prime}$. Since $L \not L^{-1}, h=0$, that is, $\psi^{\prime}=0$. Hence $\psi=0$, that is, $\psi=\lambda \cdot \mathrm{id}_{V}$.

By Theorem 2.4. End $\left(\left(V, \psi_{V}\right)\right)=\mathbf{H}^{0} \mathbf{H o m}\left(\left(V, \phi_{V}\right),\left(V, \phi_{V}\right)\right)$. We know that

$$
\mathbf{H}^{0} \mathbf{H o m}\left(\left(V, \phi_{V}\right),\left(V, \phi_{V}\right)\right)=\operatorname{ker}\left(\operatorname{End}(V) \stackrel{\left[\cdot, \phi_{V}\right]}{\longrightarrow} \operatorname{Hom}\left(V, V \otimes K_{X}\right)\right) .
$$

Since $\operatorname{End}(V)=\mathbb{C} \cdot \operatorname{id}_{V}$, we have $\left[\cdot, \phi_{V}\right]=0$, that is, $\operatorname{End}\left(\left(V, \psi_{V}\right)\right)=\operatorname{End}(V)=\mathbb{C} \cdot \operatorname{id}_{V}$. Similarly, we can show that $\operatorname{End}\left(\left(W, \phi_{W}\right)\right)=\mathbb{C} \cdot \operatorname{id}_{W}$.

Coming back to the main proof, the defining relations of $\operatorname{End}\left(\left(V, \phi_{V}\right) \oplus\left(W, \phi_{W}\right)\right)$ can be written as

$$
\begin{gathered}
u^{2}=u, \quad v^{2}=v, \quad u v=0, \quad u+v=I, \quad w^{2}=x^{2}=w x=0, \quad u w=w, \\
w u=0, \quad u x=0, \quad x u=x, \quad v w=0, \quad w v=w, \quad v x=x, \quad x v=0 .
\end{gathered}
$$


For a quadratic form $q$ of rank 2 on a 3 -dimensional vector space over $\mathbb{C}$, we can see that $C_{q}^{+}$is a 4 -dimensional $\mathbb{C}$-algebra with

$$
C_{q}^{+}=\mathbb{C}+\mathbb{C} \alpha+\mathbb{C} \beta+\mathbb{C} \gamma \quad \text { such that } \alpha^{2}=-1, \alpha \beta=-\gamma, \alpha \gamma=\beta, \beta \alpha=\gamma, \gamma \alpha=-\beta \text {. }
$$

When we set $a=\frac{1}{2}(1+i \alpha), b=\frac{1}{2}(1-i \alpha), c=i \beta+\gamma$ and $d=i \beta-\gamma$ for $i=\sqrt{-1} \in \mathbb{C}, a$, $b, c, d$ are new generators of $C_{q}^{+}$with the following defining relations

$$
\begin{gathered}
a^{2}=a, \quad b^{2}=b, \quad a b=0, \quad a+b=1, \quad c^{2}=d^{2}=c d=0, \quad a c=c \\
c a=0, \quad a d=0, \quad d a=d, \quad b c=0, \quad c b=c, \quad b d=d, \quad d b=0 .
\end{gathered}
$$

Both (7.14) and 7.15 are the same.

Corollary 7.9. $\mathbf{S}_{1}-\mathbf{S}_{2} \cong \widetilde{D}^{(2)}-\left(\widetilde{D}^{(1)} \cup \widetilde{D}^{(3)}\right)$, that is, it is a free $\mathbb{Z}_{2}$-quotient of a $I_{2 g-3^{-}}$ bundle on $T^{*} J-\mathbb{Z}_{2}^{2 g}$, where $I_{2 g-3}$ is the incidence variety given by $I_{2 g-3}=\{(p, H) \in$ $\left.\mathbb{P}^{2 g-3} \times\left(\mathbb{P}^{2 g-3}\right)^{*} \mid p \in H\right\}$.

Proof. The proof is similar to that of [1, Corollary 1] except for using (7.14) and Proposition 3.4 .

We claim that if $(E, \phi, s) \in \mathbf{S}_{1}-\mathbf{S}_{2}=\pi_{\mathbf{S}}^{-1}\left(T^{*} J / \mathbb{Z}_{2}-\mathbb{Z}_{2}^{2 g}\right)$ and $\pi_{\mathbf{S}}((E, \phi, s))=(L, \psi) \oplus$ $\left(L^{-1},-\psi\right) \in T^{*} J / \mathbb{Z}_{2}-\mathbb{Z}_{2}^{2 g}$ then $(E, \phi)=\left(V, \phi_{V}\right) \oplus\left(W, \phi_{W}\right)$ for some extension classes

$$
\left[\left(V, \phi_{V}\right)\right] \in \mathbb{P} \mathbf{H}^{1} \mathbf{H o m}\left((L, \psi),\left(L^{-1},-\psi\right)\right)=\mathbb{P}\left(H^{1}\left(L^{-2}\right) \oplus H^{0}\left(L^{-2} K_{X}\right)\right)=\mathbb{P}^{2 g-3}
$$

and

$$
\left[\left(W, \phi_{W}\right)\right] \in \mathbb{P} \mathbf{H}^{1} \mathbf{H o m}\left(\left(L^{-1},-\psi\right),(L, \psi)\right)=\mathbb{P}\left(H^{1}\left(L^{2}\right) \oplus H^{0}\left(L^{2} K_{X}\right)\right)=\mathbb{P}^{2 g-3}
$$

Let $(E, \phi, s) \in \pi_{\mathbf{S}}^{-1}\left(T^{*} J / \mathbb{Z}_{2}-\mathbb{Z}_{2}^{2 g}\right)$ such that $\pi_{\mathbf{S}}((E, \phi, s))=(L, \psi) \oplus\left(L^{-1},-\psi\right) \in$ $T^{*} J / \mathbb{Z}_{2}-\mathbb{Z}_{2}^{2 g}$. Then $\operatorname{End}((E, \phi))$ has four generators $x, w, u, v$ with defining relations (7.14) as in the proof of Proposition 7.8. Let $\left(V, \phi_{V}\right)=\operatorname{ker} u$. Then $\left(V, \phi_{V}\right)$ is a Higgs subbundle of $(E, \phi)$ and we have an exact sequence

$$
0 \rightarrow\left(V, \phi_{V}\right) \rightarrow(E, \phi) \rightarrow\left(W, \phi_{W}\right) \rightarrow 0
$$

such that $\left(W, \phi_{W}\right)=\operatorname{ker} v$. Thus this exact sequence splits and then $(E, \phi)=\left(V, \phi_{V}\right) \oplus$ $\left(W, \phi_{W}\right)$.

By Proposition 3.4. $\left(V, \phi_{V}\right)$ and $\left(W, \phi_{W}\right)$ cannot be $(L, \psi) \oplus(L, \psi)$ or $\left(L^{-1},-\psi\right) \oplus$ $\left(L^{-1},-\psi\right)$ and we also rule out $\left(V, \phi_{V}\right)=(L, \psi) \oplus\left(L^{-1},-\psi\right)$ and $\left(W, \phi_{W}\right)=\left(L^{-1},-\psi\right) \oplus$ $(L, \psi)$. Hence

$\left[\left(V, \phi_{V}\right)\right] \in \mathbb{P} \mathbf{H}^{1} \mathbf{H o m}\left((L, \psi),\left(L^{-1},-\psi\right)\right)$ and $\left[\left(W, \phi_{W}\right)\right] \in \mathbb{P} \mathbf{H}^{1} \mathbf{H o m}\left(\left(L^{-1},-\psi\right),(L, \psi)\right)$. 
We next claim that if we choose $(c, d, e, f) \in\left[H^{0}\left(L^{-2} K_{X}\right) \oplus H^{0}\left(L^{2} K_{X}\right)\right] \oplus\left[H^{1}\left(L^{2}\right) \oplus\right.$ $\left.H^{1}\left(L^{-2}\right)\right]$ such that $\left[\left(V, \phi_{V}\right)\right]=[(f, c)]$ and $\left[\left(W, \phi_{W}\right)\right]=[(e, d)]$ then $(c, d, e, f) \in \bar{\Psi}^{-1}(0)$.

Assume that $(c, d, e, f) \notin \bar{\Psi}^{-1}(0)$. Let $(a, b) \in H^{0}\left(K_{X}\right) \oplus H^{1}\left(\mathcal{O}_{X}\right)$ be classes obtained from transition of $L$ and local Higgs field of $\psi$. Following the arguments of the proof of Lemma 7.3 , $\left(V, \phi_{V}\right)$ and $\left(W, \phi_{W}\right)$ are obtained by an elementary modification starting with a family $\left(\mathcal{F}, \varphi_{\mathcal{F}}\right)$ of semistable Higgs bundles over $X$ parametrized by

$$
\begin{gathered}
C=\left\{(a, b, z c, z d, z e, z f) \in H^{0}\left(K_{X}\right) \oplus H^{1}\left(\mathcal{O}_{X}\right) \oplus\left[H^{0}\left(L^{-2} K_{X}\right) \oplus H^{0}\left(L^{2} K_{X}\right)\right]\right. \\
\left.\oplus\left[H^{1}\left(L^{2}\right) \oplus H^{1}\left(L^{-2}\right)\right] \mid z \in \mathbb{C}\right\}
\end{gathered}
$$

Precisely, $\left(V, \phi_{V}\right)=\left.\left(\mathcal{F}^{\prime}, \varphi_{\mathcal{F}^{\prime}}\right)\right|_{z=0}$ and $\left(W, \phi_{W}\right)=\left.\left(\mathcal{F}^{\prime \prime}, \varphi_{\mathcal{F}^{\prime \prime}}\right)\right|_{z=0}$, where $\left(\mathcal{F}^{\prime}, \varphi_{\mathcal{F}^{\prime}}\right)$ is the kernel of $\left.\left(\mathcal{F}, \varphi_{\mathcal{F}}\right) \rightarrow\left(\mathcal{F}, \varphi_{\mathcal{F}}\right)\right|_{z=0} \cong\left(L^{-1},-\psi\right) \oplus\left(L^{-1},-\psi\right) \rightarrow(L, \psi)$ and $\left(\mathcal{F}^{\prime \prime}, \varphi_{\mathcal{F}^{\prime \prime}}\right)$ is the kernel of $\left.\left(\mathcal{F}, \varphi_{\mathcal{F}}\right) \rightarrow\left(\mathcal{F}, \varphi_{\mathcal{F}}\right)\right|_{z=0} \cong\left(L^{-1},-\psi\right) \oplus\left(L^{-1},-\psi\right) \rightarrow\left(L^{-1},-\psi\right)$.

But since $(c, d, e, f) \notin \bar{\Psi}^{-1}(0),(z c, z d, z e, z f) \notin \bar{\Psi}^{-1}(0)$ for all $z \in \mathbb{C}$ and then $\left.\left(\mathcal{F}, \varphi_{\mathcal{F}}\right)\right|_{X \times(a, b, z c, z d, z e, z f)}$ is not a Higgs bundle for all $z \in \mathbb{C}$. Then $\left(V, \phi_{V}\right)$ and $\left(W, \phi_{W}\right)$ are not Higgs bundles. Indeed, if $\left(V, \phi_{V}\right)$ and $\left(W, \phi_{W}\right)$ are Higgs bundles and if we consider the following elementary modifications

$$
0 \rightarrow\left(\mathcal{G}^{\prime}, \varphi_{\mathcal{G}^{\prime}}\right) \rightarrow\left(\mathcal{F}^{\prime}, \varphi_{\mathcal{F}^{\prime}}\right) \rightarrow\left(L^{-1},-\psi\right) \rightarrow 0
$$

and

$$
0 \rightarrow\left(\mathcal{G}^{\prime \prime}, \varphi_{\mathcal{G}^{\prime \prime}}\right) \rightarrow\left(\mathcal{F}^{\prime \prime}, \varphi_{\mathcal{F}^{\prime \prime}}\right) \rightarrow(L, \psi) \rightarrow 0
$$

then $\left(\mathcal{G}^{\prime}, \varphi_{\mathcal{G}^{\prime}}\right) \cong\left(\mathcal{G}^{\prime \prime}, \varphi_{\mathcal{G}^{\prime \prime}}\right) \cong\left(\mathcal{F} \otimes \mathcal{O}_{X \times C}(-D), \varphi_{\mathcal{F} \otimes \mathcal{O}_{X \times C}(-D)}\right)$, where $D=\{(x,(a, b, z c, z d$, $z e, z f)) \in X \times C \mid z=0\}$. Since $\left.\left(\mathcal{F} \otimes \mathcal{O}_{X \times C}(-D), \varphi_{\mathcal{F} \otimes \mathcal{O}_{X \times C}(-D)}\right)\right|_{z=0}$ is a Higgs bundle and $\mathcal{O}_{X \times C}(-D)$ is a line bundle, $\left(V, \phi_{V}\right)$ and $\left(W, \phi_{W}\right)$ are Higgs bundles. Thus we get a contradiction. Hence $(c, d, e, f) \in \bar{\Psi}^{-1}(0)$.

\subsection{Factorization of $\rho$}

In this subsection, we show that $\rho$ factors through $\mathbf{K}_{\epsilon}$. Let $\mathbf{D}_{1}, \mathbf{D}_{2}$ and $\mathbf{D}_{3}$ be three exceptional divisors on $\mathbf{K}$ coming from the three blow-ups. Consider the point $0=$ $\left[\left(\mathcal{O}_{X}, 0\right) \oplus\left(\mathcal{O}_{X}, 0\right)\right] \in \mathbb{Z}_{2}^{2 g}$. Let $\widetilde{D}^{(1)}, \widetilde{D}^{(2)}$ and $\widetilde{D}^{(3)}$ be the restrictions of $\mathbf{D}_{1}$, $\mathbf{D}_{2}$ and $\mathbf{D}_{3}$ to a neighborhood of the preimage of 0 in $\mathbf{K}$ respectively. We need the following two lemmas for the proof.

Lemma 7.10. (1) The isomorphism classes of Higgs bundles given by (7.5) and (7.6) are independent of the choice of $(a, d),(b, e)$.

(2) The isomorphism classes of Higgs bundles given by (7.8) and (7.9) are independent of the choice of $(g, h),(b, e),(c, f)$. 
Proof. The proofs of (1) and (2) are identical. We prove only (1). Let $\left\{V_{i}\right\}_{i}$ be an open cover of $X$ and let $(E, \phi)$ and $\left(E^{\prime}, \phi^{\prime}\right)$ be Higgs bundles given by 7.5 on $V_{i} \cap V_{j}$ and 7.6 on $V_{i}$. Assume that an isomorphism between $(E, \phi)$ and $\left(E^{\prime}, \phi^{\prime}\right)$ is given by invertible matrices

$$
\left(\begin{array}{cccc}
\eta_{i, 11} & \eta_{i, 12} & \eta_{i, 13} & \eta_{i, 14} \\
\eta_{i, 21} & \eta_{i, 22} & \eta_{i, 23} & \eta_{i, 24} \\
\eta_{i, 31} & \eta_{i, 32} & \eta_{i, 33} & \eta_{i, 34} \\
\eta_{i, 41} & \eta_{i, 42} & \eta_{i, 43} & \eta_{i, 44}
\end{array}\right)
$$

on $V_{i}$. Then

$$
\begin{aligned}
& =\left(\begin{array}{llll}
\eta_{j, 11} & \eta_{j, 12} & \eta_{j, 13} & \eta_{j, 14} \\
\eta_{j, 21} & \eta_{j, 22} & \eta_{j, 23} & \eta_{j, 24} \\
\eta_{j, 31} & \eta_{j, 32} & \eta_{j, 33} & \eta_{j, 34} \\
\eta_{j, 41} & \eta_{j, 42} & \eta_{j, 43} & \eta_{j, 44}
\end{array}\right)\left(\begin{array}{cccc}
1 & 0 & 0 & 0 \\
0 & 1 & 0 & 0 \\
0 & 0 & 1 & 0 \\
-\left.2 \alpha_{i j}\right|_{z=0} & -\left.\beta_{i j}\right|_{z=0} & \left.\gamma_{i j}\right|_{z=0} & 1
\end{array}\right) \\
& =\left(\begin{array}{crrr}
1 & 0 & 0 & 0 \\
0 & 1 & 0 & 0 \\
0 & 0 & 1 & 0 \\
-\left.2 \alpha_{i j}^{\prime}\right|_{z=0} & -\left.\beta_{i j}^{\prime}\right|_{z=0} & \left.\gamma_{i j}^{\prime}\right|_{z=0} & 1
\end{array}\right)\left(\begin{array}{llll}
\eta_{i, 11} & \eta_{i, 12} & \eta_{i, 13} & \eta_{i, 14} \\
\eta_{i, 21} & \eta_{i, 22} & \eta_{i, 23} & \eta_{i, 24} \\
\eta_{i, 31} & \eta_{i, 32} & \eta_{i, 33} & \eta_{i, 34} \\
\eta_{i, 41} & \eta_{i, 42} & \eta_{i, 43} & \eta_{i, 44}
\end{array}\right)
\end{aligned}
$$

and

$$
\begin{aligned}
& \left(\begin{array}{cccc}
\eta_{i, 11} & \eta_{i, 12} & \eta_{i, 13} & \eta_{i, 14} \\
\eta_{i, 21} & \eta_{i, 22} & \eta_{i, 23} & \eta_{i, 24} \\
\eta_{i, 31} & \eta_{i, 32} & \eta_{i, 33} & \eta_{i, 34} \\
\eta_{i, 41} & \eta_{i, 42} & \eta_{i, 43} & \eta_{i, 44}
\end{array}\right)\left(\begin{array}{cccc}
0 & 0 & 0 & 0 \\
0 & 0 & 0 & 0 \\
0 & 0 & 0 & 0 \\
-\left.2 p_{i}\right|_{z=0} & -\left.q_{i}\right|_{z=0} & \left.r_{i}\right|_{z=0} & 0
\end{array}\right) \\
= & \left(\begin{array}{rrrr}
0 & 0 & 0 & 0 \\
0 & 0 & 0 & 0 \\
0 & 0 & 0 & 0 \\
-\left.2 p_{i}^{\prime}\right|_{z=0} & -\left.q_{i}^{\prime}\right|_{z=0} & \left.r_{i}^{\prime}\right|_{z=0} & 0
\end{array}\right)\left(\begin{array}{llll}
\eta_{i, 11} & \eta_{i, 12} & \eta_{i, 13} & \eta_{i, 14} \\
\eta_{i, 21} & \eta_{i, 22} & \eta_{i, 23} & \eta_{i, 24} \\
\eta_{i, 31} & \eta_{i, 32} & \eta_{i, 33} & \eta_{i, 34} \\
\eta_{i, 41} & \eta_{i, 42} & \eta_{i, 43} & \eta_{i, 44}
\end{array}\right) .
\end{aligned}
$$

Equating each rows, we have

$$
\begin{gathered}
\eta_{i, 11}=\eta_{j, 11}=\mathrm{constant}, \eta_{i, 12}=\eta_{j, 12}=\mathrm{constant}, \eta_{i, 13}=\eta_{j, 13}=\mathrm{constant}, \eta_{i, 14}=\eta_{j, 14}=0, \\
\eta_{i, 21}=\eta_{j, 21}=\mathrm{constant}, \eta_{i, 22}=\eta_{j, 22}=\mathrm{constant}, \eta_{i, 23}=\eta_{j, 23}=\mathrm{constant}, \eta_{i, 24}=\eta_{j, 24}=0, \\
\eta_{i, 31}=\eta_{j, 31}=\mathrm{constant}, \eta_{i, 32}=\eta_{j, 32}=\mathrm{constant}, \eta_{i, 33}=\eta_{j, 33}=\mathrm{constant}, \eta_{i, 34}=\eta_{j, 34}=0, \\
\eta_{i, 44}=\eta_{j, 44}=\mathrm{constant} \neq 0,
\end{gathered}
$$


and

$$
\begin{aligned}
-2 \eta_{i, 44}(a, d) & =-2 \eta_{i, 11}\left(a^{\prime}, d^{\prime}\right)-\eta_{i, 21}\left(b^{\prime}, e^{\prime}\right)+\eta_{i, 31}\left(c^{\prime}, f^{\prime}\right), \\
-\eta_{i, 44}(b, e) & =-2 \eta_{i, 12}\left(a^{\prime}, d^{\prime}\right)-\eta_{i, 22}\left(b^{\prime}, e^{\prime}\right)+\eta_{i, 32}\left(c^{\prime}, f^{\prime}\right), \\
\eta_{i, 44}(c, f) & =-2 \eta_{i, 13}\left(a^{\prime}, d^{\prime}\right)-\eta_{i, 23}\left(b^{\prime}, e^{\prime}\right)+\eta_{i, 33}\left(c^{\prime}, f^{\prime}\right),
\end{aligned}
$$

where

$$
\begin{gathered}
\left(\left\{\left.\alpha_{i j}\right|_{z=0}\right\},\left\{\left.p_{i}\right|_{z=0}\right\}\right), \quad\left(\left\{\left.\alpha_{i j}^{\prime}\right|_{z=0}\right\},\left\{\left.p_{i}^{\prime}\right|_{z=0}\right\}\right), \quad\left(\left\{\left.\beta_{i j}\right|_{z=0}\right\},\left\{\left.q_{i}\right|_{z=0}\right\}\right), \\
\left(\left\{\left.\beta_{i j}^{\prime}\right|_{z=0}\right\},\left\{\left.q_{i}^{\prime}\right|_{z=0}\right\}\right), \quad\left(\left\{\left.\gamma_{i j}\right|_{z=0}\right\},\left\{\left.r_{i}\right|_{z=0}\right\}\right) \quad \text { and } \quad\left(\left\{\left.\gamma_{i j}^{\prime}\right|_{z=0}\right\},\left\{\left.r_{i}^{\prime}\right|_{z=0}\right\}\right)
\end{gathered}
$$

represent classes $(a, d),\left(a^{\prime}, d^{\prime}\right),(b, e),\left(b^{\prime}, e^{\prime}\right),(c, f)$ and $\left(c^{\prime}, f^{\prime}\right)$ in $H^{1}\left(\mathcal{O}_{X}\right) \oplus H^{0}\left(K_{X}\right)$ respectively.

Since

$$
\left(\begin{array}{ccc}
-2 \eta_{i, 11} & -\eta_{i, 21} & \eta_{i, 31} \\
-2 \eta_{i, 12} & -\eta_{i, 22} & \eta_{i, 32} \\
-2 \eta_{i, 13} & -\eta_{i, 23} & \eta_{i, 33}
\end{array}\right)=\left(\begin{array}{ccc}
\eta_{i, 11} & \eta_{i, 21} & \eta_{i, 31} \\
\eta_{i, 12} & \eta_{i, 22} & \eta_{i, 32} \\
\eta_{i, 13} & \eta_{i, 23} & \eta_{i, 33}
\end{array}\right)\left(\begin{array}{ccc}
-2 & 0 & 0 \\
0 & -1 & 0 \\
0 & 0 & 1
\end{array}\right)
$$

is invertible, $\operatorname{Span}\{(a, d),(b, e),(c, f)\}=\operatorname{Span}\left\{\left(a^{\prime}, d^{\prime}\right),\left(b^{\prime}, e^{\prime}\right),\left(c^{\prime}, f^{\prime}\right)\right\}$.

Lemma 7.11. The isomorphism classes of Higgs bundles given by (7.10) and (7.11) are independent of the choice of $(b, e),(c, f)$.

Proof. Let $\left\{V_{i}\right\}_{i}$ be an open cover of $X$ and let $(E, \phi)$ and $\left(E^{\prime}, \phi^{\prime}\right)$ be Higgs bundles given by 7.10 on $V_{i} \cap V_{j}$ and 7.11 on $V_{i}$. Assume that an isomorphism between $(E, \phi)$ and $\left(E^{\prime}, \phi^{\prime}\right)$ is given by invertible matrices

$$
\left(\begin{array}{cccc}
\eta_{i, 11} & \eta_{i, 12} & \eta_{i, 13} & \eta_{i, 14} \\
\eta_{i, 21} & \eta_{i, 22} & \eta_{i, 23} & \eta_{i, 24} \\
\eta_{i, 31} & \eta_{i, 32} & \eta_{i, 33} & \eta_{i, 34} \\
\eta_{i, 41} & \eta_{i, 42} & \eta_{i, 43} & \eta_{i, 44}
\end{array}\right)
$$

on $V_{i}$. Then

$$
\left(\begin{array}{cccc}
\eta_{j, 11} & \eta_{j, 12} & \eta_{j, 13} & \eta_{j, 14} \\
\eta_{j, 21} & \eta_{j, 22} & \eta_{j, 23} & \eta_{j, 24} \\
\eta_{j, 31} & \eta_{j, 32} & \eta_{j, 33} & \eta_{j, 34} \\
\eta_{j, 41} & \eta_{j, 42} & \eta_{j, 43} & \eta_{j, 44}
\end{array}\right)\left(\begin{array}{cccc}
1 & 0 & 0 & 0 \\
\left.\gamma_{i j}\right|_{z=0} & 1 & 0 & 0 \\
\left.\beta_{i j}\right|_{z=0} & 0 & 1 & 0 \\
-\left.2 \delta_{i j}\right|_{z=0} & -\left.\beta_{i j}\right|_{z=0} & \left.\gamma_{i j}\right|_{z=0} & 1
\end{array}\right)
$$




$$
=\left(\begin{array}{cccc}
1 & 0 & 0 & 0 \\
\left.\gamma_{i j}^{\prime}\right|_{z=0} & 1 & 0 & 0 \\
\left.\beta_{i j}^{\prime}\right|_{z=0} & 0 & 1 & 0 \\
-\left.2 \delta_{i j}^{\prime}\right|_{z=0} & -\left.\beta_{i j}^{\prime}\right|_{z=0} & \left.\gamma_{i j}^{\prime}\right|_{z=0} & 1
\end{array}\right)\left(\begin{array}{cccc}
\eta_{i, 11} & \eta_{i, 12} & \eta_{i, 13} & \eta_{i, 14} \\
\eta_{i, 21} & \eta_{i, 22} & \eta_{i, 23} & \eta_{i, 24} \\
\eta_{i, 31} & \eta_{i, 32} & \eta_{i, 33} & \eta_{i, 34} \\
\eta_{i, 41} & \eta_{i, 42} & \eta_{i, 43} & \eta_{i, 44}
\end{array}\right)
$$

and

$$
\begin{aligned}
& \left(\begin{array}{cccc}
\eta_{i, 11} & \eta_{i, 12} & \eta_{i, 13} & \eta_{i, 14} \\
\eta_{i, 21} & \eta_{i, 22} & \eta_{i, 23} & \eta_{i, 24} \\
\eta_{i, 31} & \eta_{i, 32} & \eta_{i, 33} & \eta_{i, 34} \\
\eta_{i, 41} & \eta_{i, 42} & \eta_{i, 43} & \eta_{i, 44}
\end{array}\right)\left(\begin{array}{cccc}
0 & 0 & 0 & 0 \\
\left.r_{i}\right|_{z=0} & 0 & 0 & 0 \\
\left.q_{i}\right|_{z=0} & 0 & 0 & 0 \\
-\left.2 s_{i}\right|_{z=0} & -\left.q_{i}\right|_{z=0} & \left.r_{i}\right|_{z=0} & 0
\end{array}\right) \\
= & \left(\begin{array}{cccc}
0 & 0 & 0 & 0 \\
\left.r_{i}^{\prime}\right|_{z=0} & 0 & 0 & 0 \\
\left.q_{i}^{\prime}\right|_{z=0} & 0 & 0 & 0 \\
-\left.2 s_{i}^{\prime}\right|_{z=0} & -\left.q_{i}^{\prime}\right|_{z=0} & \left.r_{i}^{\prime}\right|_{z=0} & 0
\end{array}\right)\left(\begin{array}{llll}
\eta_{i, 11} & \eta_{i, 12} & \eta_{i, 13} & \eta_{i, 14} \\
\eta_{i, 21} & \eta_{i, 22} & \eta_{i, 23} & \eta_{i, 24} \\
\eta_{i, 31} & \eta_{i, 32} & \eta_{i, 33} & \eta_{i, 34} \\
\eta_{i, 41} & \eta_{i, 42} & \eta_{i, 43} & \eta_{i, 44}
\end{array}\right) .
\end{aligned}
$$

Equating each rows, we have

$$
\begin{gathered}
\eta_{i, 11}=\eta_{j, 11}=\mathrm{constant}, \quad \eta_{i, 12}=\eta_{j, 12}=0, \quad \eta_{i, 13}=\eta_{j, 13}=0, \quad \eta_{i, 14}=\eta_{j, 14}=0, \\
\eta_{i, 22}=\eta_{j, 22}=\mathrm{constant}, \quad \eta_{i, 23}=\eta_{j, 23}=\mathrm{constant}, \quad \eta_{i, 24}=\eta_{j, 24}=0, \\
\eta_{i, 32}=\eta_{j, 32}=\mathrm{constant}, \quad \eta_{i, 33}=\eta_{j, 33}=\mathrm{constant}, \quad \eta_{i, 34}=\eta_{j, 34}=0, \\
\eta_{i, 44}=\eta_{j, 44}=\mathrm{constant},
\end{gathered}
$$

and

$$
\begin{aligned}
-\eta_{i, 22}(c, f)-\eta_{i, 23}(b, e)+\eta_{i, 11}\left(c^{\prime}, f^{\prime}\right) & =0, \\
-\eta_{i, 32}(c, f)-\eta_{i, 33}(b, e)+\eta_{i, 11}\left(b^{\prime}, e^{\prime}\right) & =0, \\
-\eta_{i, 22}\left(b^{\prime}, e^{\prime}\right)+\eta_{i, 32}\left(c^{\prime}, f^{\prime}\right)+\eta_{i, 44}(b, e) & =0, \\
-\eta_{i, 23}\left(b^{\prime}, e^{\prime}\right)+\eta_{i, 33}\left(c^{\prime}, f^{\prime}\right)-\eta_{i, 44}(c, f) & =0,
\end{aligned}
$$

where

$$
\begin{gathered}
\left(\left\{\left.\delta_{i j}\right|_{z=0}\right\},\left\{\left.s_{i}\right|_{z=0}\right\}\right), \quad\left(\left\{\left.\delta_{i j}^{\prime}\right|_{z=0}\right\},\left\{\left.s_{i}^{\prime}\right|_{z=0}\right\}\right), \quad\left(\left\{\left.\beta_{i j}\right|_{z=0}\right\},\left\{\left.q_{i}\right|_{z=0}\right\}\right), \\
\left(\left\{\left.\beta_{i j}^{\prime}\right|_{z=0}\right\},\left\{\left.q_{i}^{\prime}\right|_{z=0}\right\}\right), \quad\left(\left\{\left.\gamma_{i j}\right|_{z=0}\right\},\left\{\left.r_{i}\right|_{z=0}\right\}\right) \quad \text { and } \quad\left(\left\{\left.\gamma_{i j}^{\prime}\right|_{z=0}\right\},\left\{\left.r_{i}^{\prime}\right|_{z=0}\right\}\right)
\end{gathered}
$$

represent classes $(g, h),\left(g^{\prime}, h^{\prime}\right),(b, e),\left(b^{\prime}, e^{\prime}\right),(c, f)$ and $\left(c^{\prime}, f^{\prime}\right)$ in $H^{1}\left(\mathcal{O}_{X}\right) \oplus H^{0}\left(K_{X}\right)$ respectively. Hence

$$
\operatorname{Span}\{(b, e),(c, f)\}=\operatorname{Span}\left\{\left(b^{\prime}, e^{\prime}\right),\left(c^{\prime}, f^{\prime}\right)\right\}
$$


Proposition 7.12. $\rho$ factors through $\mathbf{K}_{\epsilon}$.

Proof. Consider the first contraction $f_{\sigma}: \mathbf{K} \rightarrow \mathbf{K}_{\sigma}$. We show that there is a continuous map $\rho_{\sigma}: \mathbf{K}_{\sigma} \rightarrow \mathbf{S}$ such that $\rho_{\sigma} \circ f_{\sigma}=\rho$. It is enough to show that $\rho$ is constant on the fibers of $f_{\sigma}$ by Riemann's extension theorem. By Propositions 7.4 and 7.5, $f_{\sigma}$ is indeed the contraction of the fibers $\mathbb{P}^{2}$ of $\mathbb{P}\left(S^{2} \mathcal{A}\right) \rightarrow \operatorname{Gr}^{\omega}(2,2 g)$ in $\widetilde{D}^{(3)}$ which forgets the choice of $(b, e),(c, f)$ in the 2-dimensional subspace of $H^{1}\left(\mathcal{O}_{X}\right) \oplus H^{0}\left(K_{X}\right)$ spanned by $(b, e),(c, f)$. We have only to check that the isomorphism classes of the Higgs bundles given by (7.8), (7.9), 7.10 and 7.11) depend not on the particular choice of $(b, e),(c, f)$ but only on the points in $\mathbb{P}^{2 g-4}$-bundle over $\operatorname{Gr}^{\omega}(2,2 g)$. By Lemma 7.11, the isomorphism classes of Higgs bundles given by (7.10) and (7.11) are independent of the choice of $(b, e),(c, f)$. On the other hand, it follows from Lemma $7.10(2)$ that the isomorphism classes of the Higgs bundles given by (7.8) and (7.9) are independent of the choice of $(g, h),(b, e),(c, f)$. Thus, there exists a morphism $\rho_{\sigma}: \mathbf{K}_{\sigma} \rightarrow \mathbf{S}$ such that $\rho_{\sigma} \circ f_{\sigma}=\rho$.

We next show that $\rho_{\sigma}$ factors through $\mathbf{K}_{\epsilon}$. By Proposition 7.5, the morphism $f_{\epsilon}: \mathbf{K}_{\sigma} \rightarrow$ $\mathbf{K}_{\epsilon}$ is the contraction of the fiber $\mathbb{P}^{5}$ of a $\mathbb{P}^{5}$-bundle over $\operatorname{Gr}^{\omega}(3,2 g)$ and general points of a fiber give rise to a rank 4 Higgs bundle given by (7.5) and (7.6). By Lemma 7.10(1), the isomorphism classes of the Higgs bundles given by $(7.5)$ and $(7.6)$ depend only on the 3dimensional subspace spanned by $(a, d),(b, e),(c, f)$. Thus $\rho_{\sigma}$ is constant along the fibers of $f_{\epsilon}$. Applying Riemann's extension theorem again, we obtain a morphism $\rho_{\epsilon}: \mathbf{K}_{\epsilon} \rightarrow \mathbf{S}$ such that $\rho_{\epsilon} \circ f=\rho$.

\subsection{A proof of Theorem 6.1}

We precisely refer to the original version of Zariski's main theorem.

Proposition 7.13. (Main theorem of [25]) If $W$ is an irreducible fundamental variety on $V$ of a birational correspondence $T$ between $V$ and $V^{\prime}$ and if $T$ has no fundamental elements on $V^{\prime}$, then-under the assumption that $V$ is locally normal at $W$-each irreducible component of the transform $T[W]$ is of higher dimension than $W$.

By Proposition 7.8 and Corollary $7.9, \rho\left(\widetilde{D}^{(2)}-\widetilde{D}^{(1)} \cup \widetilde{D}^{(3)}\right)=\mathbf{S}_{1}-\mathbf{S}_{2}$ is a smooth divisor of $\mathbf{S}-\rho\left(\widetilde{D}^{(1)} \cup \widetilde{D}^{(3)}\right)=\mathbf{S}-\mathbf{S}_{2}$ lying over $T^{*} J / \mathbb{Z}_{2}-\mathbb{Z}_{2}^{2 g}$. Then we have a morphism $g$ from $\mathbf{S}-\rho\left(\widetilde{D}^{(1)} \cup \widetilde{D}^{(3)}\right)$ to the blow-up of $\mathbf{M}_{2}-\mathbb{Z}_{2}^{2 g}$ along $T^{*} J / \mathbb{Z}_{2}-\mathbb{Z}_{2}^{2 g}$ which is isomorphic to $\mathbf{K}-\widetilde{D}^{(1)} \cup \widetilde{D}^{(3)}=\mathbf{K}_{\epsilon}-f\left(\widetilde{D}^{(1)} \cup \widetilde{D}^{(3)}\right)$. Thus, $\rho_{\epsilon}$ mentioned in the proof of Proposition 7.12 and $g$ are isomorphisms in codimension one. Note that $f\left(\widetilde{D}^{(1)} \cup \widetilde{D}^{(3)}\right)$ has codimension 3 in $\mathbf{K}_{\epsilon}$.

Assume that $\mathbf{K}_{\epsilon}$ is not isomorphic to $\mathbf{S}$. Since $\mathbf{S}$ is smooth and $\mathbf{S}_{2}$ is the fundamental variety on $\mathbf{S}$ of $\rho_{\epsilon}$, it follows from Proposition 7.13 that $\mathbf{S}_{2}$ has codimension greater than 3 . Further, since $\mathbf{K}_{\epsilon}$ is smooth, $f\left(\widetilde{D}^{(1)} \cup \widetilde{D}^{(3)}\right)$ is the fundamental variety on $\mathbf{K}_{\epsilon}$ of $g$, it follows 
from Proposition 7.13 that $\mathbf{S}_{2}$ has codimension less than 3. Hence to avoid a contradiction, $\mathbf{K}_{\epsilon}$ should be isomorphic to $\mathbf{S}$.

\section{Acknowledgments}

First of all I wish to thank my supervisor Young-Hoon Kiem for suggesting problem, and for his help and encouragement. I am grateful to Conjeevaram Srirangachari Seshadri for his inspiring papers [20,21]. I also thank Insong Choe for useful comments.

\section{References}

[1] V. Balaji, Cohomology of certain moduli spaces of vector bundles, Proc. Indian Acad. Sci. Math. Sci. 98 (1988), no. 1, 1-24.

[2] A. Beauville, M. S. Narasimhan and S. Ramanan, Spectral curves and the generalised theta divisor, J. Reine Angew. Math. 398 (1989), 169-179.

[3] I. Biswas and S. Ramanan, An infinitesimal study of the moduli of Hitchin pairs, J. London Math. Soc. (2) 49 (1994), no. 2, 219-231.

[4] S. B. Bradlow, O. García-Prada and P. B. Gothen, Moduli spaces of holomorphic triples over compact Riemann surfaces, Math. Ann. 328 (2004), no. 1-2, 299-351.

[5] W. M. Goldman and J. J. Millson, The deformation theory of representations of fundamental groups of compact Kähler manifolds, Inst. Hautes Études Sci. Publ. Math. 67 (1988), 43-96.

[6] R. Hartshorne, Deformation Theory, Graduate Texts in Mathematics 257, Springer, New York, 2010.

[7] T. Hausel, Compactification of moduli of Higgs bundles, J. Reine Angew. Math. 503 (1998), 169-192.

[8] T. Hausel and C. Pauly, Prym varieties of spectral covers, Geom. Topol. 16 (2012), no. 3, 1609-1638.

[9] T. Hausel and M. Thaddeus, Generators for the cohomology ring of the moduli space of rank 2 Higgs bundles, Proc. London Math. Soc. (3) 88 (2004), no. 3, 632-658.

[10] N. J. Hitchin, The self-duality equations on a Riemann surface, Proc. London Math. Soc. (3) 55 (1987), no. 1, 59-126. 
[11] D. Huybrechts and M. Lehn, The Geometry of Moduli Spaces of Sheaves, Aspects of Mathematics E31, Friedr. Vieweg \& Sohn, Braunschweig, 1997.

[12] Y.-H. Kiem and J. Li, Desingularizations of the moduli space of rank 2 bundles over a curve, Math. Ann. 330 (2004), no. 3, 491-518.

[13] Y.-H. Kiem and S.-B. Yoo, The stringy E-function of the moduli space of Higgs bundles with trivial determinant, Math. Nachr. 281 (2008), no. 6, 817-838.

[14] F. C. Kirwan, Partial desingularisations of quotients of nonsingular varieties and their Betti numbers, Ann. of Math. (2) 122 (1985), no. 1, 41-85.

[15] M. Mehta, Birational equivalence of Higgs moduli, Internat. J. Math. 16 (2005), no. 4, $365-386$.

[16] D. Mumford, Algebraic Geometry I: Complex Projective Varieties, Springer, Berlin, 1976.

[17] N. Nitsure, Moduli space of semistable pairs on a curve, Proc. London Math. Soc. (3) 62 (1991), no. 2, 275-300.

[18] K. G. O'Grady, Desingularized moduli spaces of sheaves on a K3, I, arXiv:alggeom/9708009.

[19] Desingularized moduli spaces of sheaves on a K3, J. Reine Angew. Math. 512 (1999), 49-117.

[20] C. S. Seshadri, Desingularisation of the moduli varieties of vector bundles on curves, in: Proceedings of the International Symposium on Algebraic Geometry (Kyoto Univ., Kyoto, 1977), 155-184, Kinokuniya Book Store, Tokyo, 1978.

[21] _ Fibrés vectoriels sur les courbes algébriques, Astérisque 96, Société Mathématique de France, Paris, 1982.

[22] C. T. Simpson, Moduli of representations of the fundamental group of a smooth projective variety I and II, Inst. Hautes Études Sci. Publ. Math. 79 (1994), 47-129 and 80 (1994), 5-79.

[23] K. Yokogawa, Compactification of moduli of parabolic sheaves and moduli of parabolic Higgs sheaves, J. Math. Kyoto Univ. 33 (1993), no. 2, 451-504.

[24] _ Infinitesimal deformation of parabolic Higgs sheaves, Internat. J. Math. 6 (1995), no. 1, 125-148. 
[25] O. Zariski, Foundations of a general theory of birational correspondences, Trans. Amer. Math. Soc. 53 (1943), 490-542.

Sang-Bum Yoo

Department of Mathematics Education, Gongju National University of Education, Gongju-si, Chungcheongnam-do, 32553, South Korea

E-mail address: sbyoo@gjue.ac.kr 